Experimental determination of drag coefficients in low-density polyurethane foam

M. L. Adams

April 20, 2006 
This document was prepared as an account of work sponsored by an agency of the United States Government. Neither the United States Government nor the University of California nor any of their employees, makes any warranty, express or implied, or assumes any legal liability or responsibility for the accuracy, completeness, or usefulness of any information, apparatus, product, or process disclosed, or represents that its use would not infringe privately owned rights. Reference herein to any specific commercial product, process, or service by trade name, trademark, manufacturer, or otherwise, does not necessarily constitute or imply its endorsement, recommendation, or favoring by the United States Government or the University of California. The views and opinions of authors expressed herein do not necessarily state or reflect those of the United States Government or the University of California, and shall not be used for advertising or product endorsement purposes.

This work was performed under the auspices of the U.S. Department of Energy by University of California, Lawrence Livermore National Laboratory under Contract W-7405-Eng-48. 


\title{
Experimental determination of drag coefficients in low-density polyurethane foam
}

\author{
Mark L. Adams* \\ University of California, Lawrence Livermore National Laboratory, P.O. Box 808, Livermore, CA 94551, USA
}

(Dated: April 15, 2006)

\begin{abstract}
We describe several experiments performed at the LLNL Site 300 firing range and on the LLNL $1 / 3$ scale gun to investigate the deceleration of small projectiles $(l \sim 3-5[\mathrm{~mm}])$ in low-density foam $\left(\rho \sim 0.08-0.32\left[\mathrm{~g} / \mathrm{cm}^{3}\right]\right)$. The experiments at the firing range researched a passive velocity diagnostic based on Faraday's law of induction, while experiments on the $1 / 3$ scale gun investigated the effects of varying projectile surface area, projectile shape, and foam density on the drag coefficient $c_{d}$. Analysis shows that the velocity diagnostic has an uncertainty on the order of 1 percent for projectiles with velocity $v \sim 0.8-1.2[\mathrm{~km} / \mathrm{s}]$. The $1 / 3$ scale gun experiments, dubbed the Krispy Kreme series, included nine shots considering the combinations of 3 projectile surface areas with 3 target densities. The experiments used Tantalum square surface area block projectiles (with an initial velocity $v_{0} \sim 1.2[\mathrm{~km} / \mathrm{s}]$, a common thickness $T=2.67[\mathrm{~mm}]$, and square side lengths of 3 , 4, and $5[\mathrm{~mm}]$ ) decelerating in polyurethane foams (with densities $\rho_{f}$ of $0.08,0.16$ and $0.32\left[\mathrm{~g} / \mathrm{cm}^{3}\right]$ ). Standard fluid models of the Krispy Kreme experiments predict Reynolds numbers $\operatorname{Re} \sim 10^{5}-10^{6}$, Mach numbers Ma $\sim 0.5-2.0$, and drag coefficients $c_{d} \sim 2-3$. However, the data indicate that $c_{d}=1.1-1.2\left(c_{d}=1.7\right)$ for all three block projectiles in the 0.08 and $0.16\left[\mathrm{~g} / \mathrm{cm}^{3}\right]$ targets $\left(0.32\left[\mathrm{~g} / \mathrm{cm}^{3}\right]\right.$ target $)$. First, we conclude that the drag force on projectiles in solid polyurethane foam is less than in fluids with equivalent dimensionless parameters. This result is also supported by an additional Krispy Kreme experiment that used a disk projectile (with diameter $d=4.51[\mathrm{~mm}]$ and thickness $T=2.67[\mathrm{~mm}])$ penetrating a target with density $\rho=0.16\left[\mathrm{~g} / \mathrm{cm}^{3}\right]$, i.e., the fluid-like $c_{d}=1.15$ while the measured $c_{d}=0.63$. Second, we conclude that the measured drag coefficient in the lower density foam targets is less than in the larger density foam target. This result is corroborated by firing range experiments with M855 NATO bullets (with diameter $5.56[\mathrm{~mm}]$, mass $4.1[\mathrm{~g}]$, and initial velocity $\left.v_{0} \sim 800[\mathrm{~m} / \mathrm{s}]\right)$ fired from an M16 rifle, i.e., $c_{d}=0.3\left(c_{d}=0.6\right)$ in the $0.16\left[\mathrm{~g} / \mathrm{cm}^{3}\right]\left(0.32\left[\mathrm{~g} / \mathrm{cm}^{3}\right]\right) \operatorname{target}$. We propose future $1 / 3$ scale gun experiments using a passive velocity diagnostic with increased spatial resolution as well as simple spherical projectiles with 0.5 and $1.0[\mathrm{~cm}]$ diameters.
\end{abstract}

\section{INTRODUCTION}

People have studied the deceleration of bodies in motion since the beginning of time. Around 25,000 BC, Neanderthals constructed elaborate cave drawings to depict their research on the deceleration of spears in flight during hunting expeditions. While this is obviously an exaggeration, our modern experimental and theoretical understanding of the forces on bodies moving through fluids is now more than a century old.

Around the turn of the $20^{\text {th }}$ century two major scientific advances in fluid dynamics occurred. First, the Wright Brothers performed novel wind tunnel tests to investigate lift and drag forces on various scaled airplane wing designs. ${ }^{1}$ Second, Ludwig Prandtl introduced the concept of a boundary layer and opened the door to theoretical drag calculations. [1] While both works have profoundly influenced modern approaches to the study of fluid mechanics, their application to projectiles moving through non-fluids is not straightforward.

\footnotetext{
*Electronic address: mladams@linl.gov

${ }^{1}$ See the Smithsonian National Air and Space Museum exhibit, "The Wright Brothers: The Invention of the Aerial Age."
}

In this paper we consider the deceleration of metal projectiles penetrating low-density polyurethane foams. From a theoretical perspective based on Prandtl's work, although a boundary layer must exist, performing theoretical drag calculations using a no-slip boundary condition at the projectile surface and assuming the boundary layer merges into an inviscid continuum is questionable at best. From an experimental perspective based on the Wright Brother's work, performing wind tunnel tests would require the design of scaled experiments that include solid material properties. Since these problems are worthy of their own study and appear to be one step removed from the current objective, we will simply conduct experiments that passively measure the change in velocity as a projectile penetrates a foam target.

Although the foam is a solid, research on the penetration of shaped charge jets in solid targets has shown that aspects of the projectile penetration will be fluidlike if the pressure is greater than the yield strength in the foam. [2] Assuming a projectile velocity of $v \sim$ $1.0[\mathrm{~km} / \mathrm{s}]$ and adopting the General Plastics foam density notation of Table I, i.e., 5\# (10\#) [20\#] refers to the foam with density $\rho_{f}=0.08(0.16)[0.32]\left[\mathrm{g} / \mathrm{cm}^{3}\right]$, the foam pressures $\left(p=\frac{1}{2} \rho_{f} v^{2}\right)$ are 40,80 , and 160 [MPa] for the 5\#, 10\#, and 20\# foam, respectively. Table I shows that the 5\#, 10\#, and $20 \#$ foam flexural strengths 
TABLE I: Properties of the General Plastics Manufacturing Co. LAST-A-FOAM FR-6700. Flexural modulus (FM) and flexural strength (FS) are defined by the ASTM D-790 standard. Sound speed $c_{s}$ is calculated using $c_{s}=\sqrt{F M / \rho_{f}}$, where $\rho_{f}$ is the density of the foam.

\begin{tabular}{ccccc}
\hline \hline & $\rho_{f}\left[\mathrm{~g} / \mathrm{cm}^{3}\right]$ & FM $[\mathrm{GPa}]$ & FS $[\mathrm{MPa}]$ & $c_{s}[\mathrm{~m} / \mathrm{s}]$ \\
\hline $5 \#$ & 0.08 & 0.026 & 1.0 & 570 \\
$10 \#$ & 0.16 & 0.088 & 3.0 & 745 \\
$20 \#$ & 0.32 & 0.290 & 8.2 & 952 \\
\hline \hline
\end{tabular}

are 1.0, 3.0, and $8.2[\mathrm{MPa}]$, respectively. Thus, the pressure is much greater than the yield strength and from shaped charge jet penetration research we expect the projectile penetration rate to be proportional to the stagnation pressure. In the next section the drag coefficient is introduced as the dimensionless proportionality constant.

The remainder of this paper proceeds as follows. Section II derives an equation for the drag coefficient, which is applied to the experimental data collected in this paper, and highlights important parameters. Section III presents Site 300 firing range experiments that tested both the fluid-like penetration hypothesis and the feasibility of a passive velocity diagnostic. Section IV presents $1 / 3$ scale gun experiments that studied the effects of varying projectile surface area, projectile shape, and foam density on the drag coefficient. Section V presents additional Site 300 firing range experiments used to quantify uncertainties in the passive velocity diagnostic and an analysis of corresponding effects on the drag coefficient. Section VI contains a brief discussion and proposes future research. Appendices A thorough $\mathrm{C}$ contain experimental data and analysis results.

\section{DRAG COEFFICIENT}

In this section we use Bernoulli's equation and Newton's second law of motion to express the drag coefficient in terms of both known and experimentally determined quantities.

Starting with Bernoulli's equation along an arbitrary streamline from point $A$ to point $B$, we write

$$
p_{A}+\frac{1}{2} \rho_{f} v_{A}^{2}=p_{B}+\frac{1}{2} \rho_{f} v_{B}^{2},
$$

where $p(v)$ is the fluid pressure (velocity), $\rho_{f}$ is the fluid density, and gravitational effects have been ignored. Taking $B$ at the stagnation point on the projectile surface and $A$ at a distant point along the same streamline (see Fig. 1), the no-slip condition requires $v_{B}=0$ and if $A$ is sufficiently far from $B$ we can assume $p_{B} \gg p_{A}$. Under

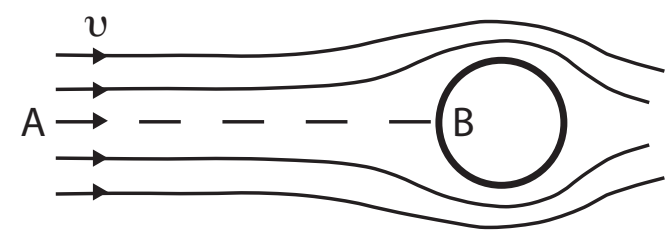

FIG. 1: This figure depicts streamlines around a spherical object moving with velocity $v$. The frame of reference is moving with the spherical object.

these assumptions, Bernoulli's equation reduces to

$$
p_{B}=\frac{1}{2} \rho_{f} v_{A}^{2} .
$$

In this paper the subscript $f$ refers to both fluid and foam.

Turning to Newton's second law of motion for a projectile with constant mass $m$ in the presence of a retarding drag force $F_{d}$, we write

$$
m \frac{d v}{d t}=-F_{d}=-c_{d} p A=-c_{d} \frac{1}{2} \rho_{f} v^{2} A,
$$

where $t$ is time, $v(A)$ is the projectile velocity (cross sectional area), $c_{d}$ is the dimensionless drag coefficient, and Eq. 2 has been used to eliminate pressure in the last equality. Now we let $m=\rho A T$, where $\rho(T)$ is the projectile density (characteristic thickness), and solve Eq. 3 for $\frac{d v}{d t}$ :

$$
\frac{d v}{d t}=-c_{d} \frac{1}{2 T} \frac{\rho_{f}}{\rho} v^{2}=-c_{d} \xi v^{2},
$$

where we defined $\xi=\frac{1}{2 T} \frac{\rho_{f}}{\rho}$.

Solving Eq. 4, with an initial velocity $v_{0}$ at time $t=0$, yields

$$
v=\frac{v_{0}}{1+v_{0} c_{d} \xi t} .
$$

Integrating once again, with an initial position $x=0$ at time $t=0$, yields

$$
x=\frac{1}{c_{d} \xi} \ln \left(1+v_{0} c_{d} \xi t\right) .
$$

Now, after solving Eq. 5 for $t(v)$ and substituting the result into Eq. 6, we can write

$$
v=v_{0} \exp \left\{-c_{d} \xi x\right\} .
$$

For a projectile with an initial velocity $v_{0}$ at position $x=$ 0 , Eq. 7 describes the exponential decrease in velocity $v$ after traveling a distance $x$. 
Finally, solving Eq. 7 for the drag coefficient yields

$$
c_{d}=\frac{-1}{\xi x} \ln \left(\frac{v}{v_{0}}\right) \text {. }
$$

All terms on the right side of Eq. 8 are either known in advance of an experiment or can be measured. In this paper, we measure $v(x)$ in experiments that vary projectile surface area, projectile shape, and foam density.

Before leaving this section let's consider one more equation. After returning to Eq. 5 and introducing a characteristic time $\tau$, i.e.,

$$
\tau=\frac{2 T}{v_{0} c_{d}} \frac{\rho}{\rho_{f}}
$$

we can write

$$
v=\frac{v_{0}}{1+\frac{t}{\tau}}
$$

From this equation it is clear that $v=\frac{1}{2} v_{0}$ when $t=\tau$.

\section{PRELIMINARY FIRING RANGE EXPEDITIONS}

Experiments at the LLNL Site 300 firing range were conducted on 16 December 2004 and 4 February 2005 to test the fluid-like penetration hypothesis and the feasibility of a passive velocity diagnostic.

The deceleration of metal projectiles penetrating lowdensity foams is dominated by the projectile stagnation pressure $p=\frac{1}{2} \rho_{f} v^{2}$, where $\rho_{f}$ is the foam density and $v$ is the projectile velocity. Furthermore, variations in pressure, e.g., due to differences in projectile shape and surface roughness, can be accounted for through the introduction of a dimensionless parameter called the drag coefficient $c_{d}$. This is what we are referring to as the fluid-like drag hypothesis. To test this hypothesis, we performed experiments that varied only the foam density and expected little variation in the drag coefficient (which is not necessarily unity). If there is no change in the drag coefficient, then the projectile deceleration rate scales with foam density $\rho_{f}$.

Our first set of experiments, which occurred on 16 December 2004, could best be described as a simple, quick, low cost, almost Neanderthal inspired, straightforward attempt to obtain reproducible drag coefficients. They involved taking a 20 [in] thick piece of 5\# polyurethane foam out to a field, firing an M16 assault rifle at it a few times, and measuring the exit velocity of the bullets with a chronograph (see Fig. 2). Table II displays the bullet exit velocities and corresponding drag coefficients calculated using Eq. 8. From these experiments we learned

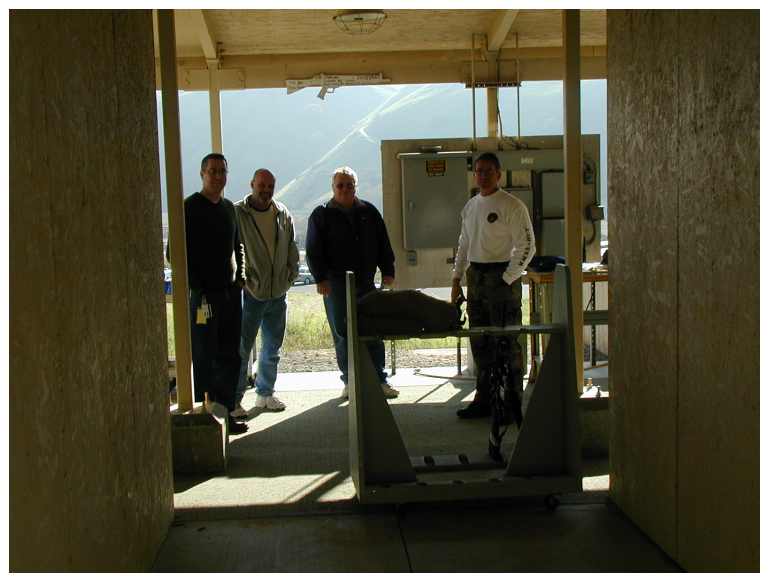

FIG. 2: Crew for the LLNL Site 300 firing range experiments on 16 December 2004 - the day we showed the foam who's boss.

that bullets tumble and the drag coefficient is very sensitive to the orientation of the bullet relative to the direction of motion. Analysis showed that an increase in the drag coefficient as the bullet tumbles could be explained by a decrease (increase) in projectile thickness (area). However, the main result of these experiments was that more data is needed.

To obtain more data per shot in future experiments we considered a passive version of a velocity diagnostic commonly implemented in shaped charge research. Foil switches are simple circuits that short when punctured. By staggering foil switches at know distances in a target, e.g., by sandwiching them between solid target plates, the velocity of shaped charge jets penetrating solid targets can be determined by $v=\Delta x / \Delta t$, where $\Delta x$ is the distance between two foil switches and $\Delta t$ is the difference in corresponding measured signal shorting times. Unfortunately, this approach is not feasible for the study

TABLE II: Exit velocities $v$ and drag coefficients $c_{d}$ from the LLNL Site 300 firing range experiments on 16 December 2004. These experiments used standard M855 NATO rounds (mass $m=4.1$ [g] $=62$ [grain] and diameter $d=5.56[\mathrm{~mm}])$ fired from an M16 rifle. The initial velocity $v_{0} \sim 2870$ [ft/s] was estimated from several null shots through a chronograph, the target was a 20 [in] thick piece of $\mathbf{5} \#$ foam, and $\rho T=m / A=16.89\left[\mathrm{~g} / \mathrm{cm}^{2}\right]$. In shot 3 the bullet started tumbling before exiting the foam target.

\begin{tabular}{cccc}
\hline \hline & shot 1 & shot 2 & shot 3 \\
\hline$v[\mathrm{ft} / \mathrm{s}]$ & 2813 & 2751 & 2486 \\
$c_{d}$ & 0.17 & 0.35 & 1.2 \\
\hline \hline
\end{tabular}




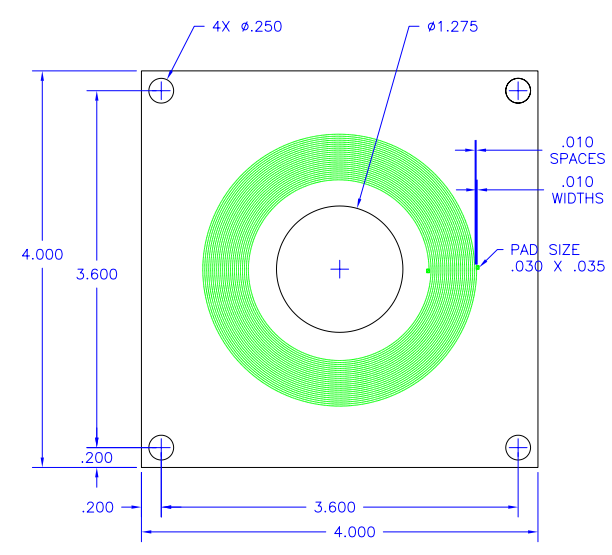

FIG. 3: Magnetic Donut circuit board. Magnetic Donuts were constructed by gluing a permanent donut shaped magnet to this circuit board.

of small projectiles penetrating low-density foam targets since the foil switches by themselves would introduce a significant rate of deceleration. Thus, we developed a detector, based on Faraday's law of induction, to passively determine projectile velocities in foam targets. [3]

Replacing the foil switches, our detector passively measures the change in magnetic field as a projectile passes the detector plane. Permanent donut shaped magnets generate the magnetic field and a simple circuit composed of 24 windings (see Fig. 3) measures the induced current. Construction of the detector involved mounting (gluing) the donut shaped magnet on the circuit board. Once forged the detector was instantly named the Magnetic Donut. Projectile velocities are determined by $v=\Delta x / \Delta t$, where $\Delta t$ is now the difference in Magnetic Donut signals times. The main advantage of this diagnostic is that it does not disturb the phenomenon begin measured.

Our second set of experiments at the LLNL Site 300 firing range, which occurred on 4 February 2005, tested the passive velocity diagnostic and colleted data on the deceleration of similar projectiles in foam targets with different densities. The projectiles were standard M855 NATO rounds fired from an M16. Figure 4 illustrates the experimental setup, which involved 4 Magnetic Donuts spaced 4 [in] apart and a chronograph placed in front of the apparatus. A total of 14 shots were performed, including 7 null shots $(1-4,7,13$, and 14), 3 shots with $10 \#$ foam targets $(5,6$, and 8$)$, and 4 shots with 20 \# foam tar- gets (9-12). ${ }^{2}$ Appendix A contains the Magnetic Donut signal time trace data and analysis of each shot.

Before discussing general experimental trends, we explore the data from shot 1 . Figure 8 contains 4 Magnetic Donut signal time traces that clearly indicate when the projectile is traversing the detector plane. The maximum absolute value of the signal is used to assign a specific time to each detector (in the $1 / 3$ scale gun experiments and later firing range experiments we used the time value between the maximum and minimum signal peaks that corresponded to the average of the extremium signals). Table VI lists projectile position and time histories. Table VII contains several velocities determined by $v_{i j}=\Delta x / \Delta t$, where $\Delta x=x_{j}-x_{i}\left(\Delta t=t_{j}-t_{i}\right)$ and the subscripts reference different Magnetic Donuts. For example, in shot 1 the projectile velocity obtained from Magnetic Donuts 1-2 is

$$
v_{12}=\frac{0.1016[\mathrm{~m}]-0.0[\mathrm{~m}]}{(5.76-4.48) \cdot 10^{-4}[\mathrm{~s}]}=793.75[\mathrm{~m} / \mathrm{s}] .
$$

The projectile velocities obtained from Magnetic Donuts 2-3 and 3-4 are $v_{23}=812.80[\mathrm{~m} / \mathrm{s}]$ and $v_{34}=$ $819.35[\mathrm{~m} / \mathrm{s}]$, respectively.

The velocities in shot 1 , which is a null experiment, are not identical. These differences must depend on variations in $\Delta x$ and $\Delta t$, i.e., their margins. Since other null shots, e.g., shot 3 , show a similar increase in projectile velocity across the diagnostic we conclude that the velocity variations are due to variations in $\Delta x$ from the assumed 4.0 [in]. Comparing the average velocity from the passive velocity diagnostic with the velocity obtained using a chronograph, the latter is consistently $20[\mathrm{~m} / \mathrm{s}]$ $(\sim 2.5 \%)$ slower. From repeatable shot performance and close agreement with an independent velocity diagnostic, we gained confidence in our passive velocity diagnostic.

Table V contains drag coefficients $c_{d}$ for the experiments containing foam targets. These values were calculated using Eq. 8 with velocity data from Table VII and initial information on the experimental setup. To illustrate this calculation, we consider the drag coefficient in shot 8 with projectile velocities obtained from Magnetic Donuts 1-2 $\left(v_{12}=846.67[\mathrm{~m} / \mathrm{s}]\right)$ and 2-3 $\left(v_{23}=839.67[\mathrm{~m} / \mathrm{s}]\right)$, i.e.,

$$
c_{d}=\frac{-2 T}{x} \frac{\rho}{\rho_{f}} \ln \left(\frac{v_{23}}{v_{12}}\right)=0.2117,
$$

where the projectile thickness is $T=0.019$ [m], the distance traveled in the foam is $x=0.1016$ [m], and the

\footnotetext{
${ }^{2}$ The numbers in parenthesis refer to shot numbers.
} 


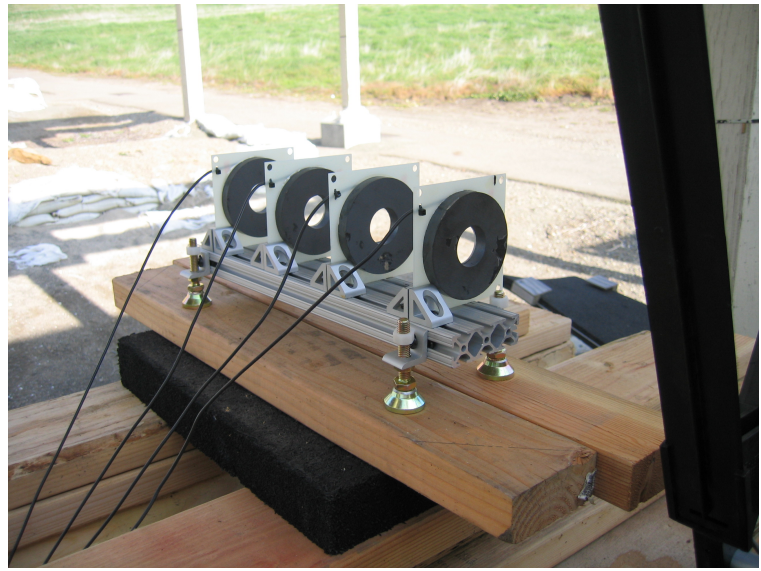

a) Magnetic Donut setup without form insert

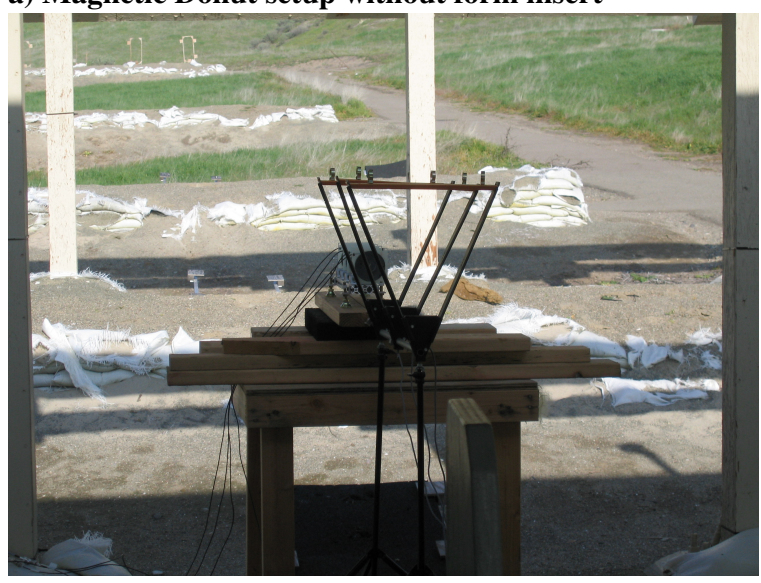

c) Orientation of Magnetic Donuts and chronograph

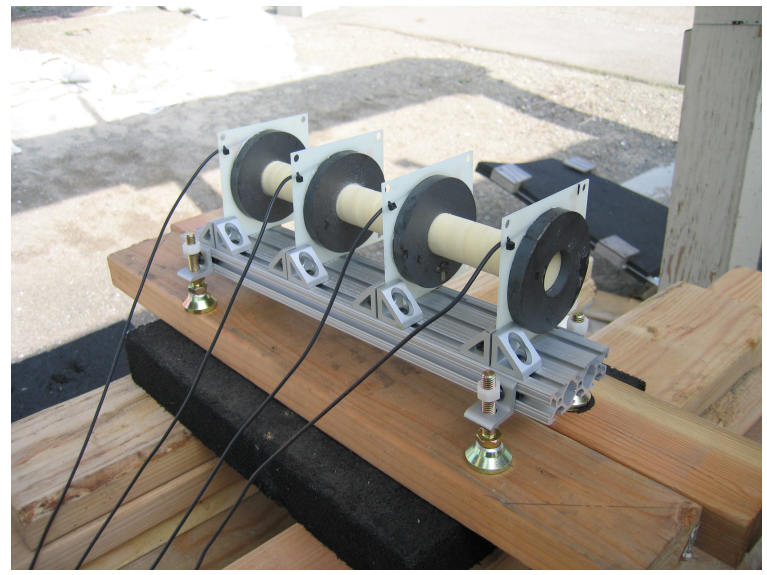

b) Magnetic Donut setup with foam insert

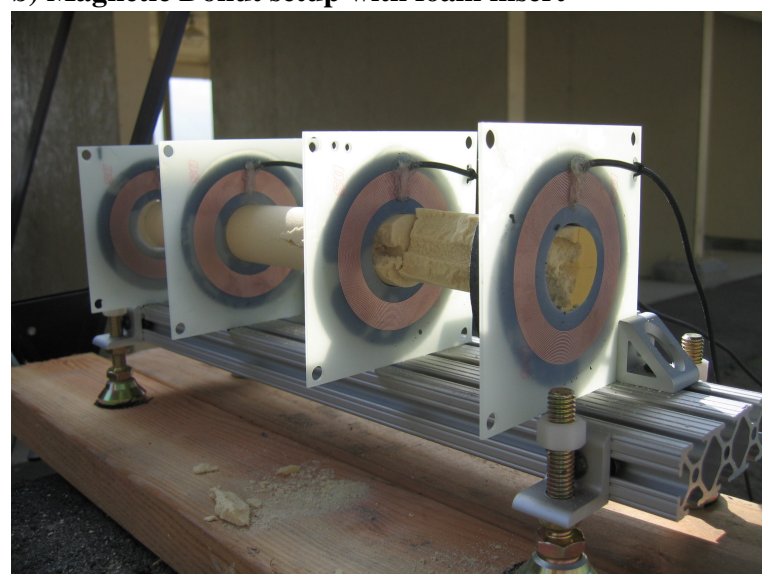

d) Rear view of the foam insert following a shot

FIG. 4: Photos from the LLNL Site 300 firing range experiments on 4 February 2005. The experimental setup involved 4 Magnetic Donuts spaced 4 [in] apart and a chronograph placed in front of the apparatus. In these experiments, the meager 1.275 [in] inner diameter of the Magnetic Donuts and 12 [in] target length introduced a high level of difficulty for the marksmen. Fortunately, due to the amazing skill of the marksmen, excellent data was collected.

foam (projectile) density is $\rho=0.16(11.0)\left[\mathrm{g} / \mathrm{cm}^{3}\right]$. Likewise, the drag coefficient in shot 8 with projectile velocities obtained from Magnetic Donuts 2-3 (1-2) and 3-4 (3-4) is $0.4183(0.3150)$. The drag coefficient obtained using $v_{12}$ and $v_{34}$ is an average of the drag coefficients obtained from the other two velocity combinations and is used to quote a single drag coefficient for each experiment.

For M855 NATO rounds fired from an M16 into 10\# (20\#) foam targets, the average drag coefficient $c_{d}$ is 0.315 (0.598). Roughly a factor of 2 differences in both the drag coefficients and the foam target densities exist. From these results we might conclude that the stagnation pressure scales as $\rho_{f}^{2}$, however future experiments will show this scaling is premature. Another possible expla- nation for this difference in drag coefficient is the scaling with Mach number. For example, in shot 8 (10\# foam target) $\mathrm{Ma} \sim 1.1$ while in shots 9-12 (20\# foam targets) $\mathrm{Ma} \sim 0.8$. However, in looking at the range of drag coefficient in shots 9-12, i.e., from 0.387 to 0.932 for the 10 values obtained in 20\# foam, a reproducible Mach number effect does not hold up. Due to the elongated bullet shape, variations in bullet orientation relative to the direction of propagation can easily account for this range of drag coefficients.

Although the range of drag coefficient in shots 9-12 is large, we find several comparable values where the bullet orientations are most likely similar. For example, the drag coefficients in shots 10 and 11 with projectile velocities obtained from Magnetic Donuts 1-2 and 2-3 are 

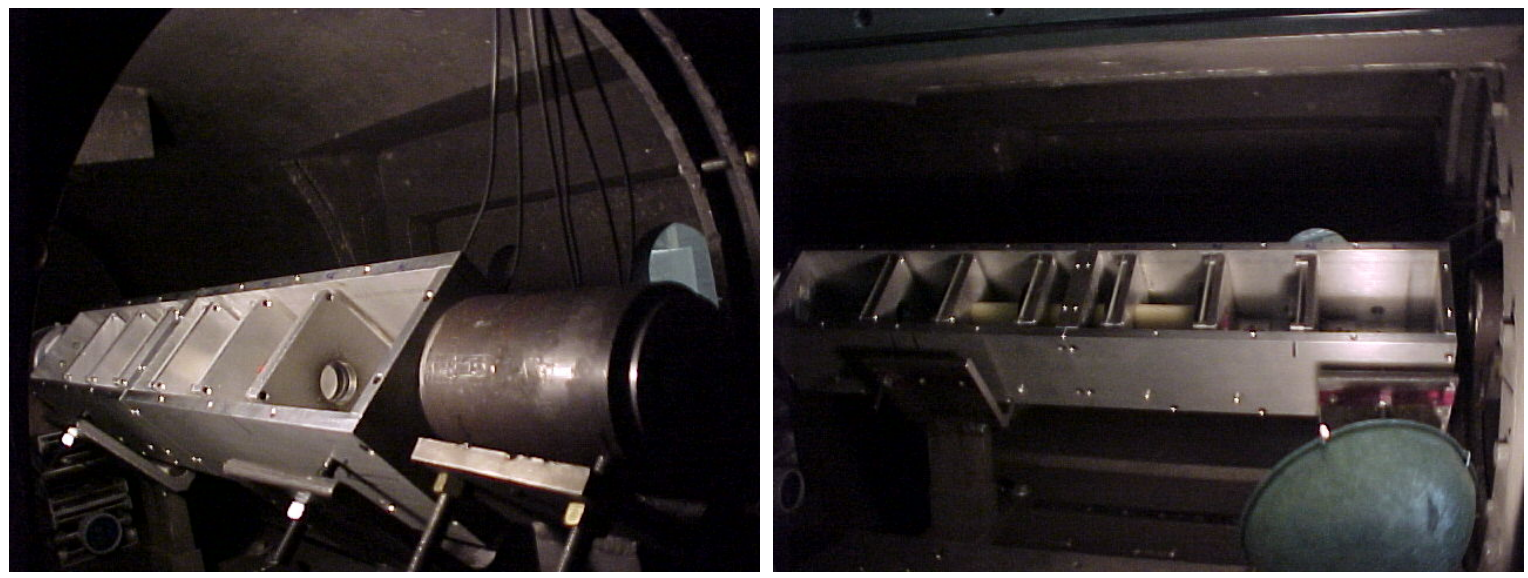

a) Alignment of Magnetic Donut apparatus and stripper b) Magnetic Donut setup with foam insert

FIG. 5: Photos from the LLNL 1/3 scale gun Krispy Kreme experiment on 1 April 2005. a) During an experiment the sabot and projectile exit the barrel (off the photo to the right) together, the sabot impacts the stripper (cylinder in the photo on the right) and ejects the projectile, which then traverses the Magnetic Donut apparatus (Aluminum rectangle in the photo on the left). b) This experiment fielded 6 Magnetic Donuts and the foam target was inserted between Magnetic Donut positions 3 through 6 (numbered from right to left in photo).

0.4926 and 0.4964 , respectively. Also, the drag coefficients in shots 9 and 11 with projectile velocities obtained from Magnetic Donuts 1-2 and 3-4 are 0.5463 and 0.5713 , respectively. Thus, we have some confidence in the fluid-like drag hypothesis and turn to a more controlled experimental environment.

\section{KRISPY KREME 1/3 SCALE GUN EXPERIMENTS}

Thirteen $1 / 3$ scale gun experiments, dubbed the Krispy Kreme series, were performed on 25 March 2005 through 8 April 2005. These experiments studied drag coefficient sensitivities to variations in projectile surface area and foam target density in a controlled environment.

The main projectiles in the Krispy Kreme experiments were Tantalum blocks with a common thickness $T=$ 2.67 [mm], square surface areas $A=l \times l$, and square side lengths $l$ of 3,4 , and 5 [mm]. The targets were General Plastics 5\#, 10\#, and 20\# polyurethane foams (see Table I). An additional Krispy Kreme experiment used a Tantalum disk projectile and 10\# foam target. The disk projectile had the same thickness and area as the $l=4[\mathrm{~mm}]$ block, i.e., disk diameter $d=4.51$ [mm]. Table III summarizes the Krispy Kreme series shots.

Figure 5 illustrates the general Krispy Kreme experimental setup. These experiments start by accelerating a plastic sabot, with the Tantalum projectile glued to the tip, down the $1 / 3$ stage gun pump tube (barrel). Upon exiting the barrel, the sabot impacts a device called the stripper and releases the projectile, i.e., the projectile is stripped from the sabot. The free projectile then traverses the Magnetic Donut apparatus.

Figure 6 illustrates the Magnetic Donut apparatus fielded in the Krispy Kreme experiments. To shield the Magnetic Donuts from both the plasma shock in front of the sabot and minimize the damage caused by wild projectiles, the apparatus fielded in previous firing range experiments (see Fig. 4) was significantly upgraded. This new apparatus was constructed using 3/8 [in] thick Aluminum plates and permits the fielding of 8 Magnetic Donuts. The Magnetic Donuts were numbered from the front, where the projectile enters, and foam targets were placed between Magnetic Donut positions 3 through 6 .

Drag coefficients are determined by the method outlined in the previous section. Since the plasma gas exiting the barrel introduces signal noise, the time value assigned to the Magnetic Donut signal corresponds to the average of the maximum and minimum signal peaks and is restricted to the domain defined by the maximum and minimum signal values. The signal in this region is smoother than near the signal peaks. Appendix B contains the Magnetic Donut signal time trace data, projectile fan plots, and detailed analysis of each shot.

Fan plots have been adopted from shaped charge research and are an interesting way to visualize projectile deceleration. For null experiments they appear as straight lines with a slope corresponding to the projectile velocity. For experiments involving projectile deceleration the slope decreases in time. 


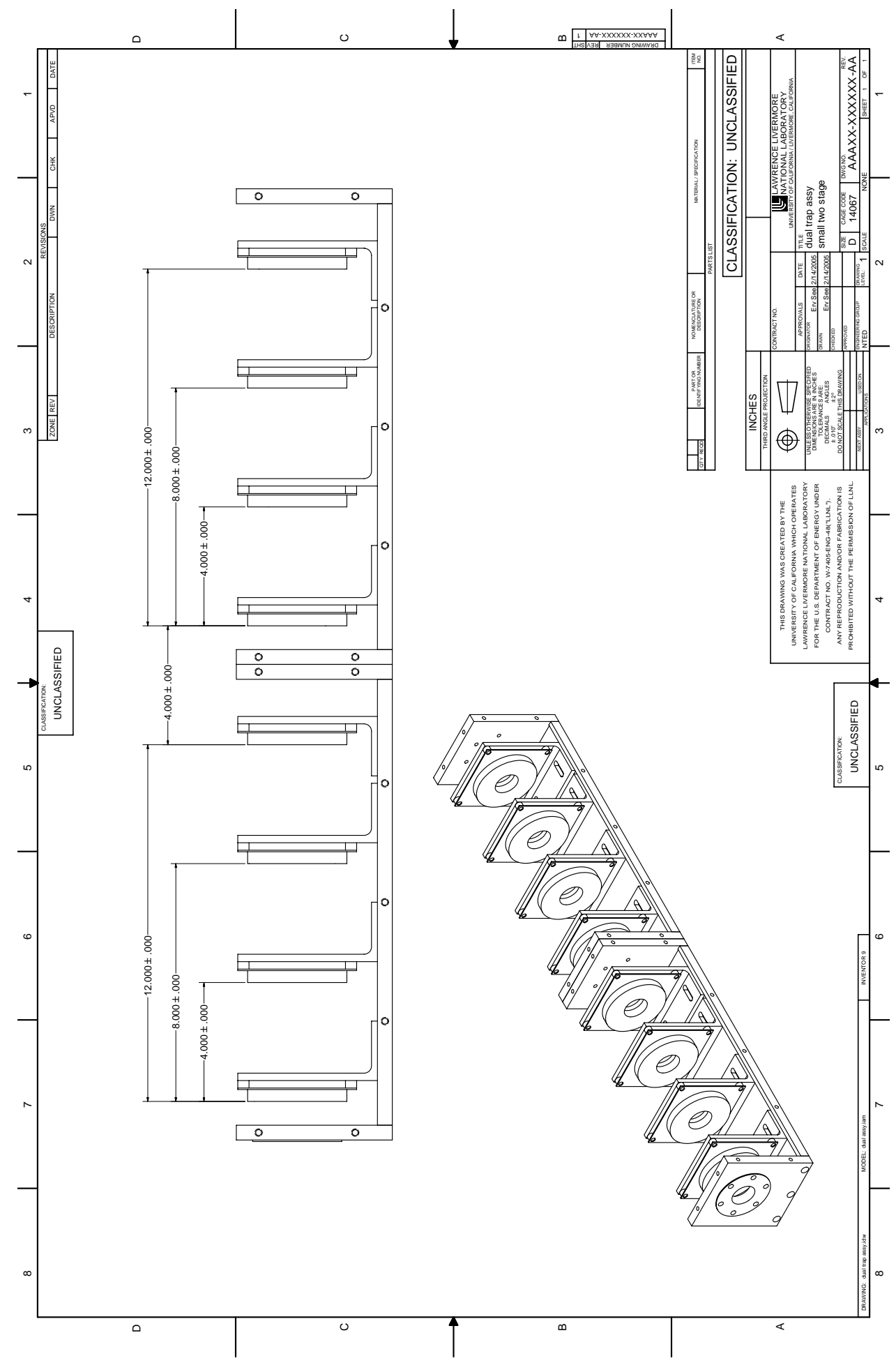

FIG. 6: Magnetic Donut apparatus in the Krispy Kreme experiments. This apparatus was constructed using 3/8 [in] thick Aluminum plates and designed to shield the Magnetic Donuts from both the plasma escaping the barrel and wild projectiles. 
TABLE III: Krispy Kreme shot summary.

\begin{tabular}{ccccc}
\hline \hline shot \# & date & projectile & target & comments \\
\hline 1392 & $3 / 25 / 05$ & $0.75[$ in] disk & none & \\
1393 & $3 / 29 / 05$ & $0.75[$ in] disk & none & \\
1394 & $3 / 30 / 05$ & $3[\mathrm{~mm}]$ block & $10 \#$ & no signal \\
1395 & $3 / 30 / 05$ & $9[\mathrm{~mm}]$ disk & none & \\
1396 & $3 / 31 / 05$ & $5[\mathrm{~mm}]$ block & $10 \#$ & \\
1397 & $4 / 1 / 05$ & $5[\mathrm{~mm}]$ block & $5 \#$ & $0.25[$ in] offset \\
1398 & $4 / 4 / 05$ & $4[\mathrm{~mm}]$ block & $5 \#$ & hit stripper \\
1399 & $4 / 5 / 05$ & $4[\mathrm{~mm}]$ block & $10 \#$ & \\
1400 & $4 / 6 / 05$ & $4.51[\mathrm{~mm}]$ disk & $10 \#$ & \\
1401 & $4 / 6 / 05$ & $3[\mathrm{~mm}]$ block & $5 \#$ & \\
1402 & $4 / 7 / 05$ & $3[\mathrm{~mm}]$ block & $20 \#$ & \\
1403 & $4 / 8 / 05$ & $4[\mathrm{~mm}]$ block & $20 \#$ & \\
1404 & $4 / 8 / 05$ & $5[\mathrm{~mm}]$ block & $20 \#$ & \\
\hline \hline
\end{tabular}

TABLE IV: Krispy Kreme drag coefficient summary.

\begin{tabular}{|c|ccc|}
\hline \hline$l[\mathrm{~mm}]$ & $5 \#$ & $10 \#$ & $20 \#$ \\
\hline 3 & 1.10 & 1394 & 1.98 \\
4 & 1398 & 1.01 & 1.53 \\
5 & 1397 & 1.40 & 1.68 \\
\hline
\end{tabular}

Table IV summarize the drag coefficients obtained in the Krispy Kreme experiments that varied block projectile surface areas and foam target densities. While the Magnetic Donut apparatus and foam target arrangement allows for two initial and final velocity measurements, due to target movement following projectile impact we focus on the drag coefficient calculations using the projectile velocities obtained from Magnetic Donuts 3-4, 45 , and 5-6. The drag coefficient obtained using $v_{34}$ and $v_{56}$ is an average of the drag coefficients obtained from the other two velocity combinations and is quoted in Table IV when available.

The Krispy Kreme experimental results show that drag coefficients in low-density polyurethane foams are less than fluids with equivalent dimensionless parameters. With velocity $v \sim 10^{3}[\mathrm{~m} / \mathrm{s}]$, length $l \sim 10^{-3}[\mathrm{~m}]$, and kinematic viscosity $\nu \sim 10^{-6}\left[\mathrm{~m}^{2} / \mathrm{s}\right]$, these experiments have Reynolds number is $\operatorname{Re} \sim v l / \nu \sim 10^{6}$. Referring to a fluid dynamics handbook [4], the drag coefficient for block projectiles with aspect ratios $T / l=0.89,0.67$, and 0.53 (i.e., the the 3,4 , and 5 [mm] projectiles) are 2.3, 2.9 , and 2.5 , respectively. For $\operatorname{Re}>10^{4}$ the drag coefficient variation with aspect ratio peaks near $T / l=0.65$. These values are all significantly larger than the values in Table IV.

One possible explanation for this difference in drag coefficients is a dependence on Mach number. However, drag coefficients typically increases for velocities around the sound speed, e.g., a cube with $c_{d} \sim 2.1$ for $\mathrm{Ma}<0.8$, quickly rises to $c_{d} \sim 2.6$ for $\mathrm{Ma}=0.85$, and continues to increase as the Mach number approaches unity. Another possible explanation of this difference in drag coefficients is a dependence on projectile orientation. However, variations in cube orientation amounts to a range of drag coefficients between 1.8 and 2.4.

This decrease in drag coefficient compared to equivalent fluid values is further supported by another Krispy Kreme experiment involving the disk projectile in 10\# foam. The fluid drag coefficient is $c_{d}=1.15$ while the measured value is $c_{d}=0.68$ (see data from shot 1400).

Table IV also depicts an increase in the average drag coefficient with foam target density, i.e., $c_{d}=1.1,1.2$, and 1.7 in the 5\#, 10\#, and 20\# foam, respectively. While this suggests pressure scalings of $\rho_{f}^{4 / 3}$ or $\rho_{f}^{3 / 2}$, instead of $\rho_{f}$, more data is needed to support such a conclusion.

\section{VELOCITY DIAGNOSTIC UNCERTAINTY EXPERIMENTS AND DRAG COEFFICIENT ERROR ESTIMATES}

Experiments at the LLNL Site 300 firing range were conducted on 13 April 2005 to quantify uncertainties in the passive velocity diagnostic used in the Krispy Kreme experiments. With velocity diagnostic uncertainties, drag coefficient error estimates can be performed.

Twelve identical null experiments were performed using M855 NATO rounds fired from an M16 and the Krispy Kreme Magnetic Donut apparatus. Although 8 Magnetic Donuts were fielded in these experiments, only the first four signals were recorded. Also, Magnetic Donut signal traces in shots 1 and 12 were not recorded. Appendix C contains the Magnetic Donut signal time trace data and detailed analysis of each shot.

In reality, the bullet velocity in these null experiments is constant across the Magnetic Donut apparatus, i.e., air produces little deceleration on the bullets as they traverse the apparatus. Thus, given $\Delta t$ from the Magnetic Donut signals we can adjust the individual Magnetic Donut positions $x_{i}$ so that $\Delta x$ yields a constant velocity according to $v=\Delta x / \Delta t$. This produces a set of corrections $\delta_{i}$ for each position $x_{i}$. Now if the average correction $\bar{\delta}$ is subtracted from each new position, i.e, $x_{i}+\delta_{i}-\bar{\delta}$, then a best estimate of the constant velocity is obtained. This velocity can be used to quantify the variation in velocities determined by the uncorrected Magnetic Donut apparatus.

Figure 7 plots the normalized distribution of the difference between the best constant velocity for each shot and the corresponding uncorrected velocities in Table XII. The accuracy of the constant velocities is reflected in the 


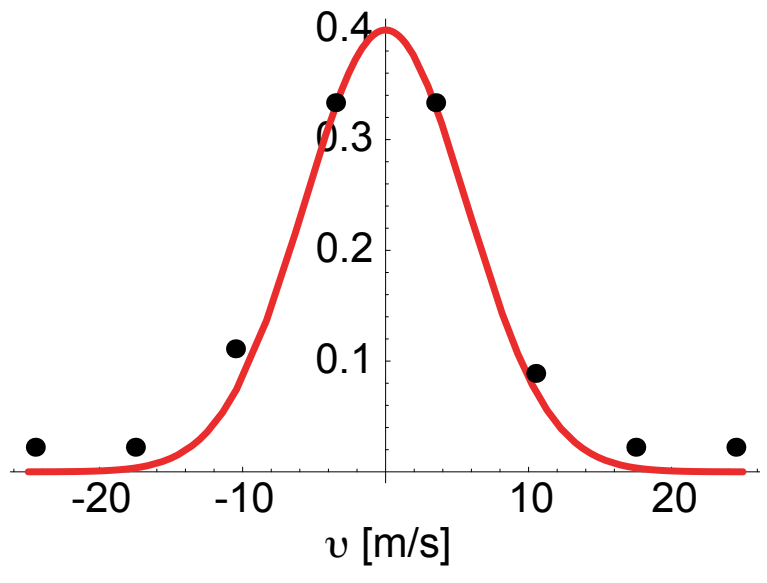

FIG. 7: Gaussian fit (solid line) to the normalized distribution of velocity differences in the LLNL Site 300 firing range experiments conducted on 13 April 2005 (circles). The Gaussian curve has a standard deviation of 11.381 [m/s].

distribution peak near $v=0[\mathrm{~m} / \mathrm{s}]$. The uncertainty of the velocity diagnostic is given by the spread in the distribution about the peak. The standard deviation of the 45 velocity data points in Table XII is $11.381[\mathrm{~m} / \mathrm{s}]$ and Fig 7 plots the corresponding Gaussian distribution. Since the average bullet velocity is $v \sim 800[\mathrm{~m} / \mathrm{s}]$ the uncertainty in our velocity diagnostic is $\sim 1 \%$.

Given an uncertainty in velocity $\epsilon$, we can estimate the effect on the drag coefficient. Let $v \rightarrow v \pm \epsilon$ in Eq. 8:

$$
c_{d}=\frac{-1}{\xi x} \ln \left(\frac{v \pm \epsilon_{1}}{v_{0} \pm \epsilon_{2}}\right) .
$$

Assuming $\epsilon_{1}=\epsilon_{2}=\epsilon$, considering the positive variation, and expanding Eq. 13, we find:

$$
c_{d}=\frac{-1}{\xi x}\left[\ln \left(\frac{v}{v_{0}}\right)+\left(\frac{1}{v}-\frac{1}{v_{0}}\right) \epsilon\right] .
$$

Using the dimensionless values $\hat{v}_{0}=1, \hat{v}=\frac{1}{2}$, and $\hat{\epsilon}=$ 0.01 , we find the first (second) term in square brackets yields $-0.69(0.01)$. Thus, an uncertainty in the velocity diagnostic of order $1 \%$ produces a similar uncertainty in the drag coefficient.

For completeness, we consider the effect of margins in the foam thickness penetration length $x$. Let $x \rightarrow x \pm \epsilon$, where now $\epsilon$ is a variation in position, in Eq. 8:

$$
c_{d}=\frac{-1}{\xi(x \pm \epsilon)} \ln \left(\frac{v}{v_{0}}\right) .
$$

Considering the positive variation and expanding Eq. 15, we find:

$$
c_{d}=\frac{-1}{\xi x} \ln \left(\frac{v}{v_{0}}\right)\left[1+\frac{\epsilon}{x}\right] .
$$

Thus, a $1 \%$ variation in $x$, i.e., $\epsilon=0.01 x$, has a $1 \%$ effect on the drag coefficient.

\section{DISCUSSION}

More data is needed. This seems to be the common result of every experiment. In the Krispy Kreme experiments, although 5 relevant velocity points were collected and 4 drag coefficient calculations are possible, target movement introduced a significant uncertainty in the initial and final foam thickness lengths traveled by the projectile and only the center 3 velocities and 2 corresponding drag coefficients are valid. With an additional $1 / 3$ of the 9 main Krispy Kreme shots resulting in no data, it is difficult to draw sweeping conclusions. Thus, we humbly present the observed trends.

Data from the Krispy Kreme experiments show that block Tantalum projectiles with initial velocities $v_{0} \sim$ $1.2[\mathrm{~km} / \mathrm{s}]$ have drag coefficients $c_{d} \sim 1.1-1.2$ in polyurethane foams with densities $\rho_{f}=0.08$ and $0.16\left[\mathrm{~g} / \mathrm{cm}^{3}\right]$. For the same block projectiles the drag coefficient rises to approximately $c_{d} \sim 1.7$ in foams with density $\rho_{f}=0.32\left[\mathrm{~g} / \mathrm{cm}^{3}\right]$. These drag coefficients are all less than fluid models with similar dimensionless parameters predict, i.e., $c_{d} \sim 2.0-3.0$.

Several possible explanations that cannot explain this difference in measured and expected drag coefficients have been explored. First, velocity diagnostic uncertainties account for only a $3 \%(1 \%) 3(1)-\sigma$ uncertainty in the drag coefficient. Second, block projectile orientation sensitivities can account for only a $10 \%$ uncertainty in the drag coefficient. Finally, no obvious Mach number sensitivity was observed in the data with velocities straddling the sound speed, i.e., there is no obvious increase in drag coefficient when the Mach number was near unity. Thus, we argue that something interesting is happening in the boundary layer.

Let's consider a modification to Prandtl's boundary layer. Imagine a sphere projectile traveling through a foam target. Near the surface of the sphere, the pressure in the foam is greater than its yield strength so the foam will behave as a fluid and the no-slip boundary condition is appropriate at the projectile surface. At the other 
end of the boundary layer, there is not a transition to an inviscid continuum but rather a transition to a solid. The hole that remains in the foam following penetration supports this transition. Now there is another transition region, between the undisturbed solid and the projectile surface, which occurs where the pressure in the foam is proportional to its yield strength. On the projectile side of this layer we expect fluid flow, while on the solid side we expect elastic-plastic flow. Thus, since the transition layer moves toward the projectile as the target density increases, a plausible conjecture is that the density effect on the drag coefficient is due to an increase in wave drag as that the inner transition layer moves toward the projectile. Since the target hole diameter is inversely proportional to the target material strength, which increases with target density, an additional increase in wave drag occurs as the solid boundary moves toward the projectile.

Continuing with the more data is needed theme, we propose future experiments that significantly increase the number of drag coefficients obtained in a single shot and removes some of the ambiguity of the current experiments. For $N$ detectors in an experiment, we obtain $N-1$ velocity points and $N-2$ drag coefficients. In current experiments $N=4$; in future experiments $N=6$ would double the number of drag coefficients obtained while $N=12$ would results in a 5 -fold increase. To remove the drag coefficient sensitivity to projectile orientation in future experiments, we propose to use spherical Tantalum projectiles with diameters $d=0.5$ and $1.0[\mathrm{~cm}]$. We also propose an additional target density to assist in understanding the density scaling, i.e., we propose to use foam targets with densities $\rho_{f}$ of $0.08,0.16$, 0.32 , and $0.64\left[\mathrm{~g} / \mathrm{cm}^{3}\right]$.

\section{Acknowledgments}

Several people contributed to the success of these experiments. Monte Brandrup was instrumental in arranging the LLNL Site 300 firing range experiments, Dan Greenwood was responsible for rapid development of the Magnetic Donuts, and Neal Hinsey was in charge of coordinating the LLNL 1/3 scale gun experiments. This work also benefited from the input and support of Steve Bosson, Robert Claire, Nick Collier, Jeff Getz, Maria Hinsey, Omar Hurricane, Kevin Morris, Anthony Regalado, Charlie Verdon, Alan Wan, and Sam Weaver.

This work was performed under the auspices of the U.S. Department of Energy by the University of California, Lawrence Livermore National Laboratory through contract W-7405-ENG-48.
[1] Jr. John D. Anderson. Ludwig prandtl's boundary layer. Physics Today, 58(12):42, 2005.

[2] G. Birkhoff, D.P. MacDougall, E.M. Pugh, and G. Taylor. Explosives with lined cavities. Journal of Applied Physics, 19:563, 1948.

[3] R.S. Hawke, A.R. Susoeff, and D.W. Greenwood. Design considerations for a passive magnetic induction signal generator for sensing hypervelocity projectile passage. IEEE Transactions on Magnetics, 31(1):725, 1995.

[4] R.D. Blevins. Applied Fluid Dynamics Handbook. Van Nostrand Reinhold Company, New York, 1984. 
APPENDIX A: FIRING RANGE EXPERIMENTS ON 2 FEBRUARY 2005

FIG. 8: Range Day 2, Shot 1, Null Test
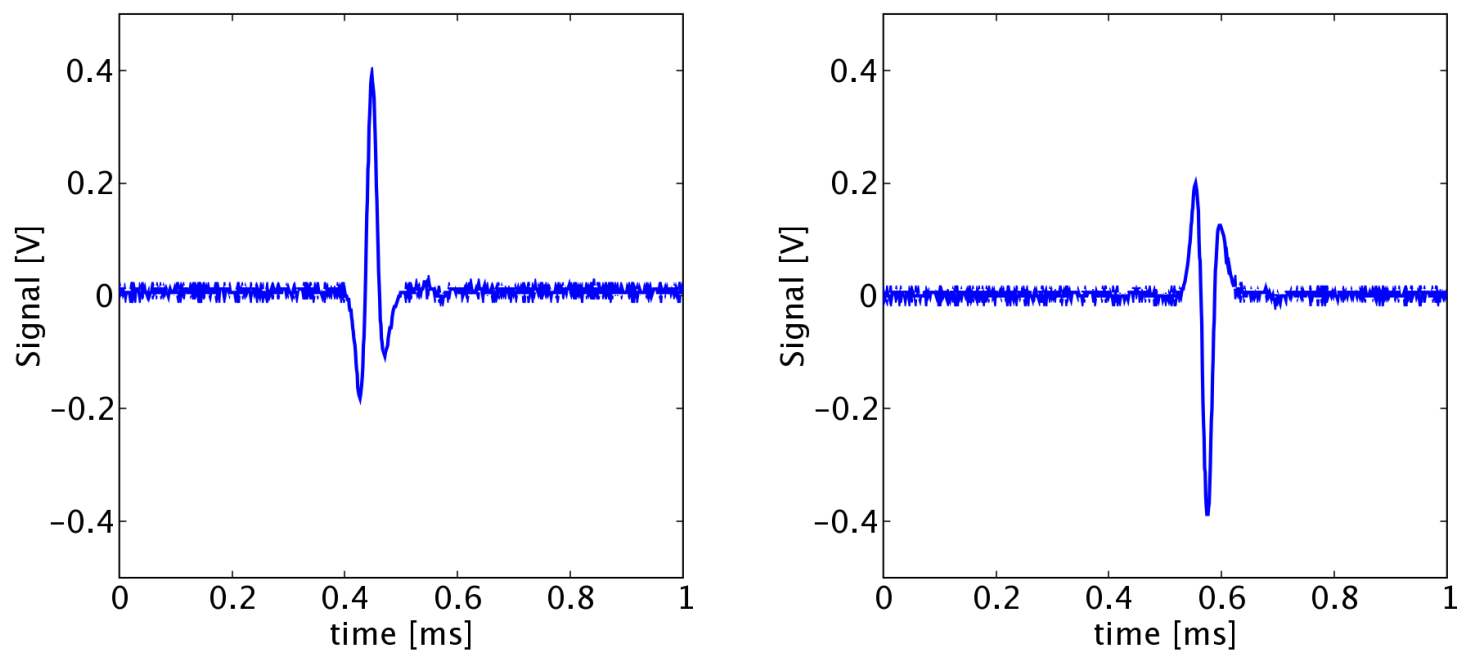

a) Signal from Magnetic Donut 1

b) Signal from Magnetic Donut 2
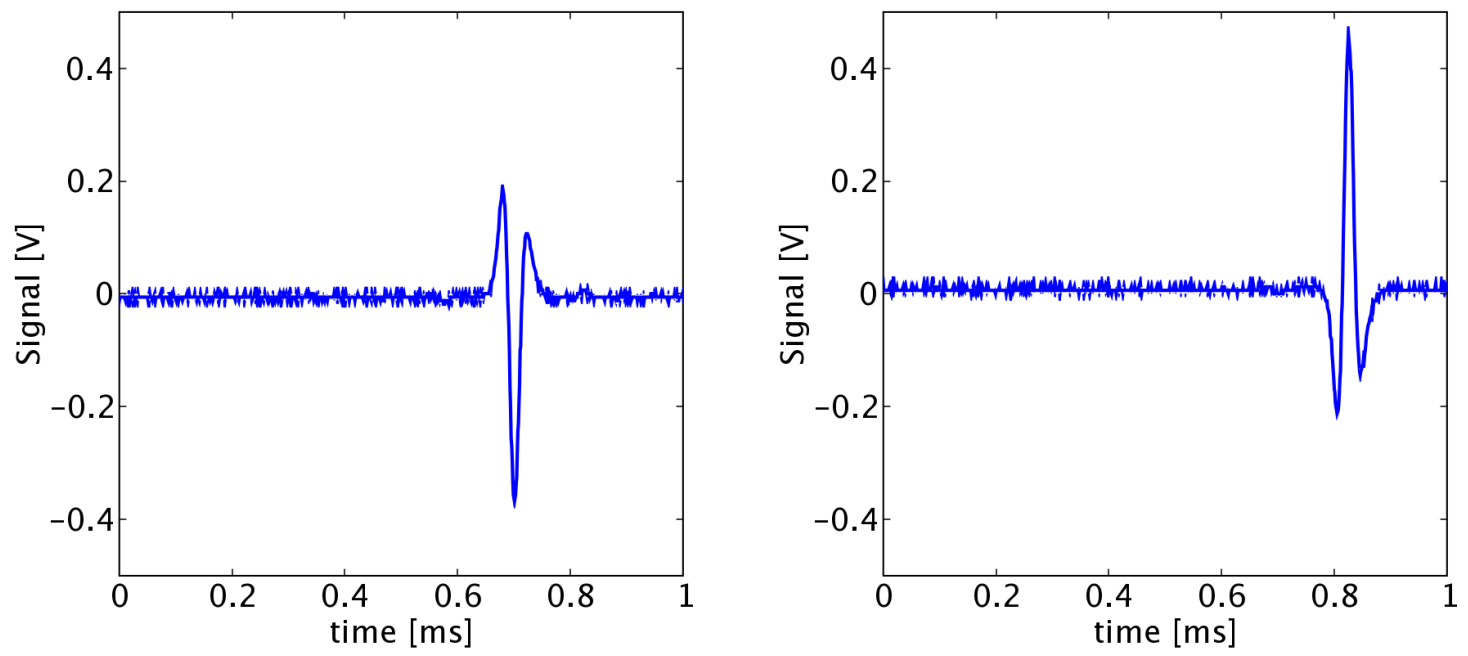

c) Signal from Magnetic Donut 3

d) Signal from Magnetic Donut 4 
FIG. 9: Range Day 2, Shot 2, Null Test

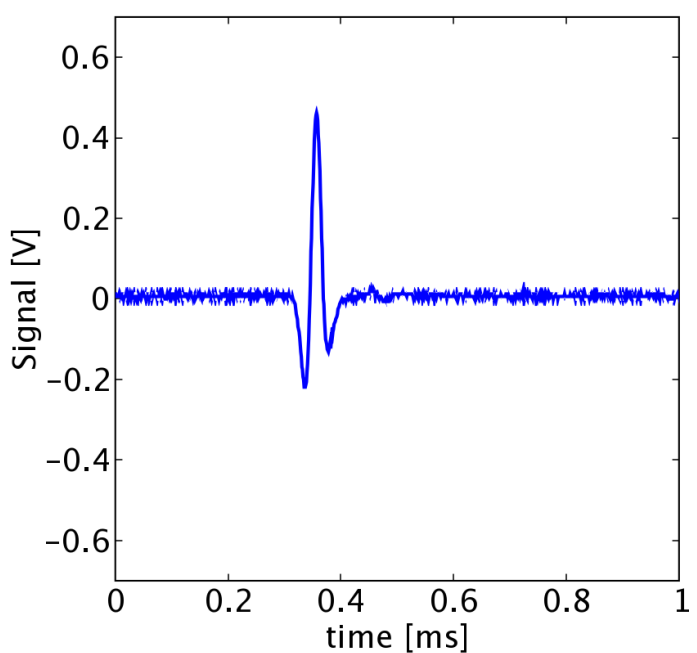

a) Signal from Magnetic Donut 1

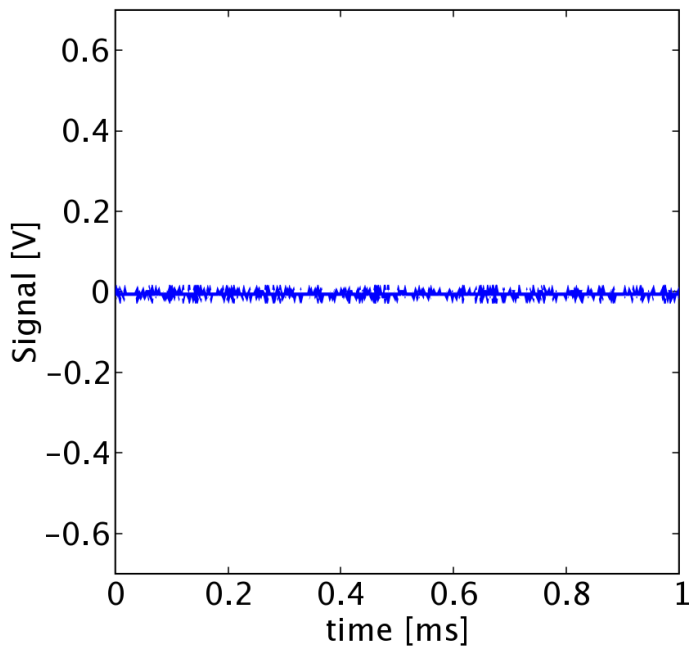

c) Signal from Magnetic Donut 3

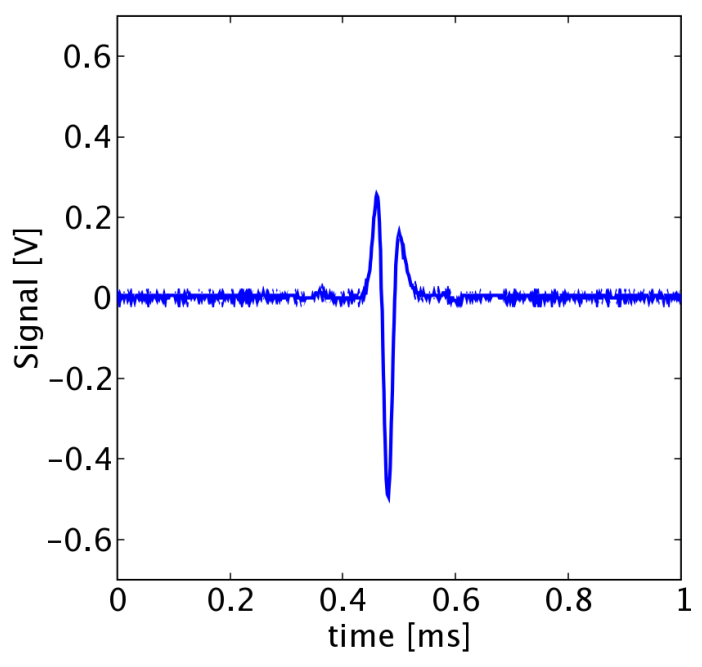

b) Signal from Magnetic Donut 2

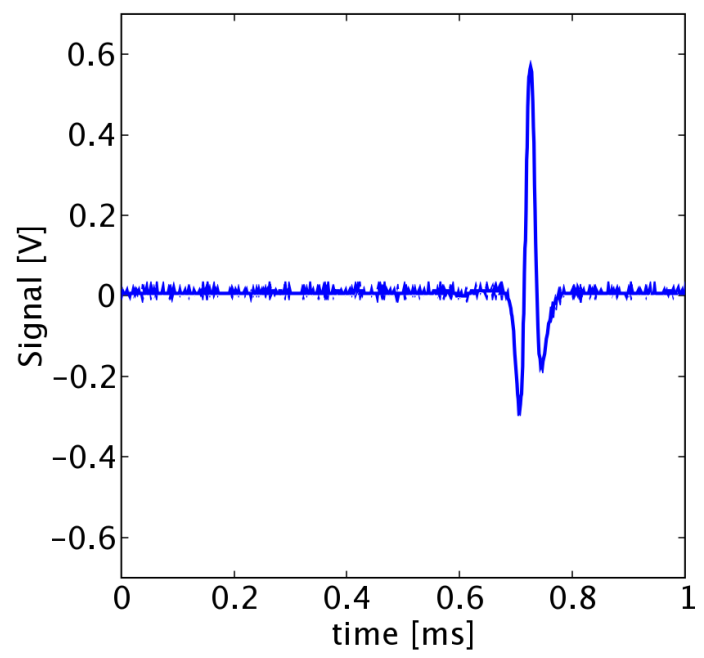

d) Signal from Magnetic Donut 4 
FIG. 10: Range Day 2, Shot 3, Null Test

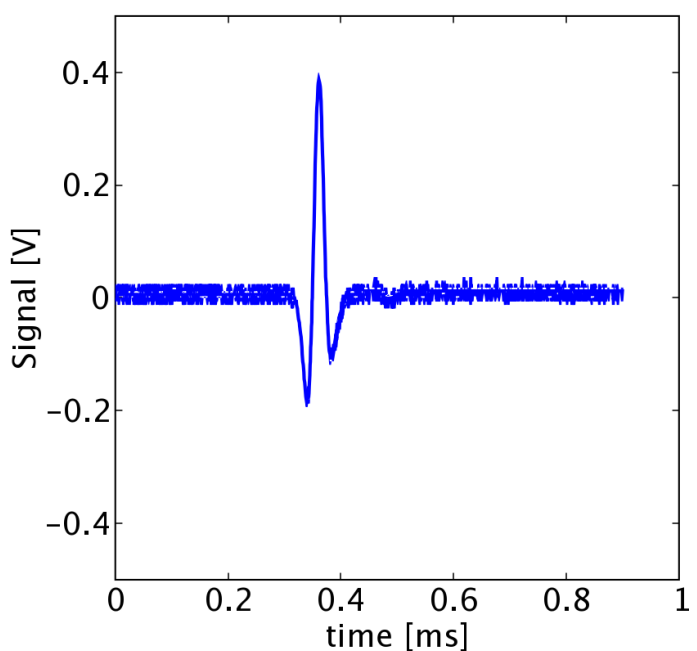

a) Signal from Magnetic Donut 1

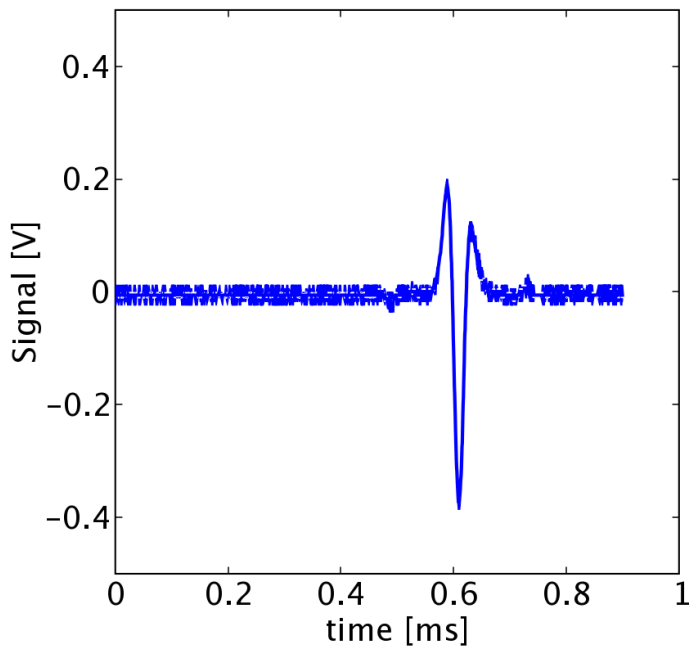

c) Signal from Magnetic Donut 3

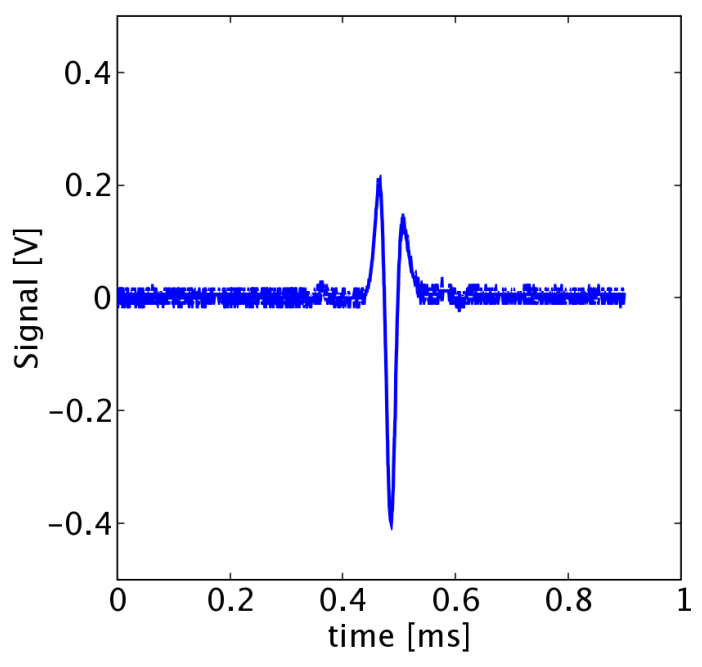

b) Signal from Magnetic Donut 2

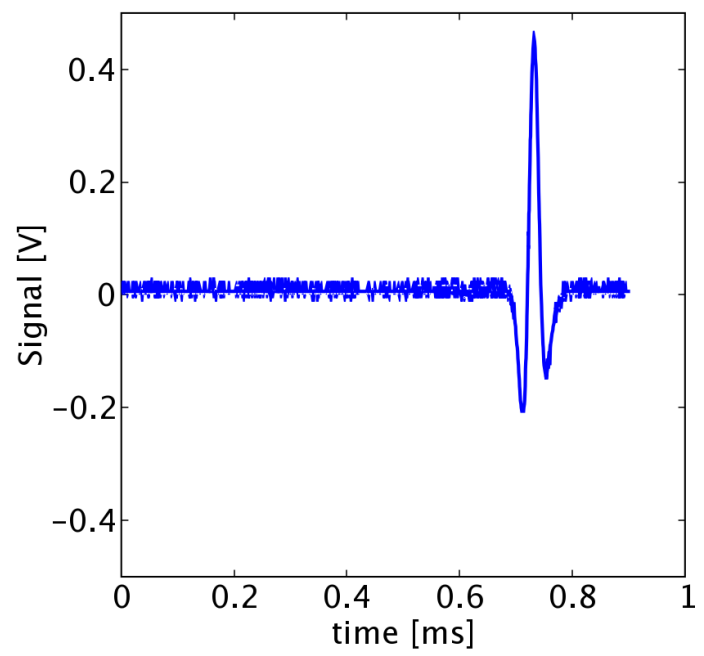

d) Signal from Magnetic Donut 4 
FIG. 11: Range Day 2, Shot 4, Null Test

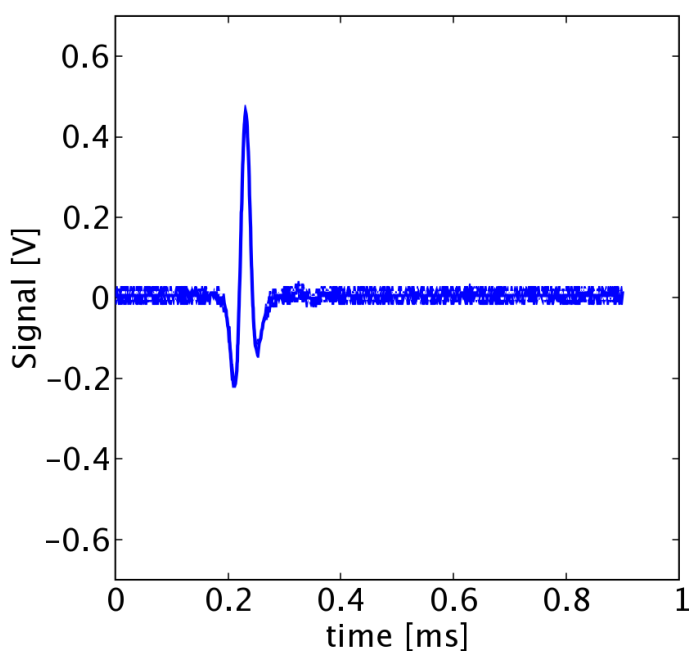

a) Signal from Magnetic Donut 1

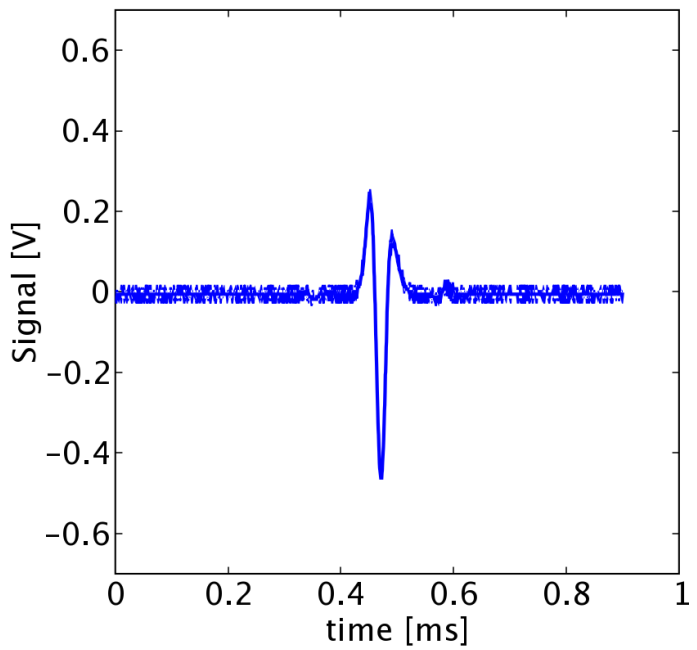

c) Signal from Magnetic Donut 3

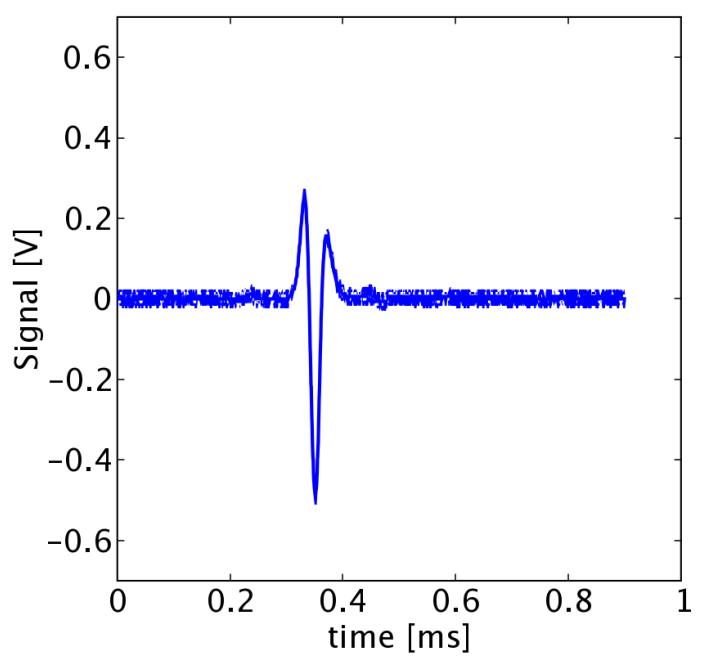

b) Signal from Magnetic Donut 2

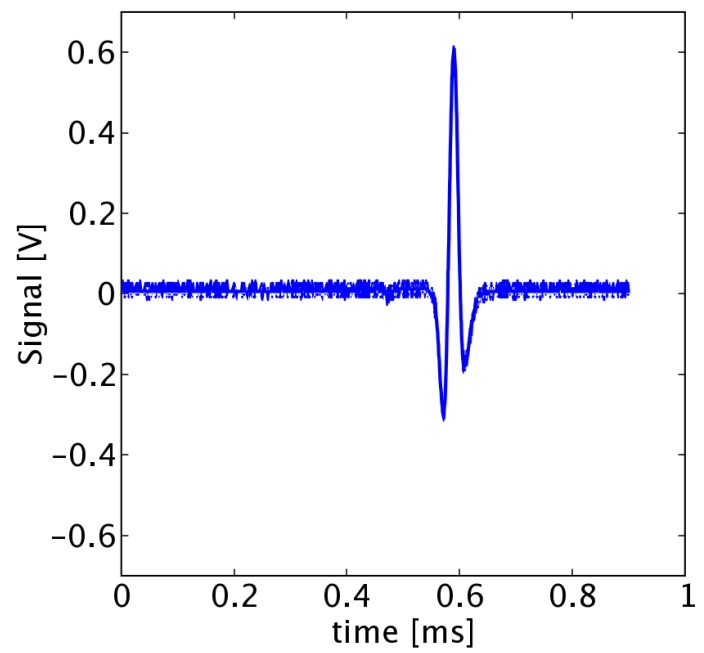

d) Signal from Magnetic Donut 4 
FIG. 12: Range Day 2, Shot 5, 10\# Foam

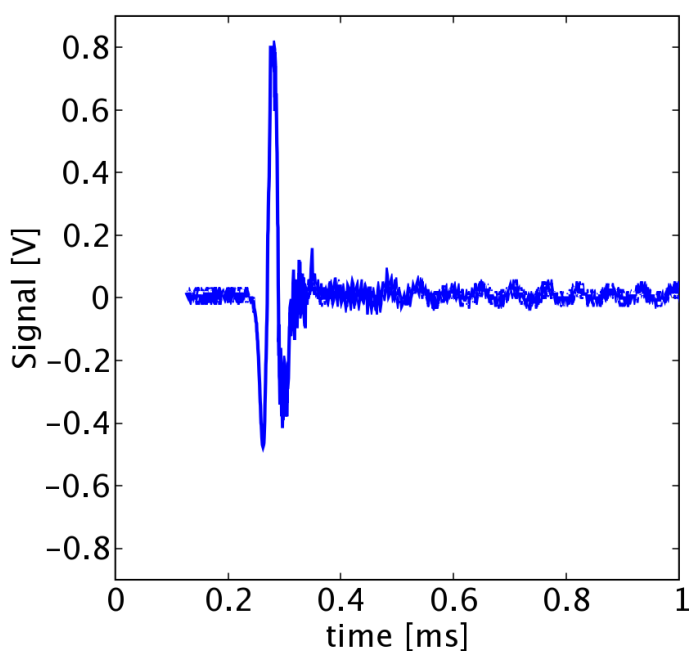

a) Signal from Magnetic Donut 1

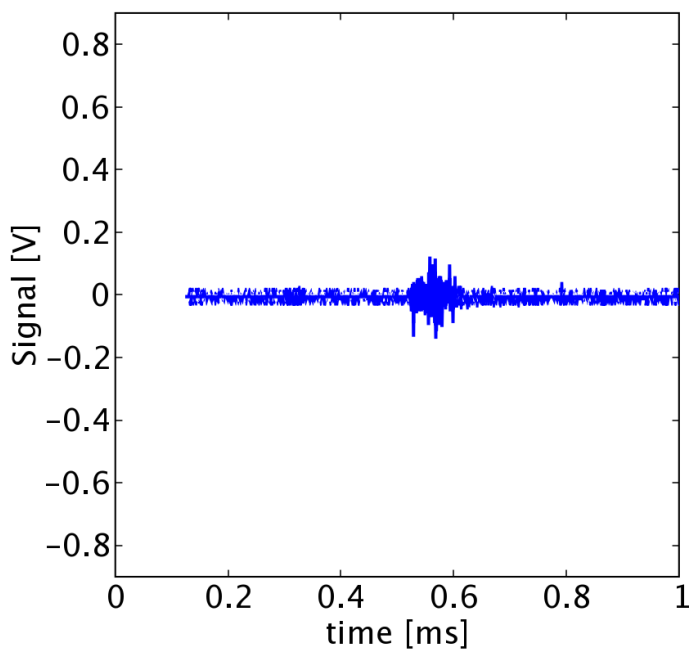

c) Signal from Magnetic Donut 3

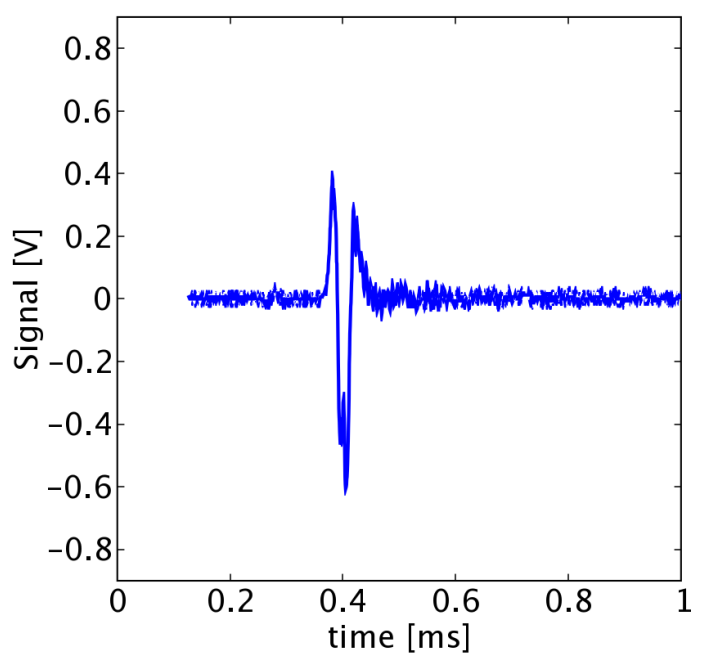

b) Signal from Magnetic Donut 2

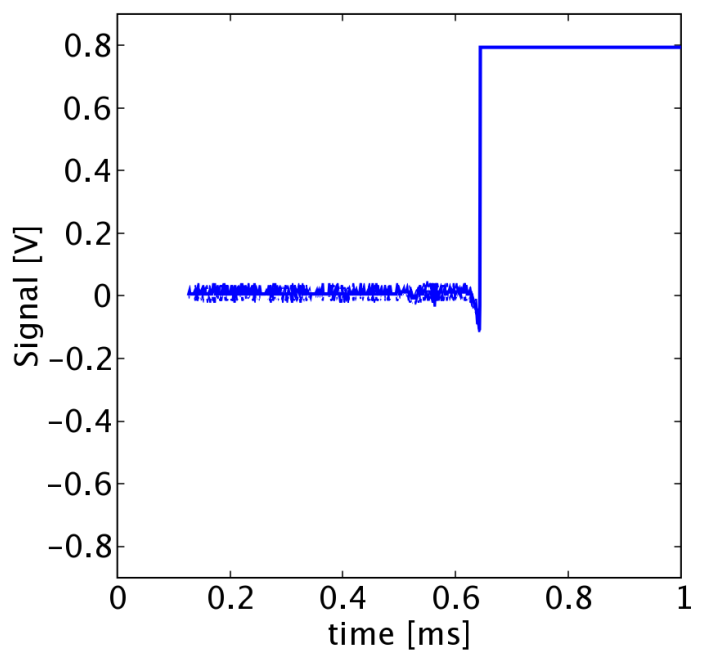

d) Signal from Magnetic Donut 4 
FIG. 13: Range Day 2, Shot 6, 10\# Foam

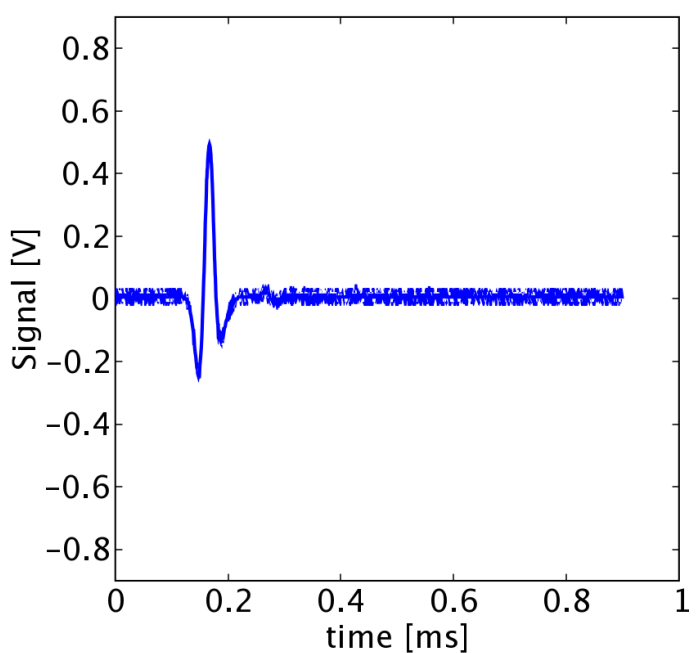

a) Signal from Magnetic Donut 1

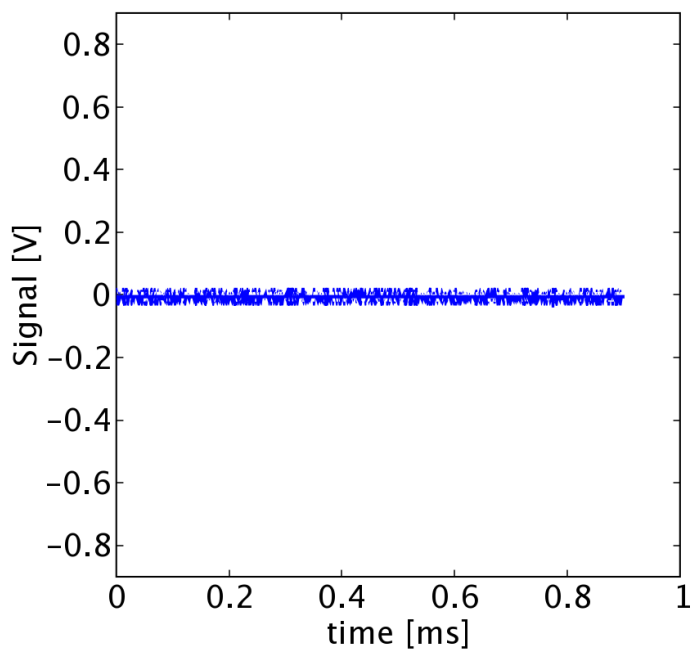

c) Signal from Magnetic Donut 3

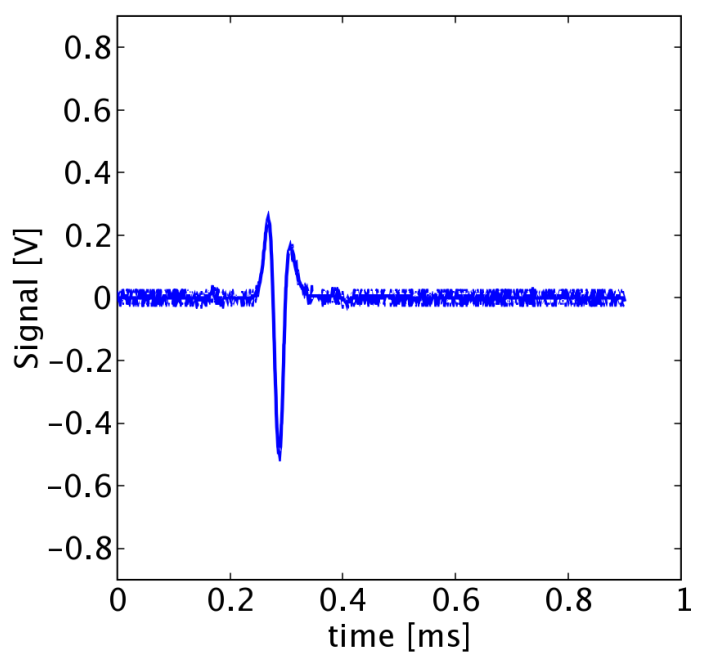

b) Signal from Magnetic Donut 2

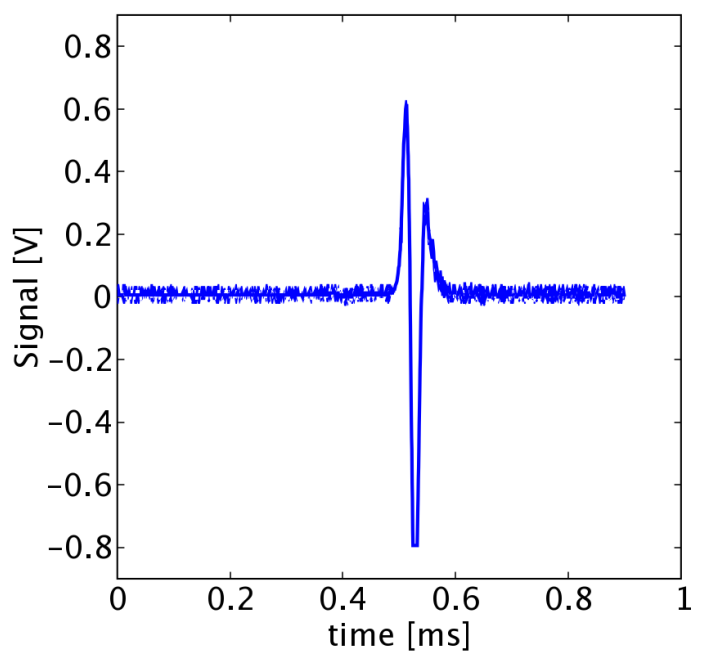

d) Signal from Magnetic Donut 4 
FIG. 14: Range Day 2, Shot 7, Null Test

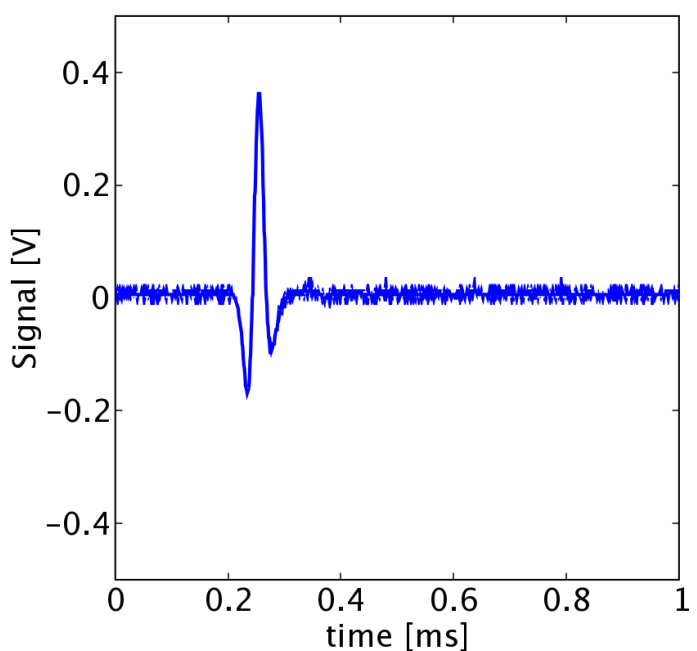

a) Signal from Magnetic Donut 1

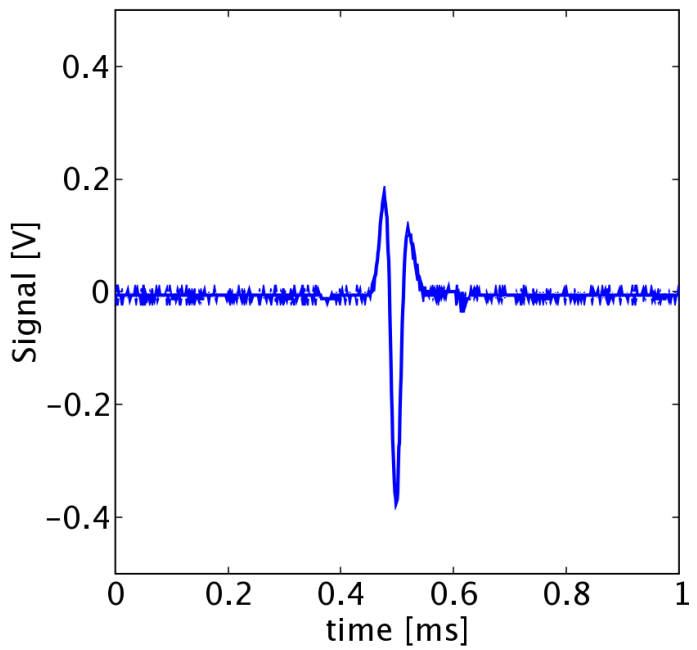

c) Signal from Magnetic Donut 3

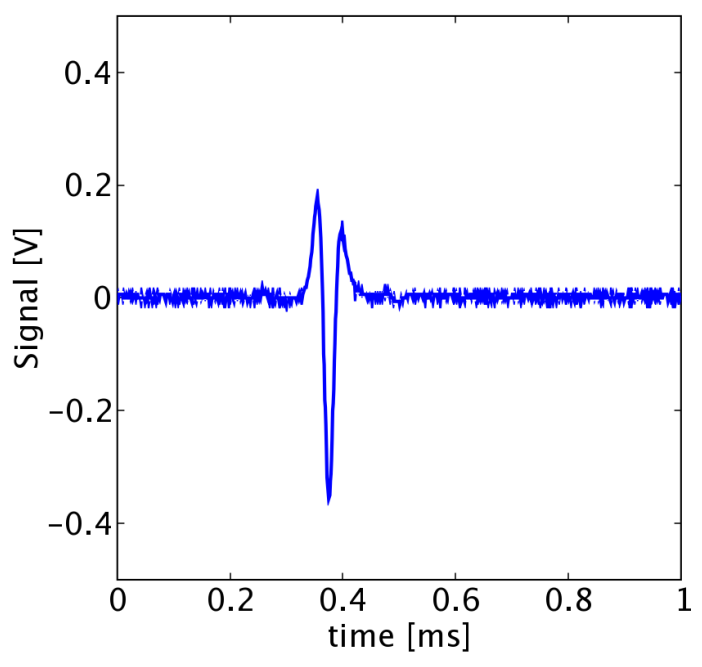

b) Signal from Magnetic Donut 2

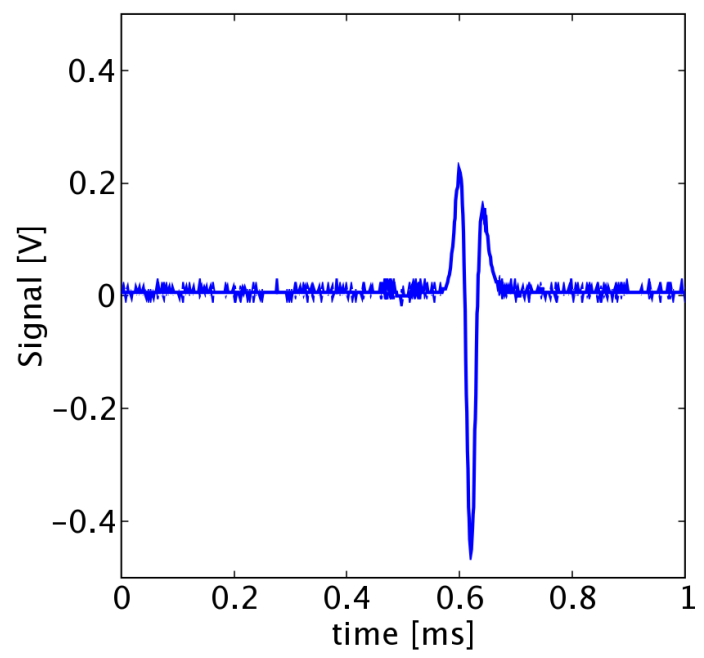

d) Signal from Magnetic Donut 4 
FIG. 15: Range Day 2, Shot 8, 10\# Foam

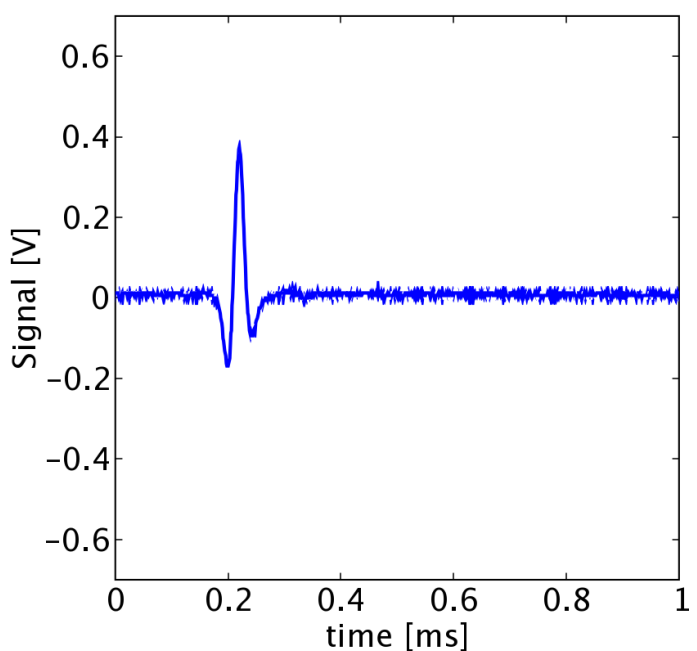

a) Signal from Magnetic Donut 1

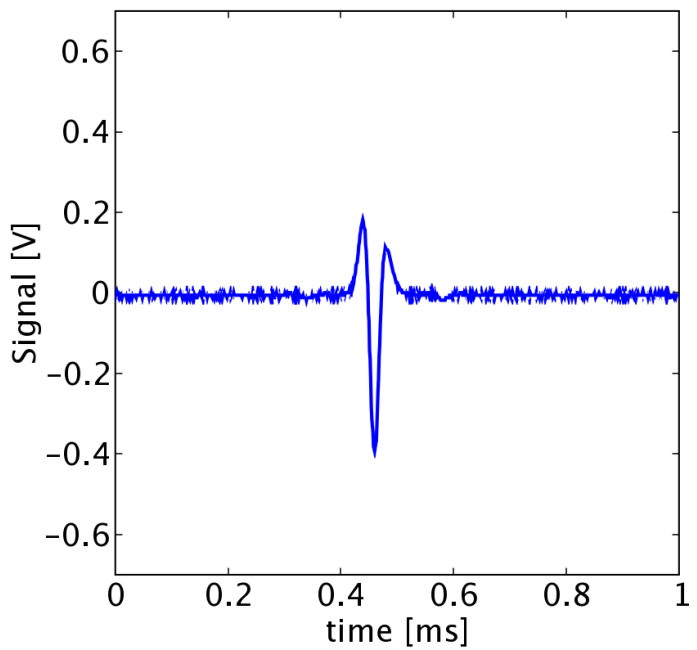

c) Signal from Magnetic Donut 3

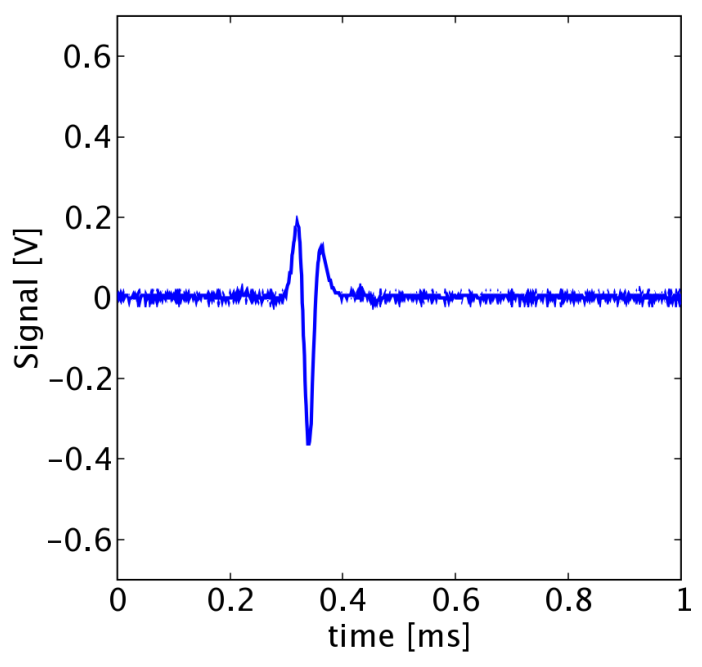

b) Signal from Magnetic Donut 2

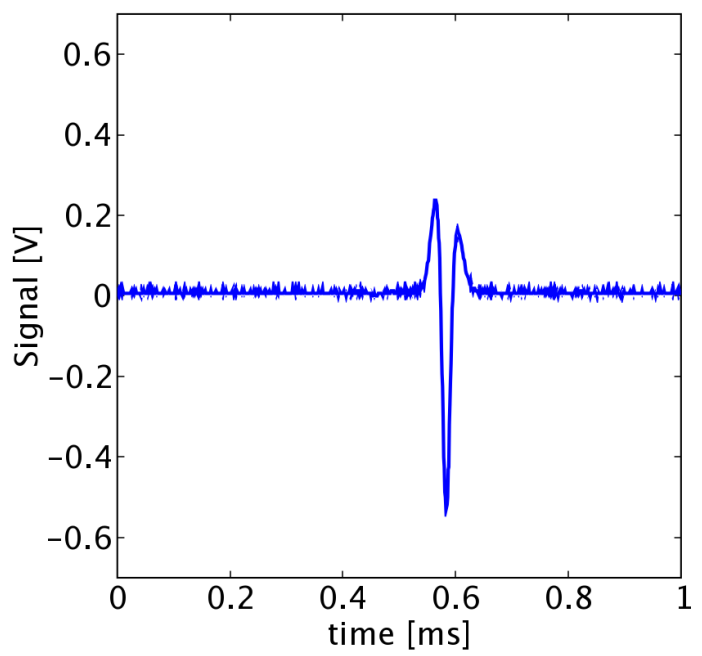

d) Signal from Magnetic Donut 4 
FIG. 16: Range Day 2, Shot 9, 20\# Foam

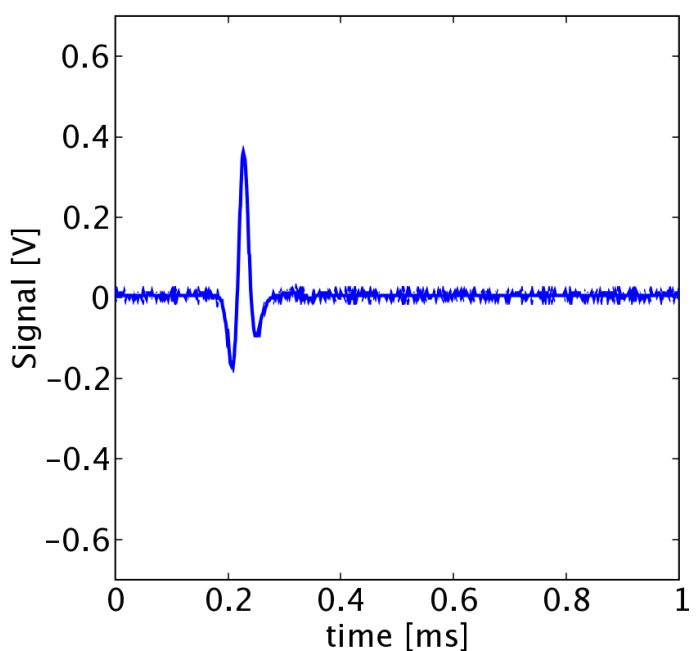

a) Signal from Magnetic Donut 1

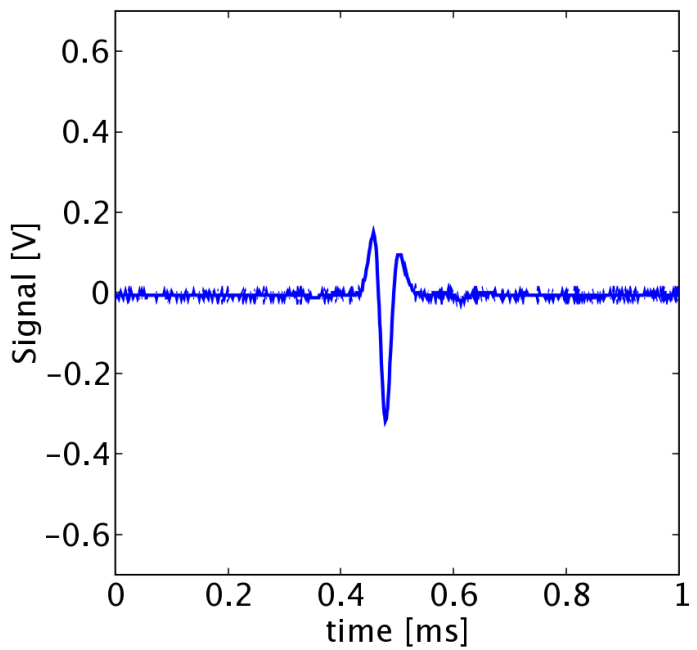

c) Signal from Magnetic Donut 3

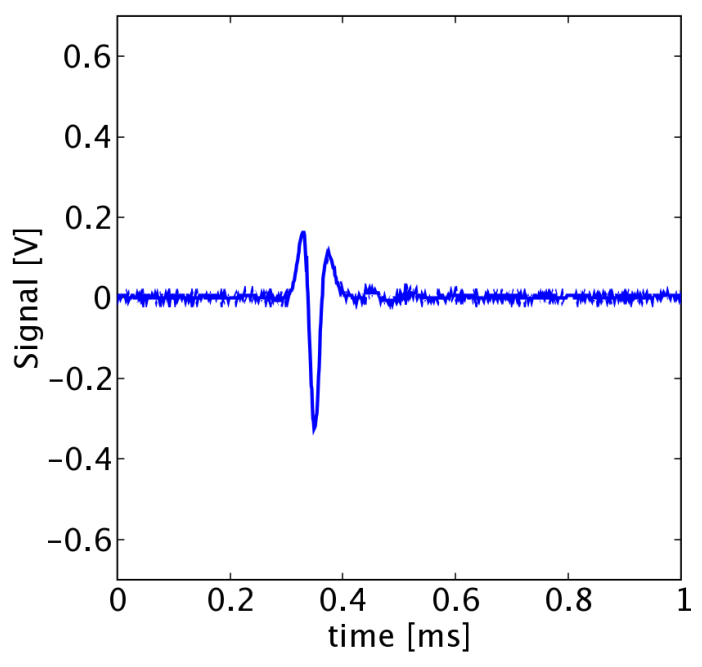

b) Signal from Magnetic Donut 2

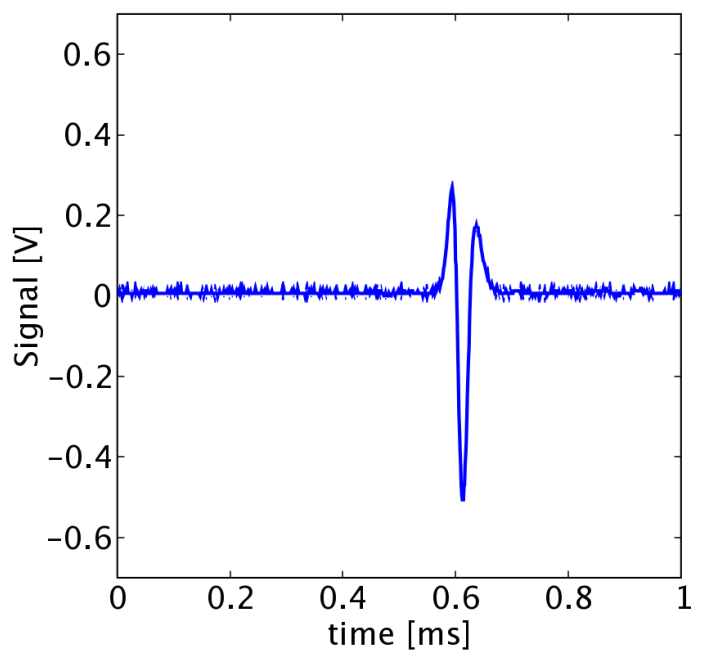

d) Signal from Magnetic Donut 4 
FIG. 17: Range Day 2, Shot 10, 20\# Foam

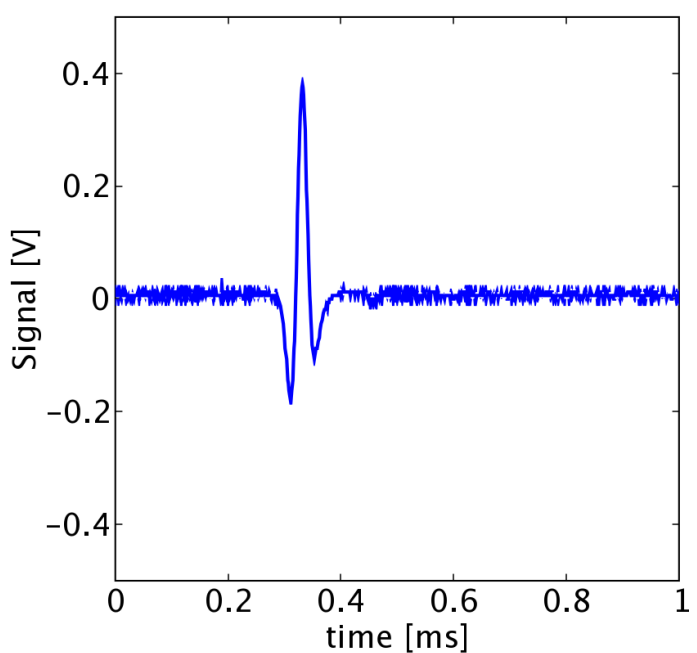

a) Signal from Magnetic Donut 1

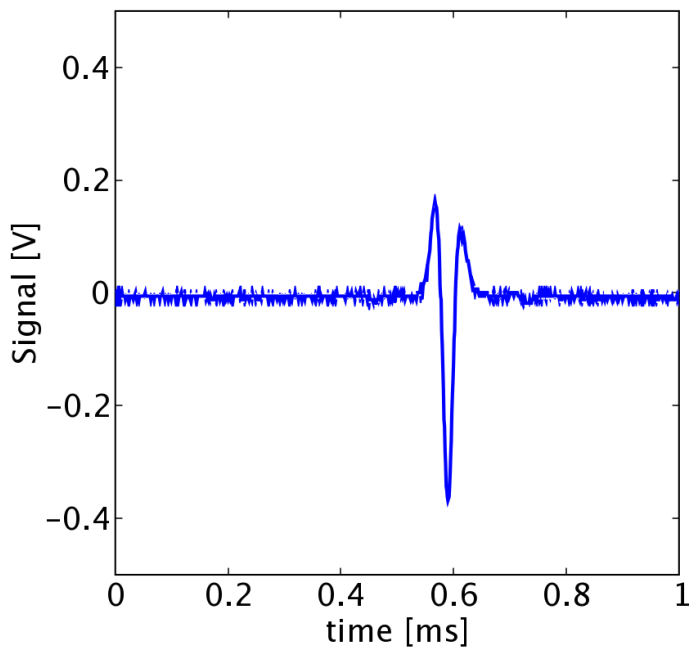

c) Signal from Magnetic Donut 3

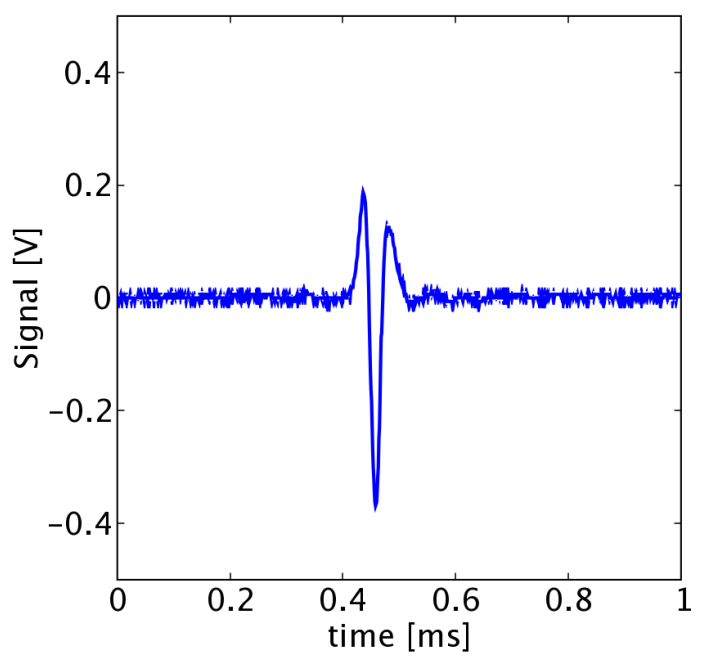

b) Signal from Magnetic Donut 2

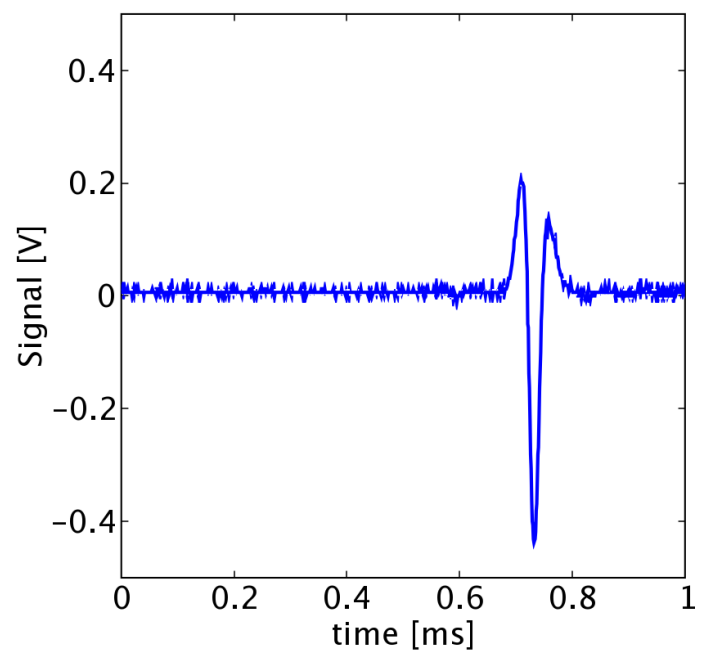

d) Signal from Magnetic Donut 4 
FIG. 18: Range Day 2, Shot 11, 20\# Foam

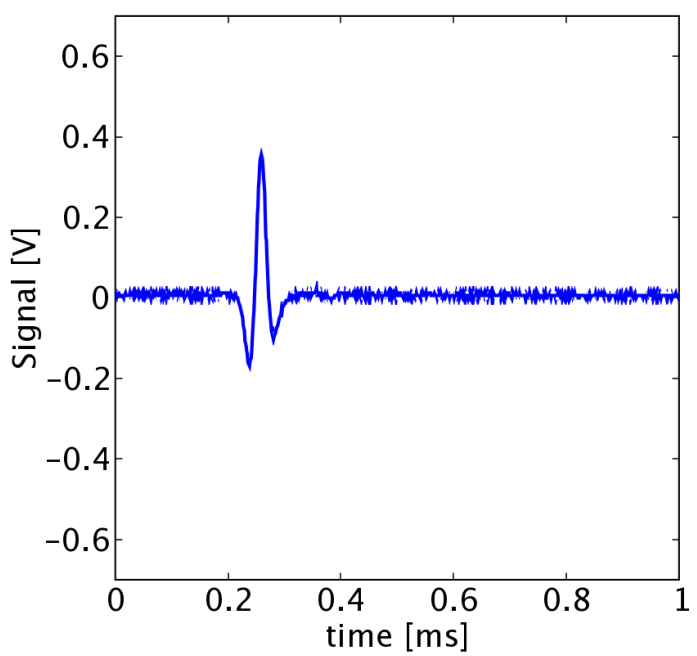

a) Signal from Magnetic Donut 1

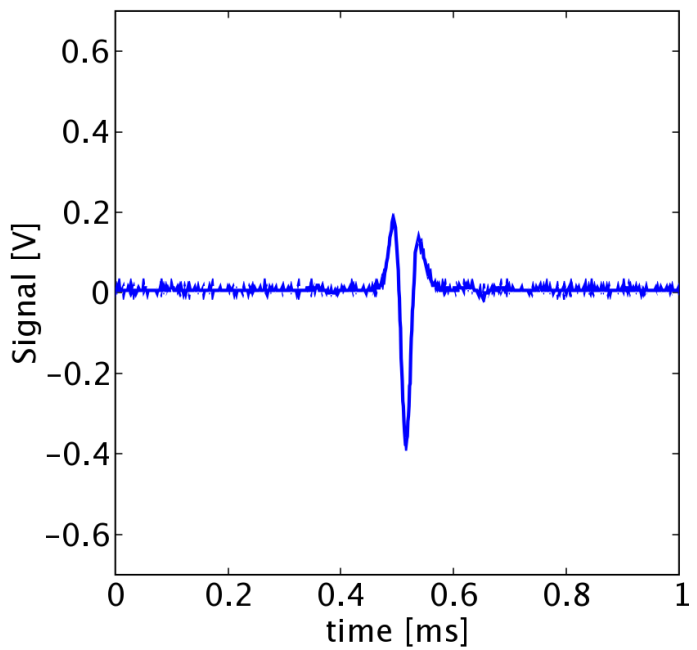

c) Signal from Magnetic Donut 3

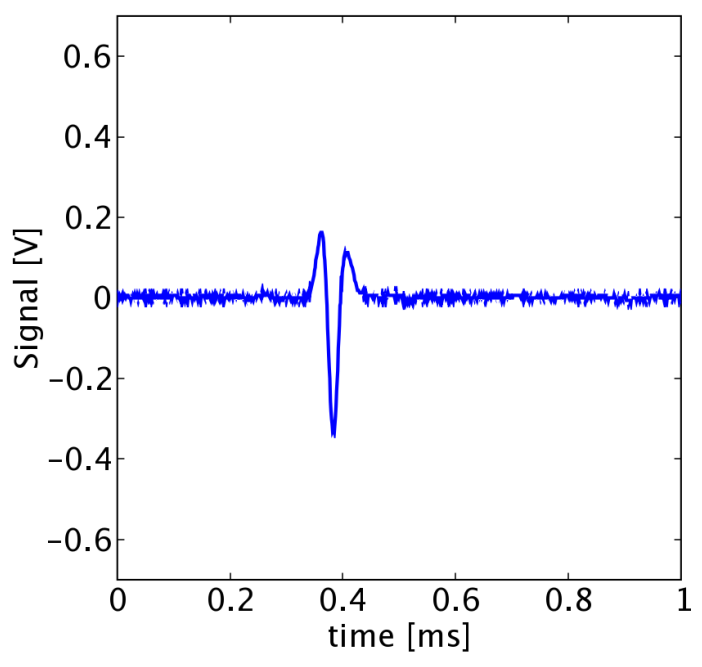

b) Signal from Magnetic Donut 2

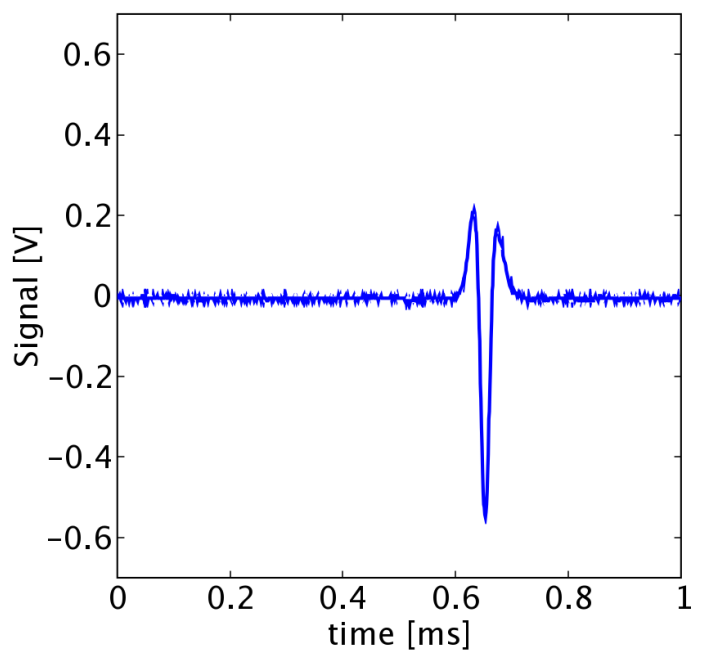

d) Signal from Magnetic Donut 4 
FIG. 19: Range Day 2, Shot 12, 20\# Foam

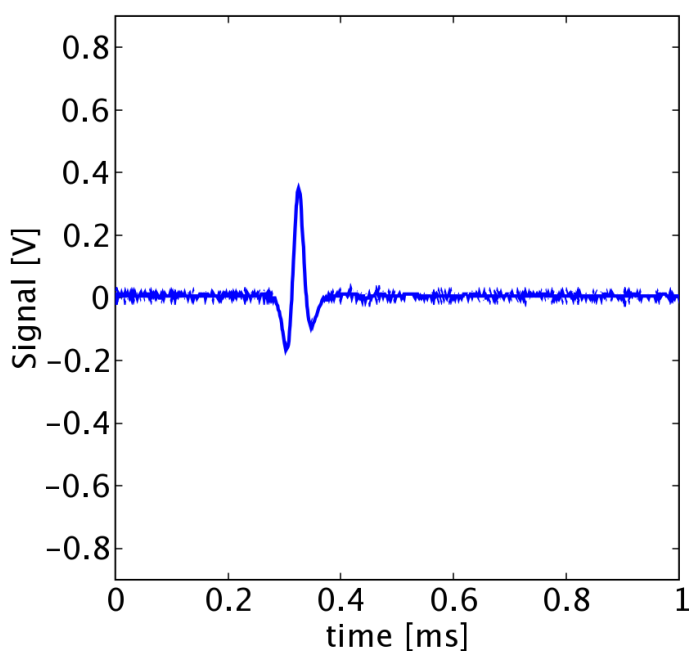

a) Signal from Magnetic Donut 1

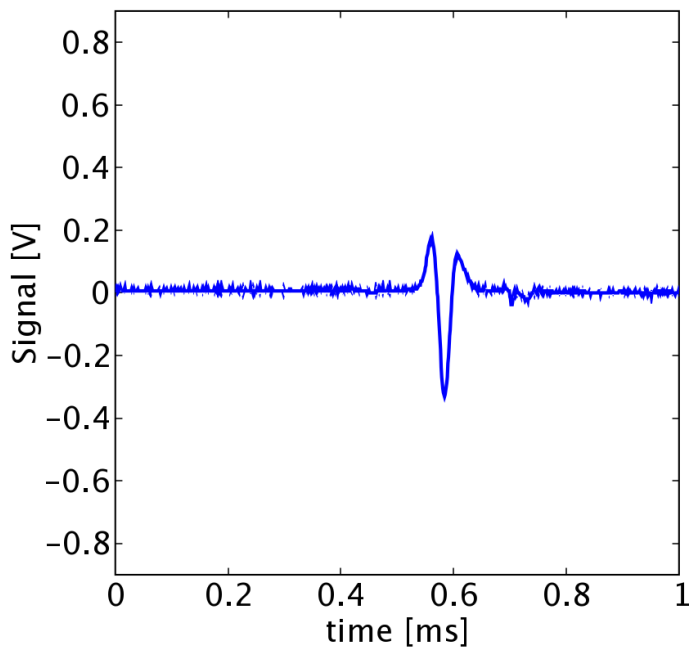

c) Signal from Magnetic Donut 3

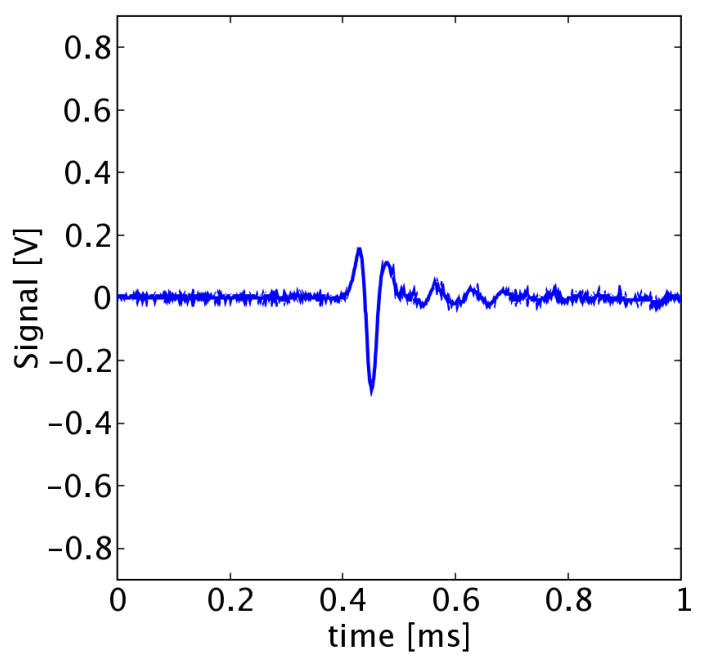

b) Signal from Magnetic Donut 2

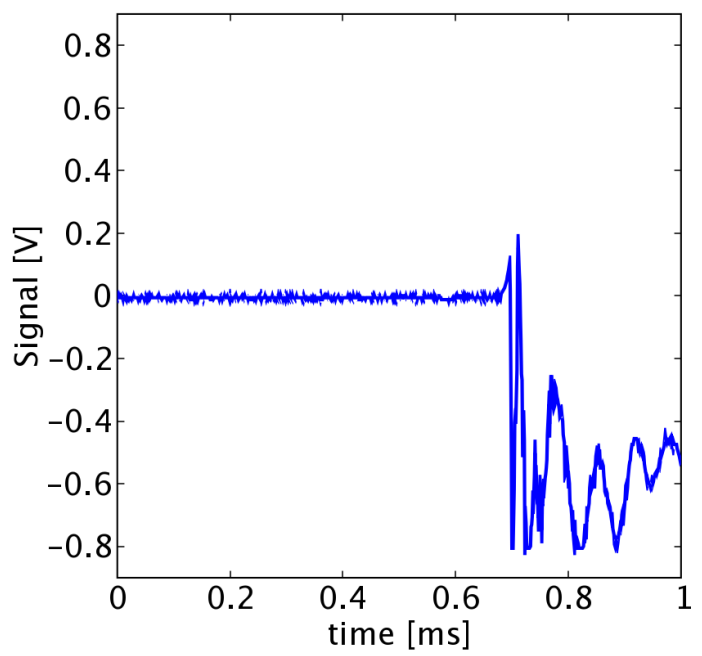

d) Signal from Magnetic Donut 4 
FIG. 20: Range Day 2, Shot 13, Null Test

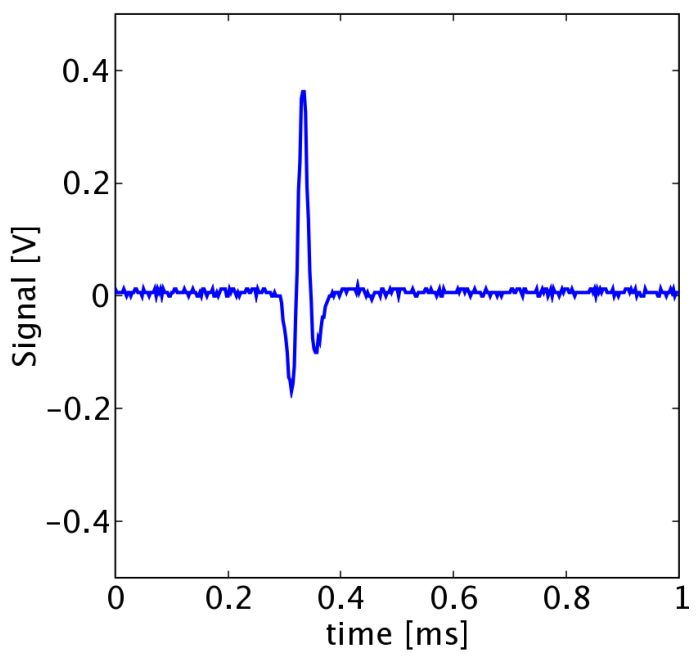

a) Signal from Magnetic Donut 1

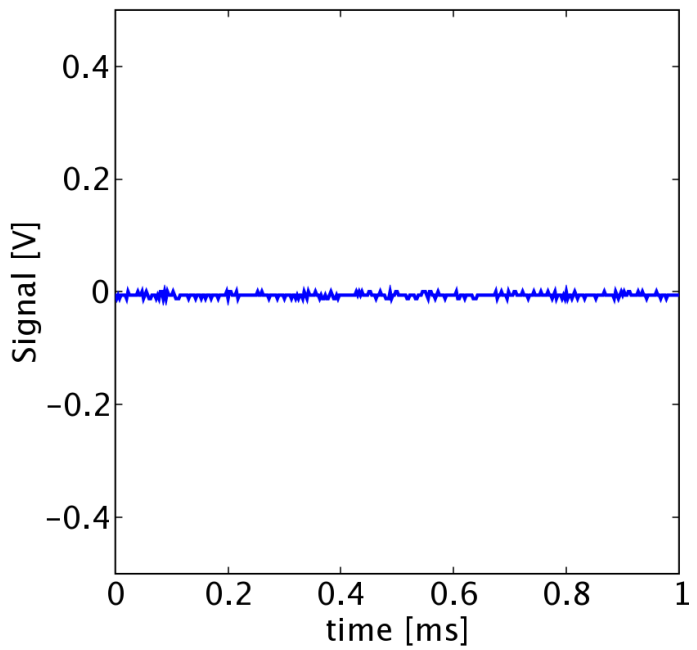

c) Signal from Magnetic Donut 3

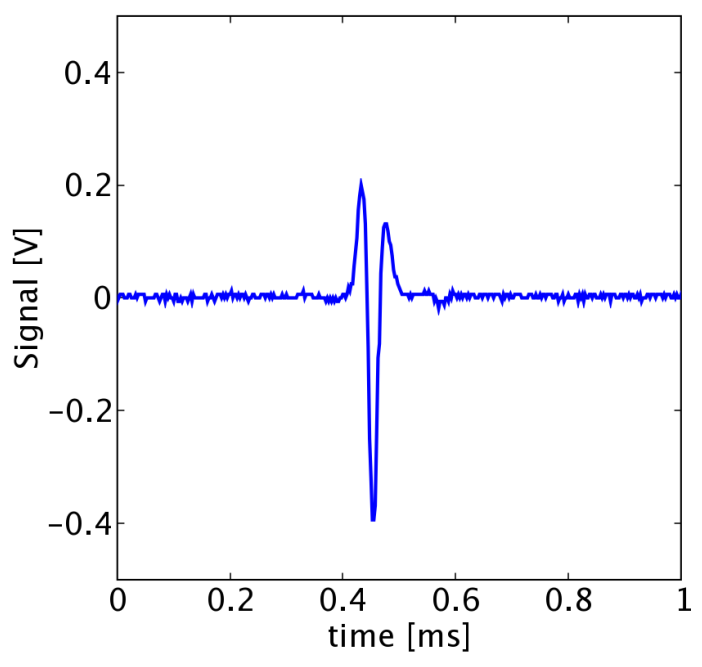

b) Signal from Magnetic Donut 2

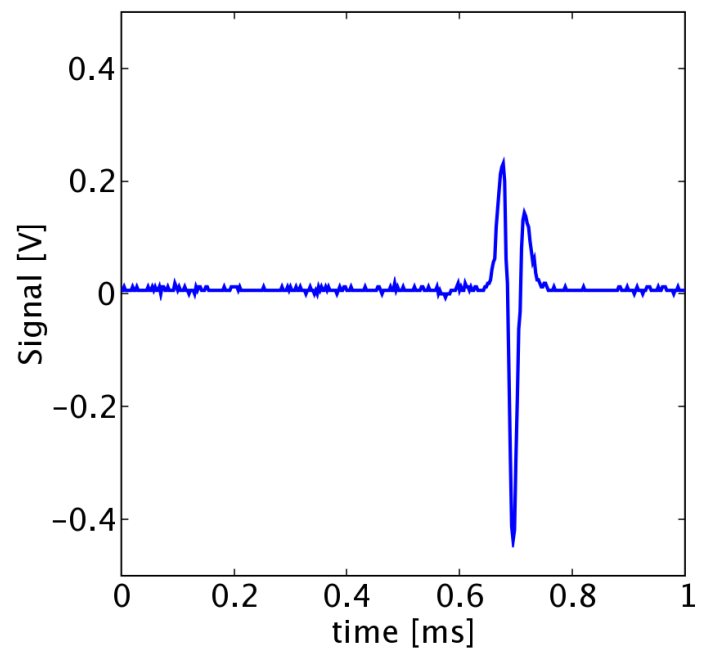

d) Signal from Magnetic Donut 4 
FIG. 21: Range Day 2, Shot 14, Null Test

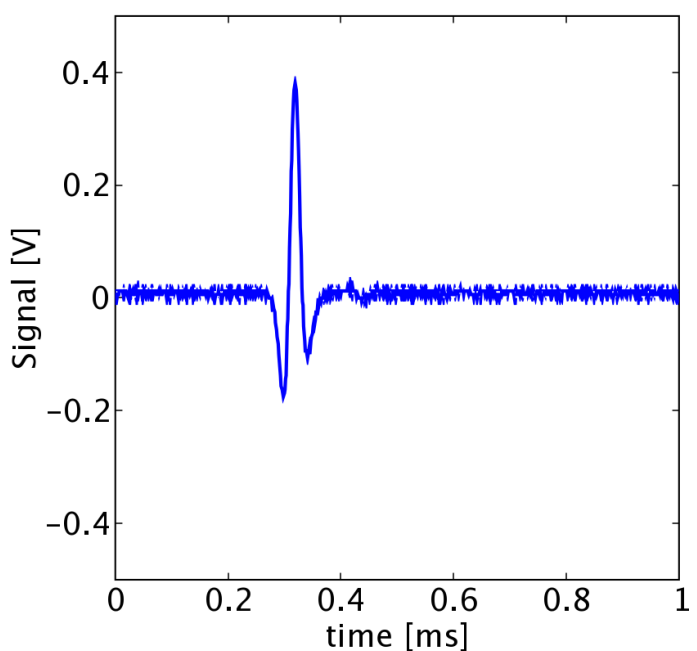

a) Signal from Magnetic Donut 1

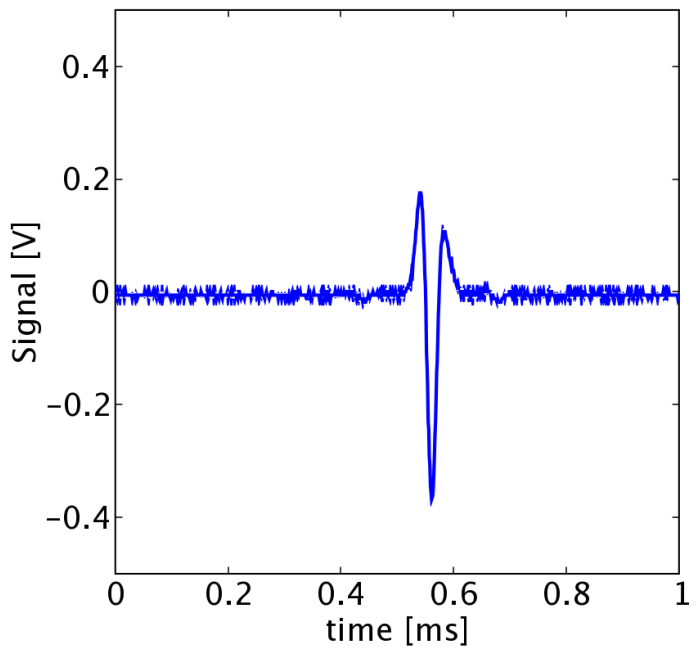

c) Signal from Magnetic Donut 3

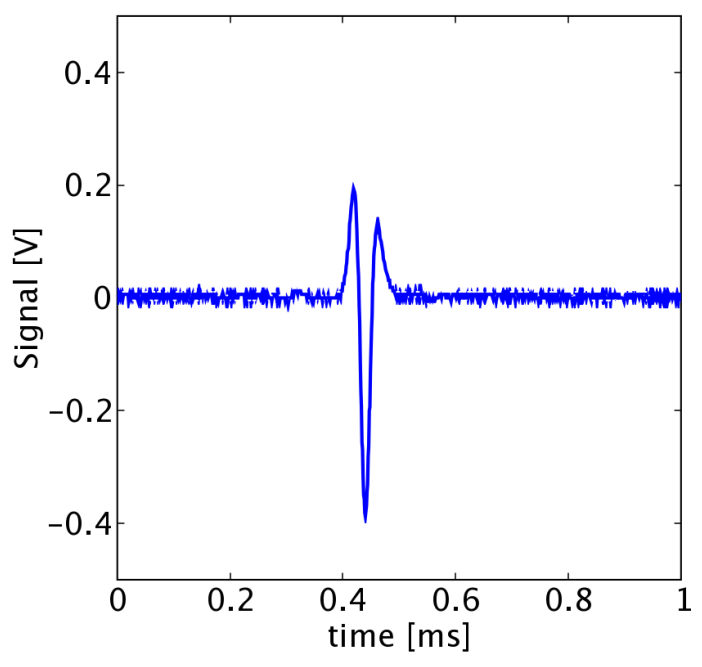

b) Signal from Magnetic Donut 2

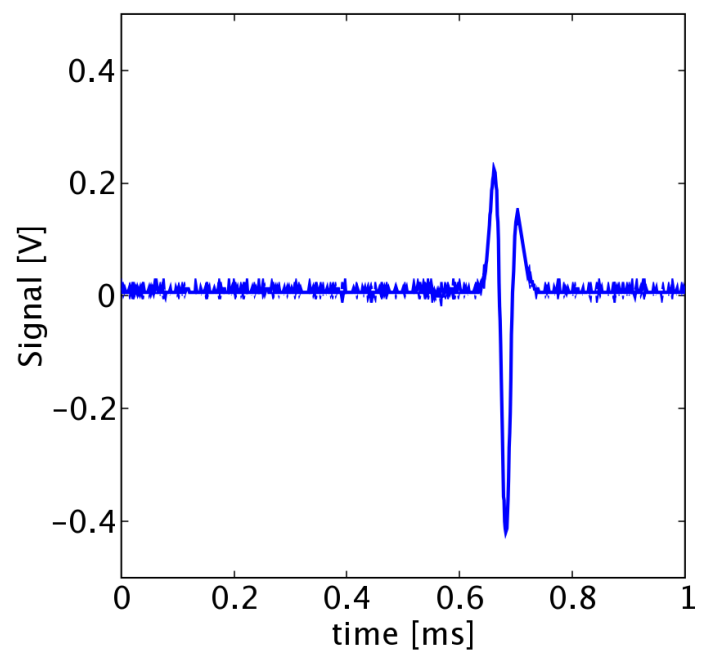

d) Signal from Magnetic Donut 4 
TABLE V: Range Day 2: Drag coefficients $c_{d}$ for shots with 10\# and 20\# foam targets. Drag coefficients were calculated using Eq. 8 with a projectile thickness (density) of $T=0.019[\mathbf{m}]\left(\rho=11.0\left[\mathrm{~g} / \mathbf{c m}^{3}\right]\right)$, foam target properties from Table $\mathbf{I}$, foam penetration distances from Table VI, and projectile velocities from Table VII.

\begin{tabular}{|c|c|c|c|c|c|c|c|c|}
\hline \multirow[b]{2}{*}{ donuts $\mathrm{A}$} & \multirow[b]{2}{*}{ donuts B } & \multicolumn{3}{|c|}{ 10\# Foam } & \multicolumn{4}{|c|}{ 20\# Foam } \\
\hline & & 5 & 6 & 8 & 9 & 10 & 11 & 12 \\
\hline $1-2$ & $2-3$ & 0.0000 & 0.0000 & 0.2117 & 0.7061 & 0.4926 & 0.4964 & 0.6897 \\
\hline $2-3$ & $3-4$ & 0.0000 & 0.0000 & 0.4183 & 0.3866 & 0.9315 & 0.5713 & 0.0000 \\
\hline $1-2$ & $3-4$ & 0.0000 & 0.0000 & 0.3150 & 0.5463 & 0.7121 & 0.5338 & 0.0000 \\
\hline
\end{tabular}

TABLE VI: Range Day 2: Projectile position $x$ and time coordinates $t$. Subscripts refer to shot numbers. Data assumes the Magnetic Donuts are spaced exactly 4 [in] apart and the maximum absolute value of the signal is used to assign a specific time to each detector.

\begin{tabular}{|c|c|c|c|c|c|c|c|c|}
\hline donut & $x[\mathrm{~m}]$ & $t_{1}[\mathrm{~s}]$ & $t_{2}[\mathrm{~s}]$ & $t_{3}[\mathrm{~s}]$ & $t_{4}[\mathrm{~s}]$ & $t_{5}[\mathrm{~s}]$ & $t_{6}[\mathrm{~s}]$ & $t_{7}[\mathrm{~s}]$ \\
\hline 1 & 0.0000 & $4.4800 \mathrm{e}-04$ & $3.5700 \mathrm{e}-04$ & $3.6100 \mathrm{e}-04$ & $2.3100 \mathrm{e}-04$ & $2.7550 \mathrm{e}-04$ & $1.6700 \mathrm{e}-04$ & $2.5400 \mathrm{e}-04$ \\
\hline 2 & 0.1016 & $5.7600 \mathrm{e}-04$ & $4.8100 \mathrm{e}-04$ & $4.8600 \mathrm{e}-04$ & $3.5150 \mathrm{e}-04$ & $4.0550 \mathrm{e}-04$ & $2.8800 \mathrm{e}-04$ & $3.7600 \mathrm{e}-04$ \\
\hline 3 & 0.2032 & $7.0100 \mathrm{e}-04$ & $0.0000 \mathrm{e}+00$ & $6.0950 \mathrm{e}-04$ & $4.7250 \mathrm{e}-04$ & $0.0000 \mathrm{e}+00$ & $0.0000 \mathrm{e}+00$ & $4.9900 \mathrm{e}-04$ \\
\hline 4 & 0.3048 & $8.2500 \mathrm{e}-04$ & $7.2600 \mathrm{e}-04$ & $7.3200 \mathrm{e}-04$ & $5.8950 \mathrm{e}-04$ & $0.0000 \mathrm{e}+00$ & $5.3250 \mathrm{e}-04$ & $6.2000 \mathrm{e}-04$ \\
\hline donut & $x[\mathrm{~m}]$ & $t_{8}[\mathrm{~s}]$ & $t_{9}[\mathrm{~s}]$ & $t_{10}[\mathrm{~s}]$ & $t_{11}[\mathrm{~s}]$ & $t_{12}[\mathrm{~s}]$ & $t_{13}[\mathrm{~s}]$ & $t_{14}[\mathrm{~s}]$ \\
\hline 1 & 0.0000 & $2.2000 \mathrm{e}-04$ & $2.2700 \mathrm{e}-04$ & $3.3200 \mathrm{e}-04$ & $2.5900 \mathrm{e}-04$ & $3.2500 \mathrm{e}-04$ & $3.3250 \mathrm{e}-04$ & $3.1900 \mathrm{e}-04$ \\
\hline 2 & 0.1016 & $3.4000 \mathrm{e}-04$ & $3.5000 \mathrm{e}-04$ & $4.5900 \mathrm{e}-04$ & $3.8500 \mathrm{e}-04$ & $4.5100 \mathrm{e}-04$ & $4.5500 \mathrm{e}-04$ & $4.4100 \mathrm{e}-04$ \\
\hline 3 & 0.2032 & $4.6100 \mathrm{e}-04$ & $4.8000 \mathrm{e}-04$ & $5.9100 \mathrm{e}-04$ & $5.1600 \mathrm{e}-04$ & $5.8400 \mathrm{e}-04$ & $0.0000 \mathrm{e}+00$ & $5.6200 \mathrm{e}-04$ \\
\hline 4 & 0.3048 & $5.8400 \mathrm{e}-04$ & $6.1400 \mathrm{e}-04$ & $7.3300 \mathrm{e}-04$ & $6.5300 \mathrm{e}-04$ & $0.0000 \mathrm{e}+00$ & $6.9500 \mathrm{e}-04$ & $6.8300 \mathrm{e}-04$ \\
\hline
\end{tabular}

TABLE VII: Range Day 2: Projectile velocities $v$ and initial chronograph velocities. Subscripts refer to shot numbers. Velocity is determined by $v_{i j}=\Delta x / \Delta t$, where $\Delta x=x_{j}-x_{i}\left(\Delta t=t_{j}-t_{i}\right)$ and the subscripts reference different Magnetic Donuts. Initial chronograph velocities are in the last row of each shot.

\begin{tabular}{ccccccccc}
\hline \hline donut A & donut B & $v_{1}[\mathrm{~m} / \mathrm{s}]$ & $v_{2}[\mathrm{~m} / \mathrm{s}]$ & $v_{3}[\mathrm{~m} / \mathrm{s}]$ & $v_{4}[\mathrm{~m} / \mathrm{s}]$ & $v_{5}[\mathrm{~m} / \mathrm{s}]$ & $v_{6}[\mathrm{~m} / \mathrm{s}]$ & $v_{7}[\mathrm{~m} / \mathrm{s}]$ \\
\hline 1 & 2 & 793.75 & 819.35 & 812.80 & 843.15 & 781.54 & 839.67 & 832.79 \\
1 & 3 & 803.16 & 000.00 & 817.71 & 841.41 & 000.00 & 000.00 & 829.39 \\
1 & 4 & 808.49 & 826.02 & 821.56 & 850.21 & 000.00 & 833.93 & 832.79 \\
2 & 3 & 812.80 & 000.00 & 822.67 & 839.67 & 000.00 & 000.00 & 826.02 \\
2 & 4 & 816.06 & 829.39 & 826.02 & 853.78 & 000.00 & 831.08 & 832.79 \\
3 & 4 & 819.35 & 000.00 & 829.39 & 868.38 & 000.00 & 000.00 & 839.67 \\
\multicolumn{2}{l}{ Chronograph } & 788.82 & 808.02 & 801.62 & 827.84 & 812.30 & 831.80 & 831.19 \\
\multicolumn{1}{l}{ donut A } & donut B & $v_{8}[\mathrm{~m} / \mathrm{s}]$ & $v_{9}[\mathrm{~m} / \mathrm{s}]$ & $v_{10}[\mathrm{~m} / \mathrm{s}]$ & $v_{11}[\mathrm{~m} / \mathrm{s}]$ & $v_{12}[\mathrm{~m} / \mathrm{s}]$ & $v_{13}[\mathrm{~m} / \mathrm{s}]$ & $v_{14}[\mathrm{~m} / \mathrm{s}]$ \\
\hline 1 & 2 & 846.67 & 826.02 & 800.00 & 806.35 & 806.35 & 829.39 & 832.79 \\
1 & 3 & 843.15 & 803.16 & 784.56 & 790.66 & 784.56 & 000.00 & 836.21 \\
1 & 4 & 837.36 & 787.60 & 760.10 & 773.60 & 000.00 & 840.83 & 837.36 \\
2 & 3 & 839.67 & 781.54 & 769.70 & 775.57 & 763.91 & 000.00 & 839.67 \\
2 & 4 & 832.79 & 769.70 & 741.61 & 758.21 & 000.00 & 846.67 & 839.67 \\
3 & 4 & 826.02 & 758.21 & 715.49 & 741.61 & 000.00 & 000.00 & 839.67 \\
\multicolumn{2}{l}{ Chronograph } & 843.08 & 839.11 & 819.92 & 829.97 & 812.30 & 817.78 & 820.22 \\
\hline \hline
\end{tabular}


FIG. 22: Krispy Kreme Shot 1392 (25 March 2005): The projectile was a 19.05 [mm] diameter Ta disk with thickness $2.67[\mathrm{~mm}]$, there was no foam target, and 8 Magnetic Donuts were fielded. X-ray velocity $=1.07[\mathrm{~km} / \mathrm{s}]$.

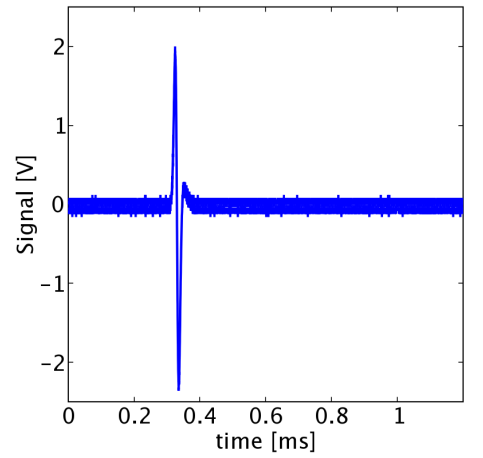

a) Signal from Magnetic Donut 1

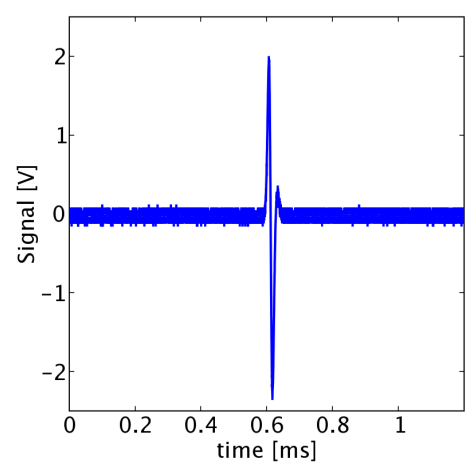

d) Signal from Magnetic Donut 4

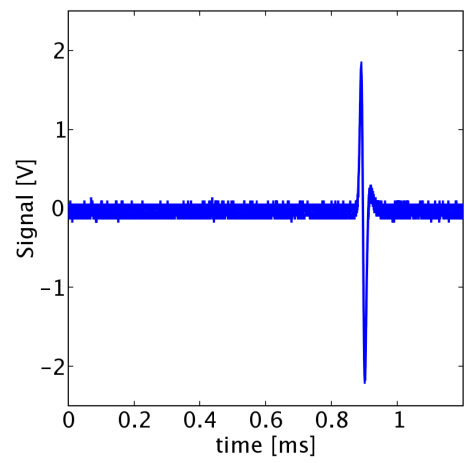

g) Signal from Magnetic Donut 7

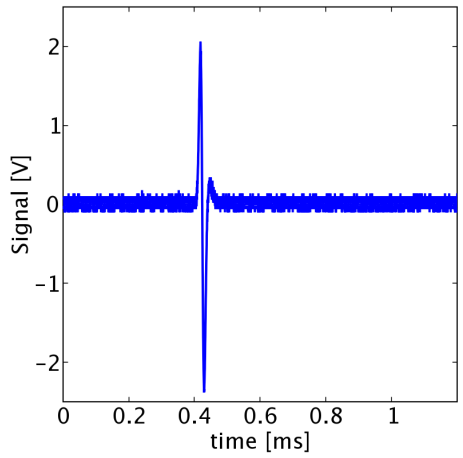

b) Signal from Magnetic Donut 2

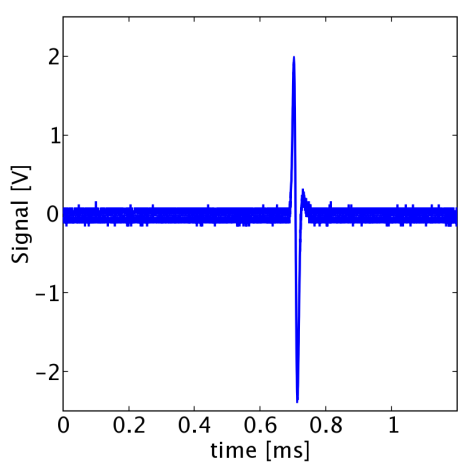

e) Signal from Magnetic Donut 5

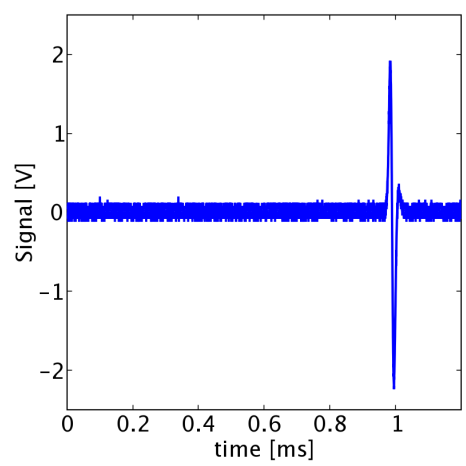

h) Signal from Magnetic Donut 8

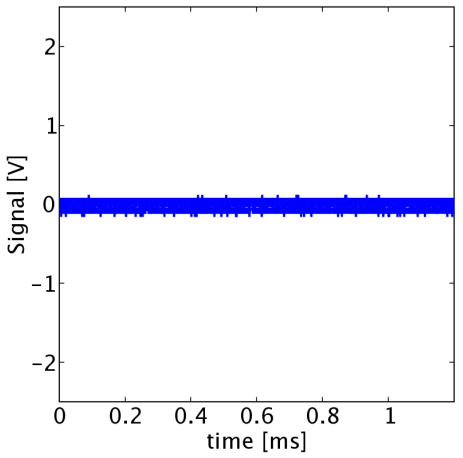

c) Signal from Magnetic Donut 3

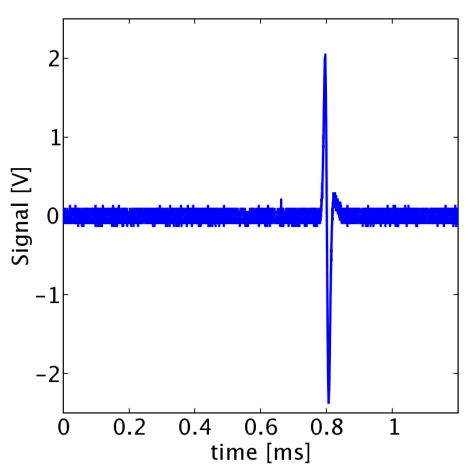

f) Signal from Magnetic Donut 6

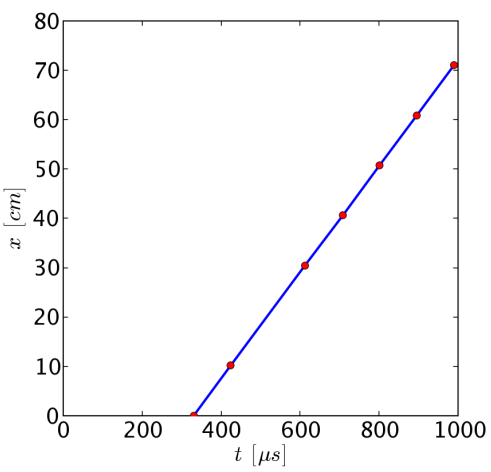

i) Fan plot 
FIG. 23: Krispy Kreme Shot 1393 (29 March 2005): The projectile was a 19.05 [mm] diameter Ta disk with thickness $2.67[\mathrm{~mm}]$, there was no foam target, and 8 Magnetic Donuts were fielded. X-ray velocity $=0.96[\mathrm{~km} / \mathrm{s}]$.

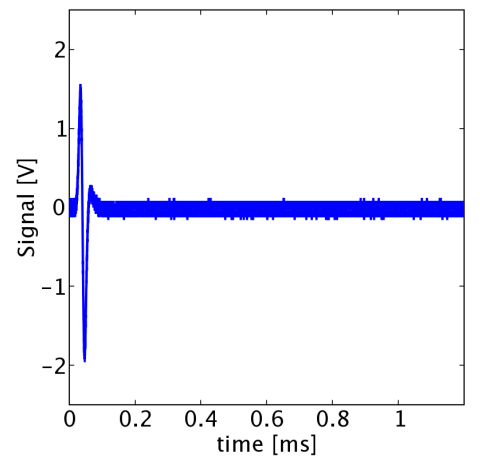

a) Signal from Magnetic Donut 1

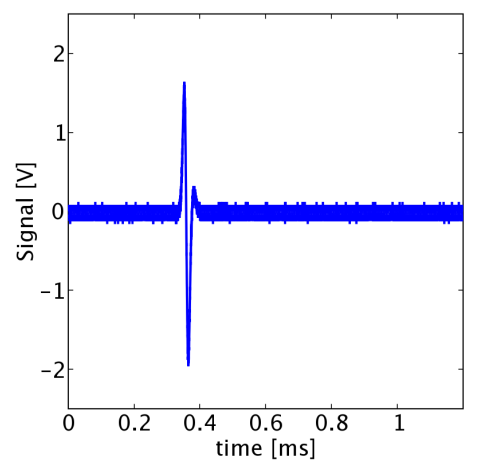

d) Signal from Magnetic Donut 4

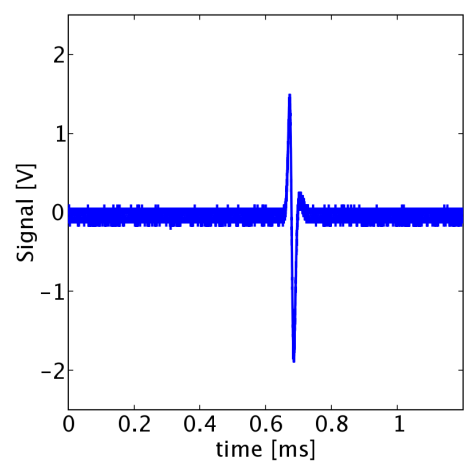

g) Signal from Magnetic Donut 7

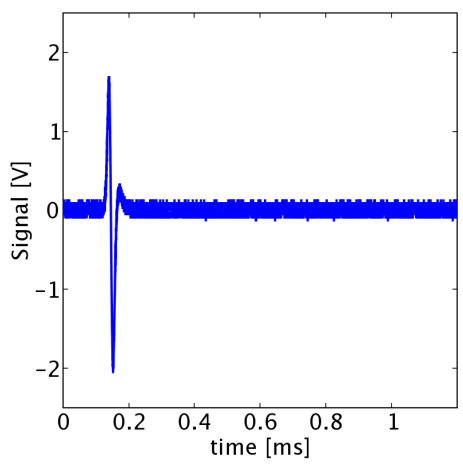

b) Signal from Magnetic Donut 2

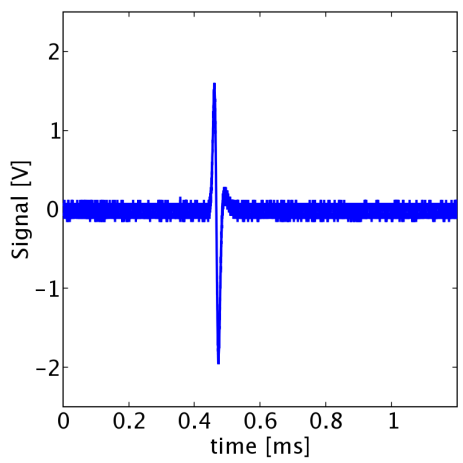

e) Signal from Magnetic Donut 5

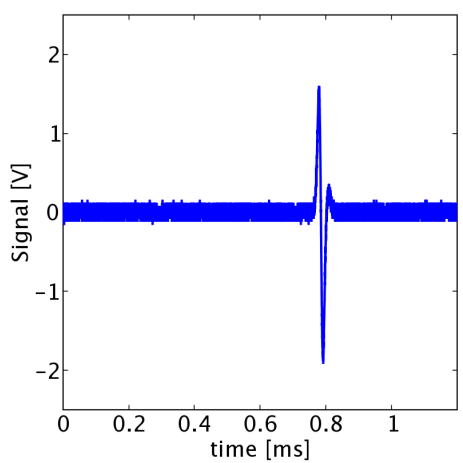

h) Signal from Magnetic Donut 8

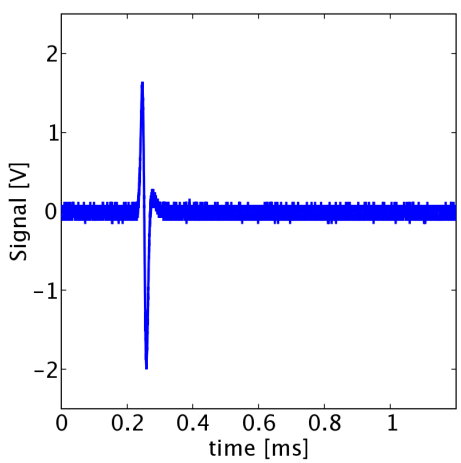

c) Signal from Magnetic Donut 3

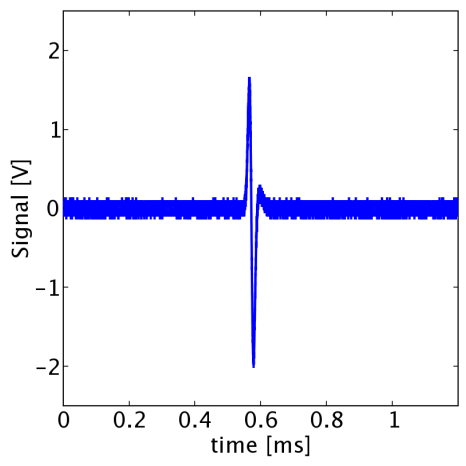

f) Signal from Magnetic Donut 6

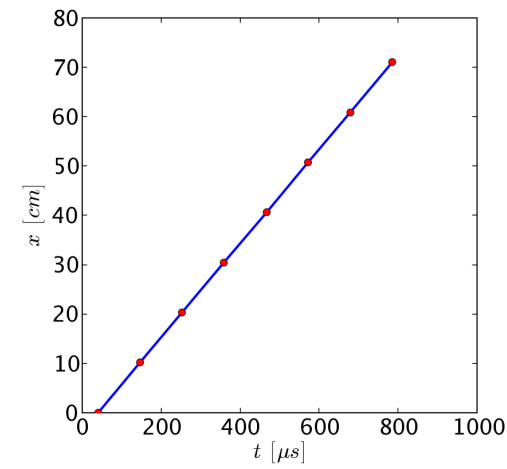

i) Fan plot 
FIG. 24: Krispy Kreme Shot 1394 (30 March 2005): The projectile was a Ta block with square side length 3.0 [mm] and thickness 2.67 [mm], there was a 10\# foam target placed between Magnetic Donut locations 3-6, and 6 Magnetic Donuts were fielded at locations 2-7.

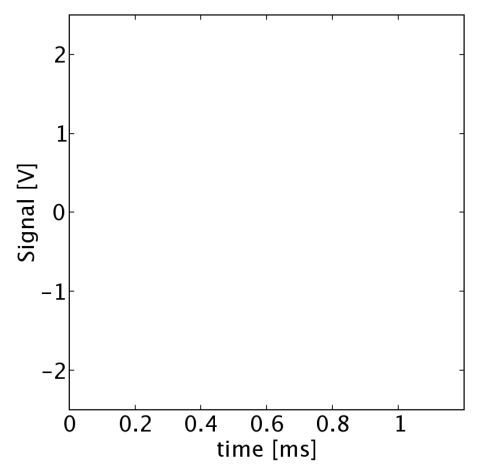

a) Signal from Magnetic Donut 1

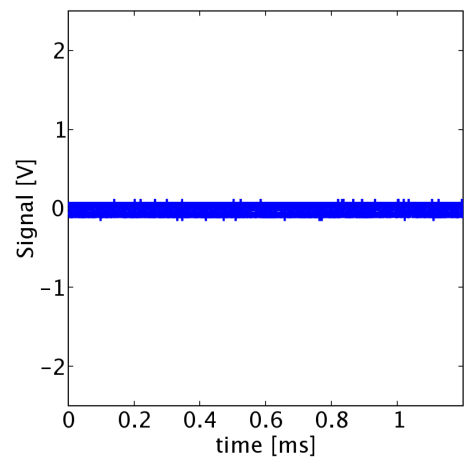

d) Signal from Magnetic Donut 4

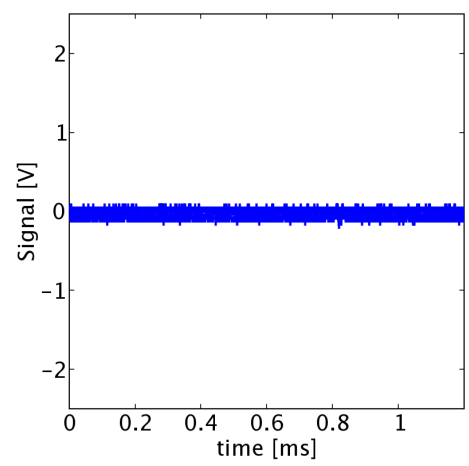

g) Signal from Magnetic Donut 7

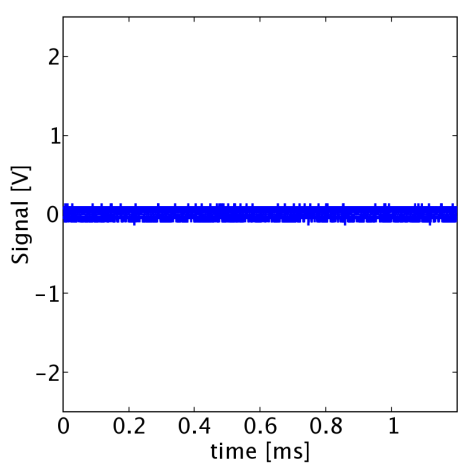

b) Signal from Magnetic Donut 2

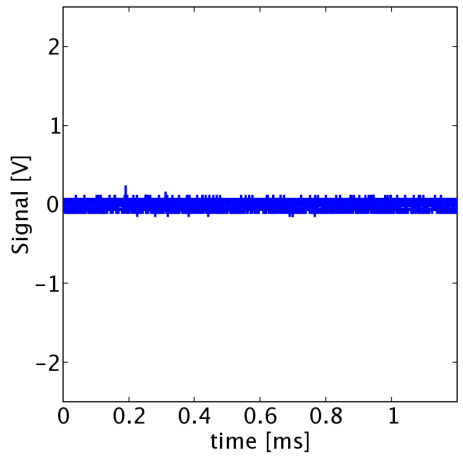

e) Signal from Magnetic Donut 5

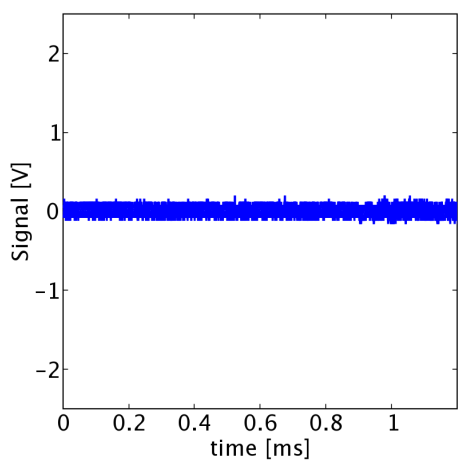

h) Signal from Magnetic Donut 8

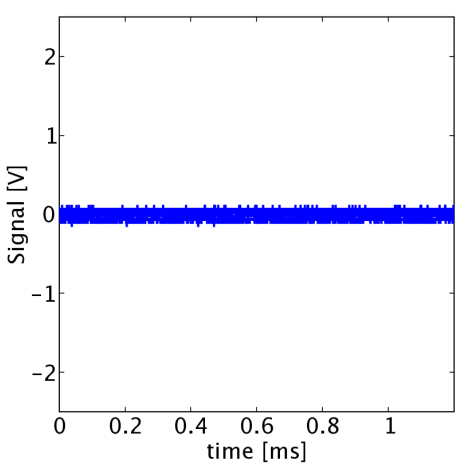

c) Signal from Magnetic Donut 3

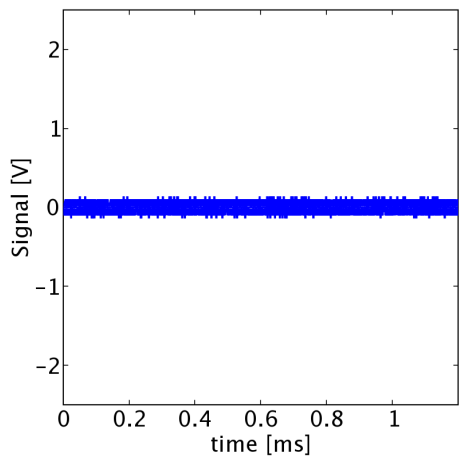

f) Signal from Magnetic Donut 6

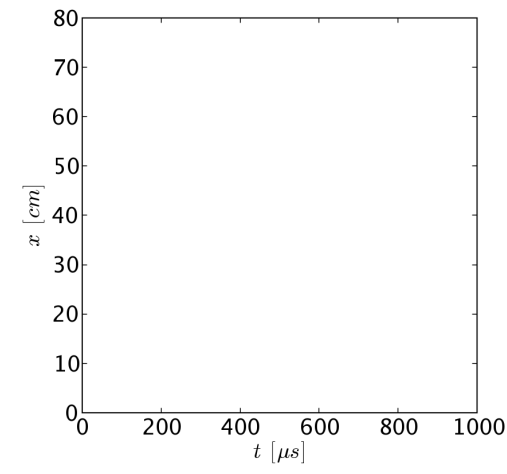

i) Fan plot 
FIG. 25: Krispy Kreme Shot 1395 (30 March 2005): The projectile was a 9.0 [mm] diameter Ta disk with thickness $2.67[\mathrm{~mm}]$, there was no foam target, and 8 Magnetic Donuts were fielded. X-ray velocity $=1.12[\mathrm{~km} / \mathrm{s}]$.

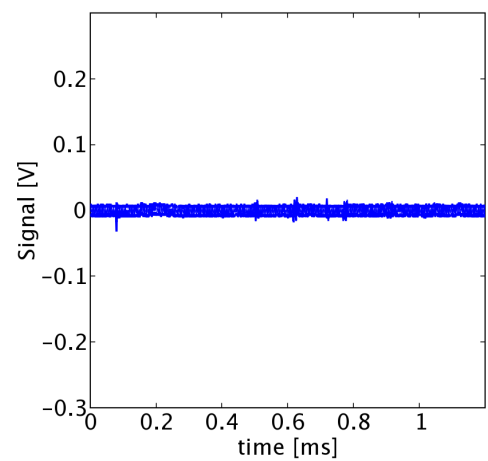

a) Signal from Magnetic Donut 1

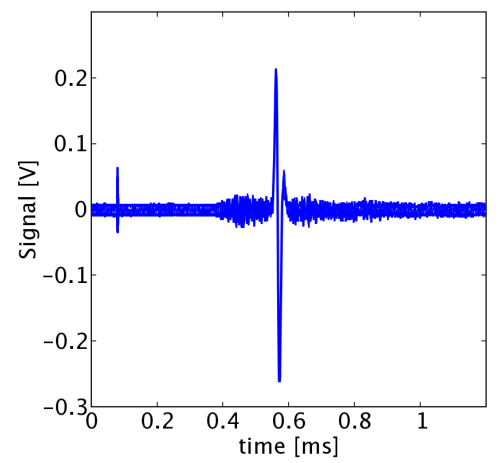

d) Signal from Magnetic Donut 4

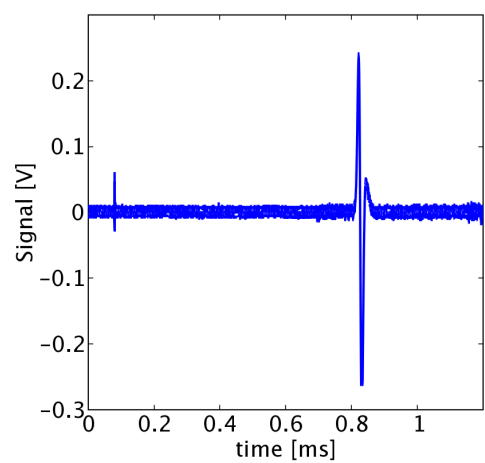

g) Signal from Magnetic Donut 7

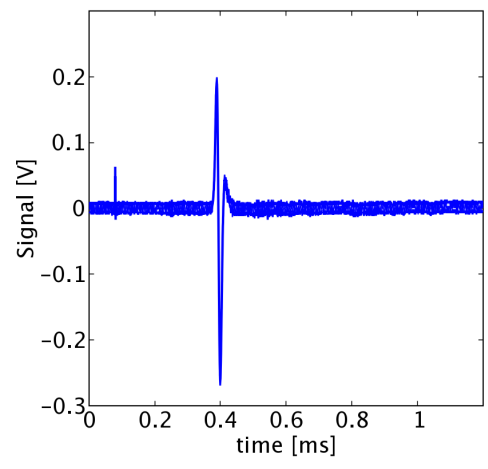

b) Signal from Magnetic Donut 2

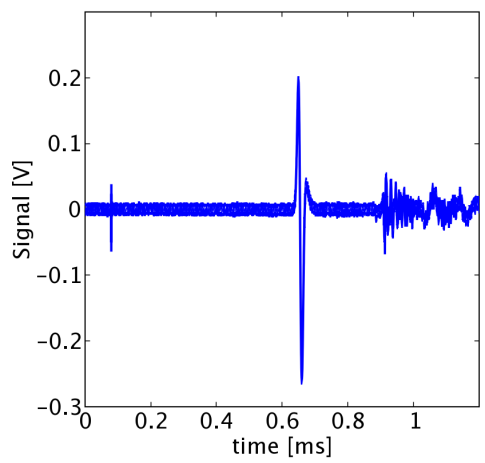

e) Signal from Magnetic Donut 5

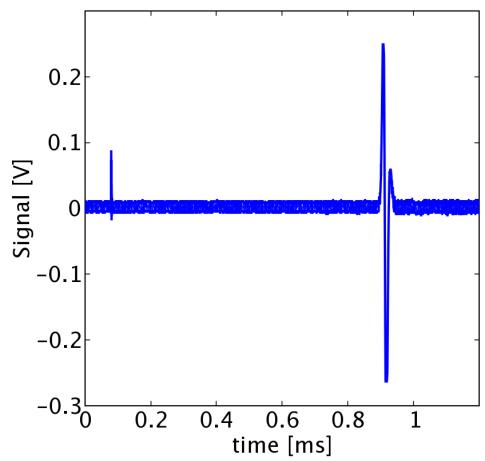

h) Signal from Magnetic Donut 8

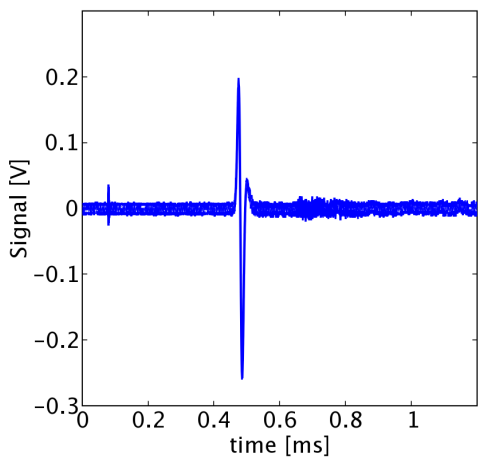

c) Signal from Magnetic Donut 3

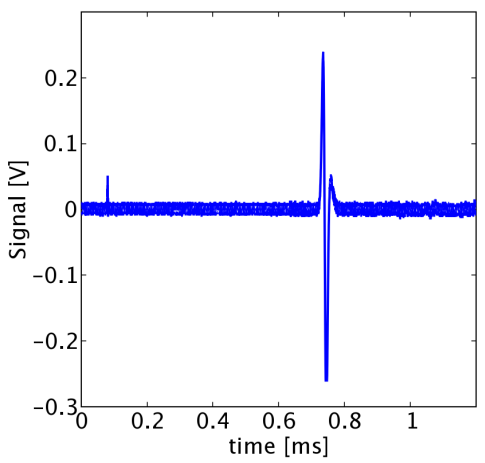

f) Signal from Magnetic Donut 6

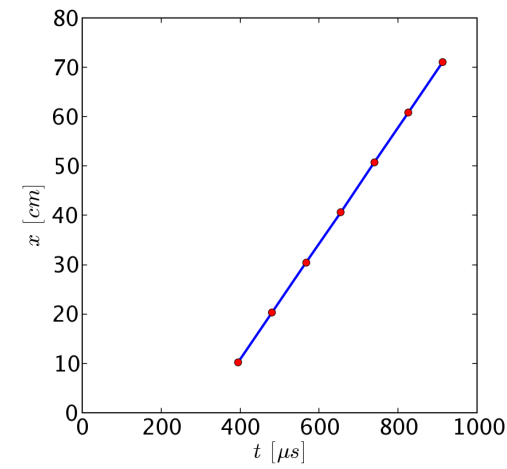

i) Fan plot 
FIG. 26: Krispy Kreme Shot 1396 (31 March 2005): The projectile was a Ta block with square side length 5.0 [mm] and thickness 2.67 [mm], there was a 10\# foam target placed between Magnetic Donut locations 3-6, and 6 Magnetic Donuts were fielded at locations $2-7$. X-ray velocity $=1.11[\mathrm{~km} / \mathrm{s}]$.

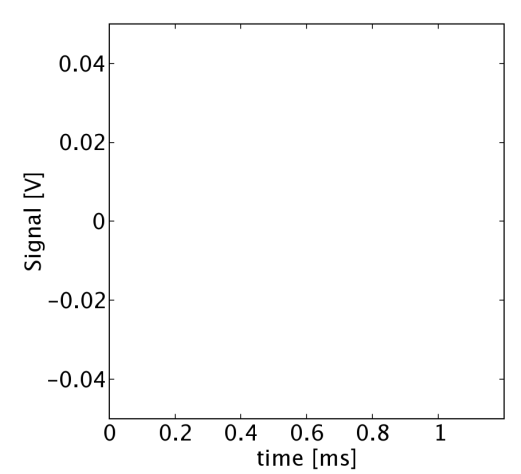

a) Signal from Magnetic Donut 1

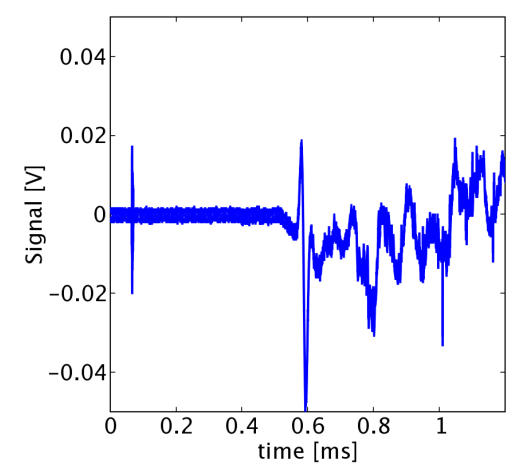

d) Signal from Magnetic Donut 4

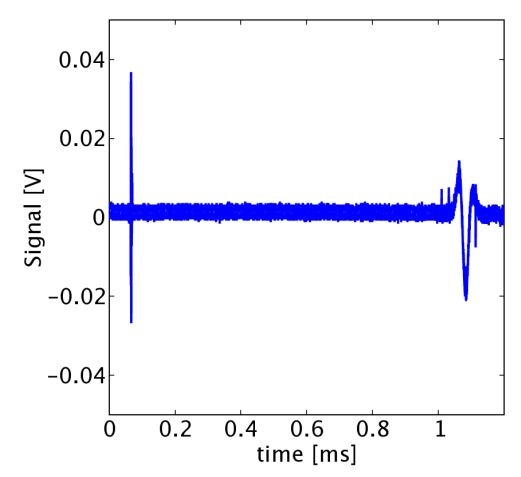

g) Signal from Magnetic Donut 7

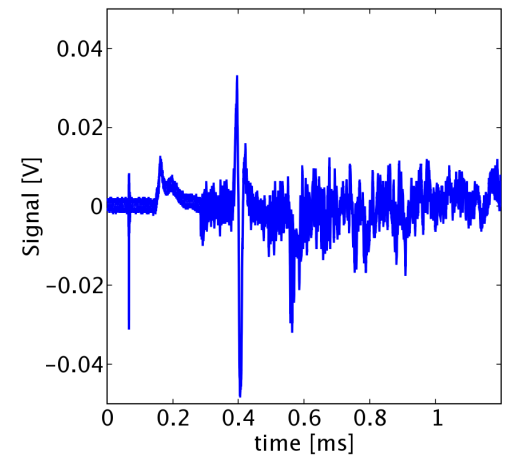

b) Signal from Magnetic Donut 2

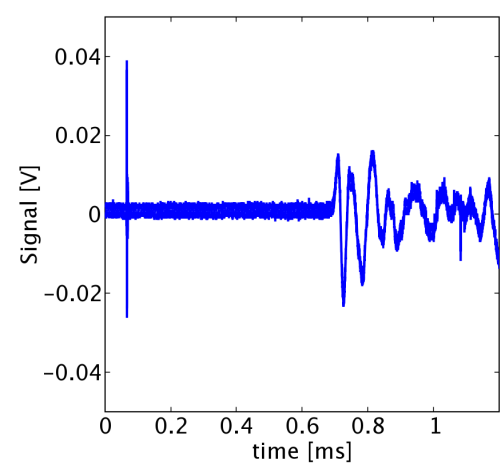

e) Signal from Magnetic Donut 5

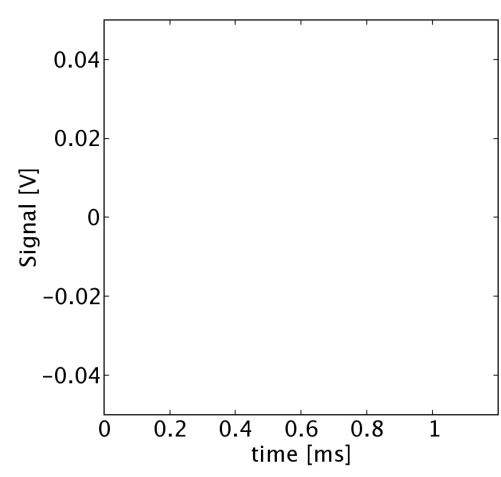

h) Signal from Magnetic Donut 8

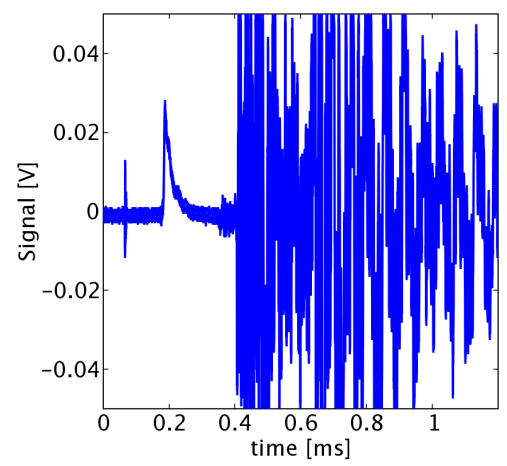

c) Signal from Magnetic Donut 3

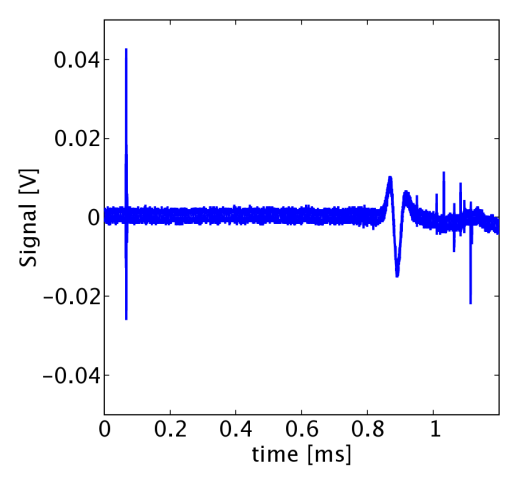

f) Signal from Magnetic Donut 6

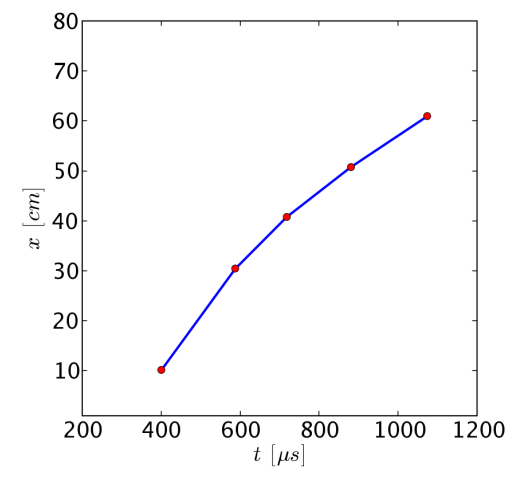

i) Fan plot 
FIG. 27: Krispy Kreme Shot 1397 (1 April 2005): The projectile was a Ta block with square side length 5.0 [mm] and thickness 2.67 [mm], there was a 5\# foam target placed between Magnetic Donut locations 3-6, and 6 Magnetic Donuts were fielded at locations 2-7. X-ray velocity $=1.11[\mathrm{~km} / \mathrm{s}]$.

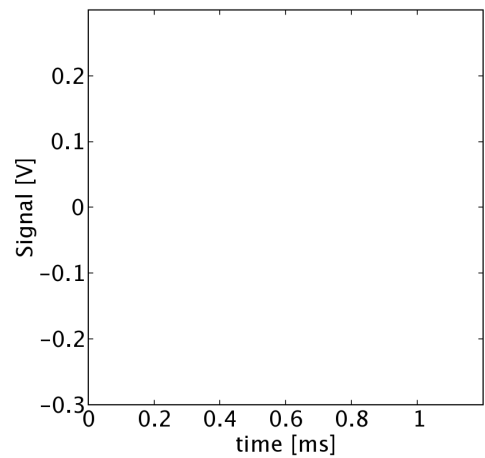

a) Signal from Magnetic Donut 1

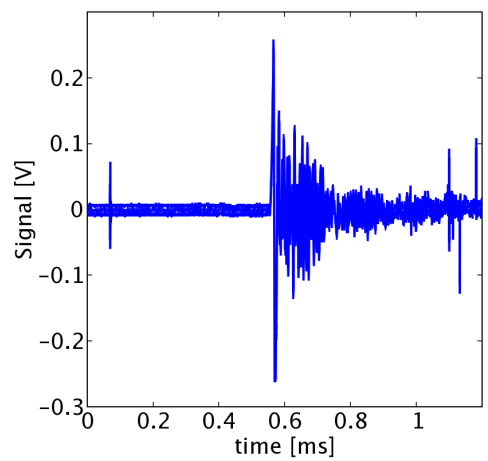

d) Signal from Magnetic Donut 4

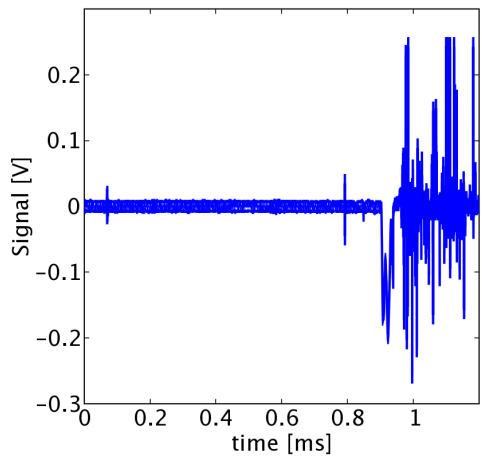

g) Signal from Magnetic Donut 7

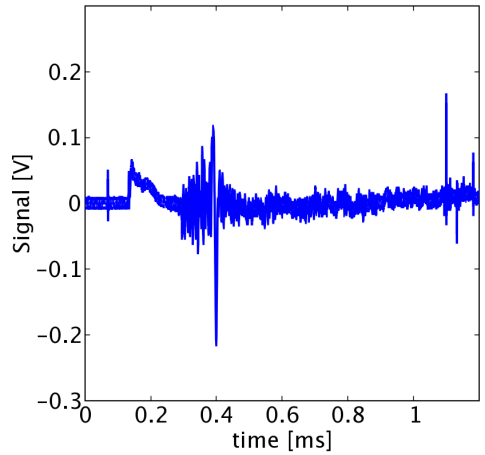

b) Signal from Magnetic Donut 2

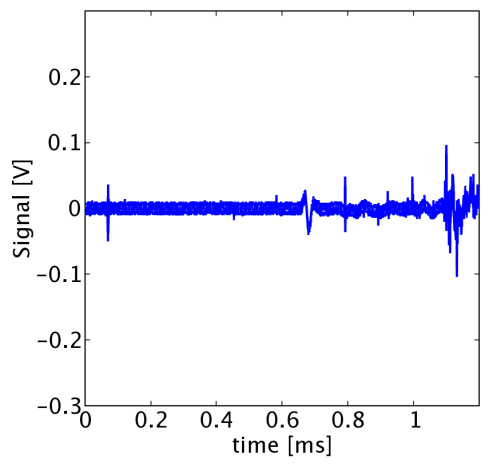

e) Signal from Magnetic Donut 5

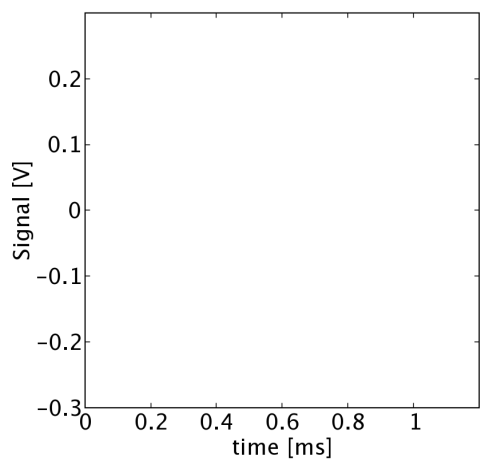

h) Signal from Magnetic Donut 8

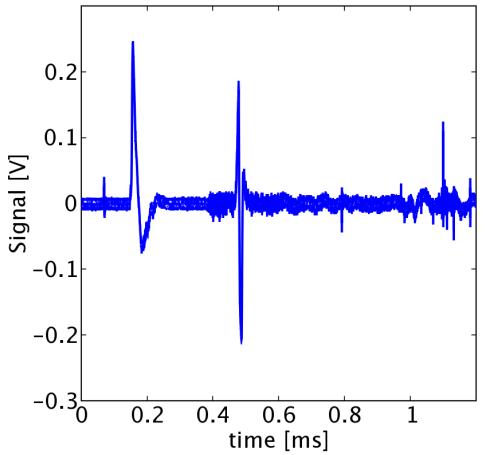

c) Signal from Magnetic Donut 3

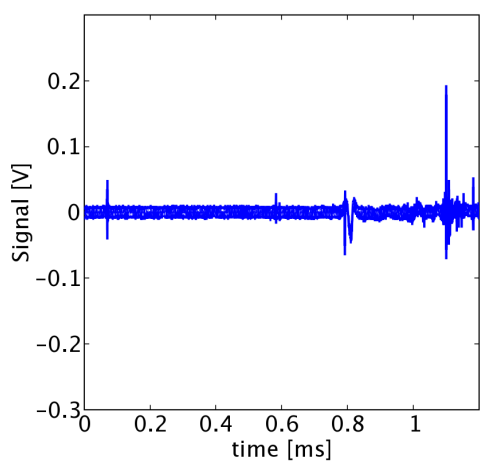

f) Signal from Magnetic Donut 6

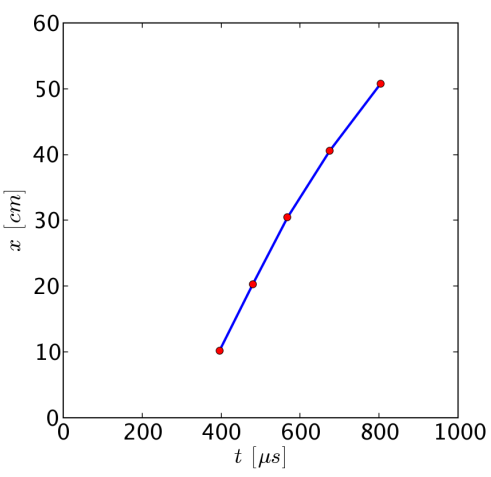

i) Fan plot 
FIG. 28: Krispy Kreme Shot 1398 (4 April 2005): The projectile was a Ta block with square side length 4.0 [mm] and thickness 2.67 [mm], there was a 5\# foam target placed between Magnetic Donut locations 3-6, and 6 Magnetic Donuts were fielded at locations 2-7.

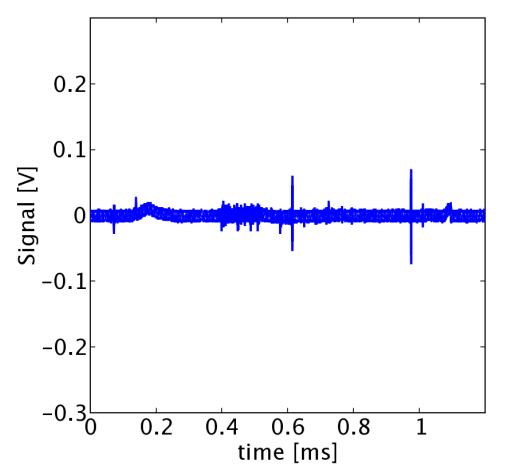

a) Signal from Magnetic Donut 1

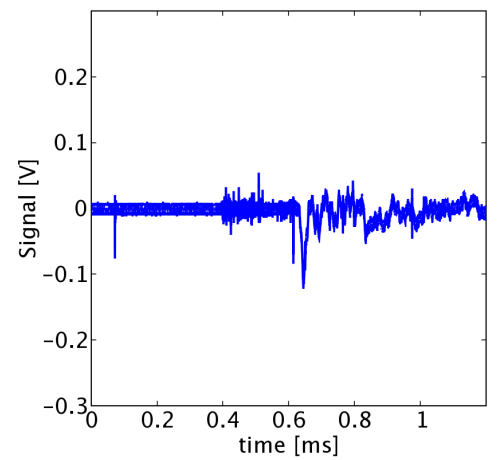

d) Signal from Magnetic Donut 4

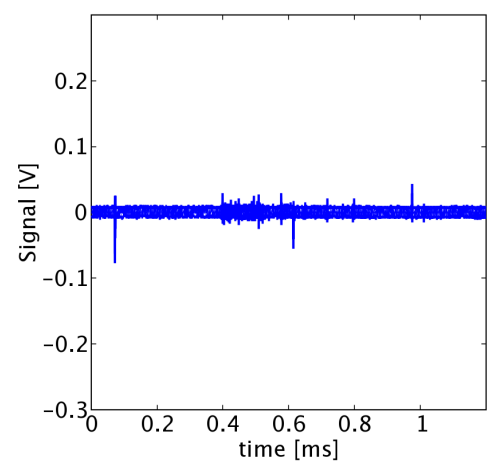

g) Signal from Magnetic Donut 7

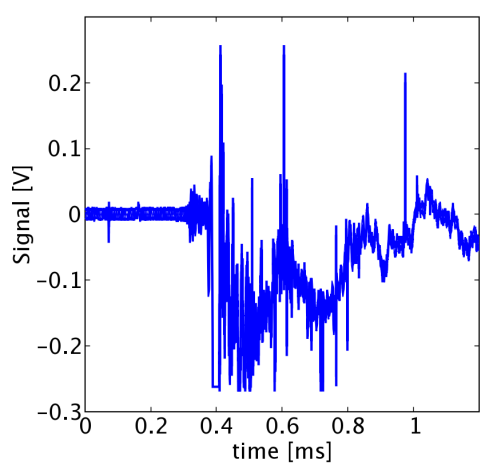

b) Signal from Magnetic Donut 2

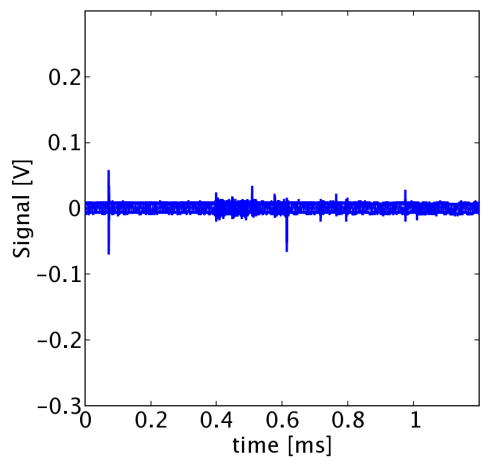

e) Signal from Magnetic Donut 5

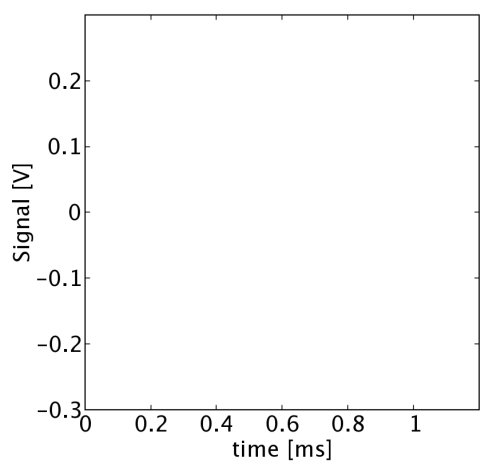

h) Signal from Magnetic Donut 8

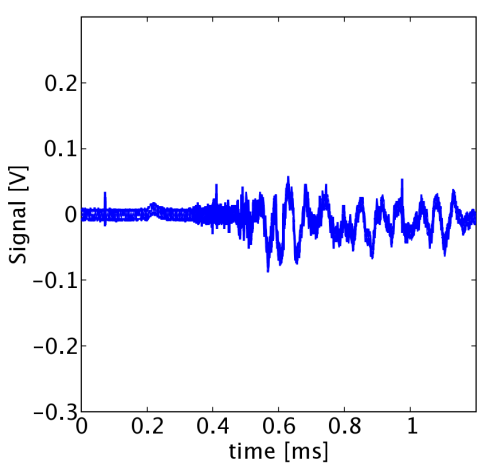

c) Signal from Magnetic Donut 3

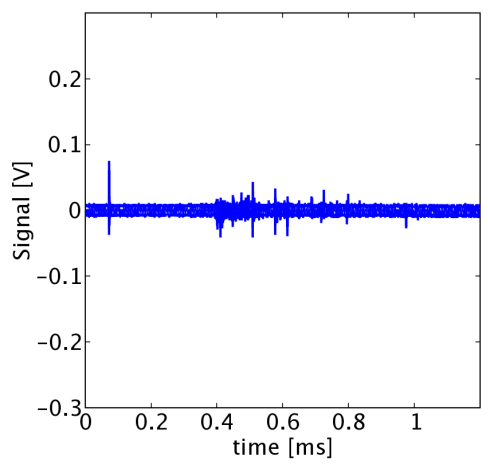

f) Signal from Magnetic Donut 6

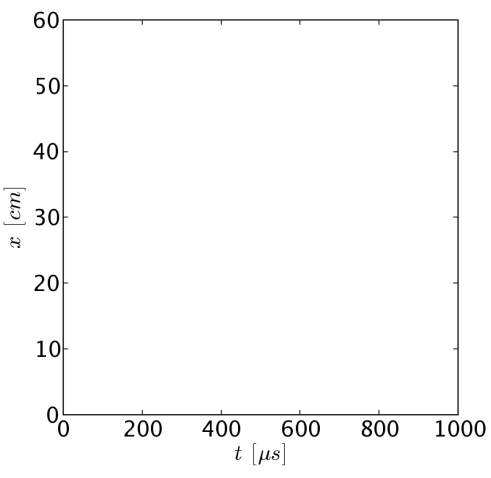

i) Fan plot 
FIG. 29: Krispy Kreme Shot 1399 (5 April 2005): The projectile was a Ta block with square side length 5.0 [mm] and thickness 2.67 [mm], there was a 10\# foam target placed between Magnetic Donut locations 3-6, and 7 Magnetic Donuts were fielded at locations $1-7$. X-ray velocity $=1.13[\mathrm{~km} / \mathrm{s}]$.

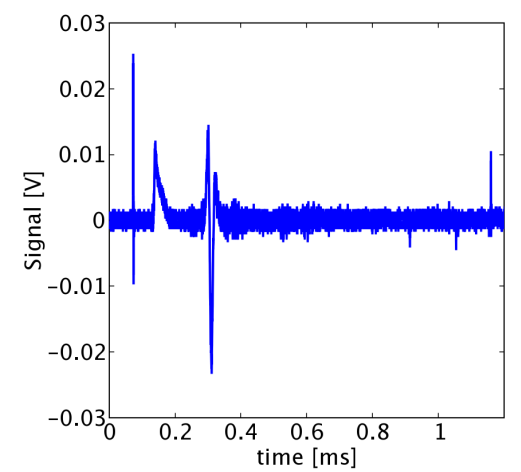

a) Signal from Magnetic Donut 1

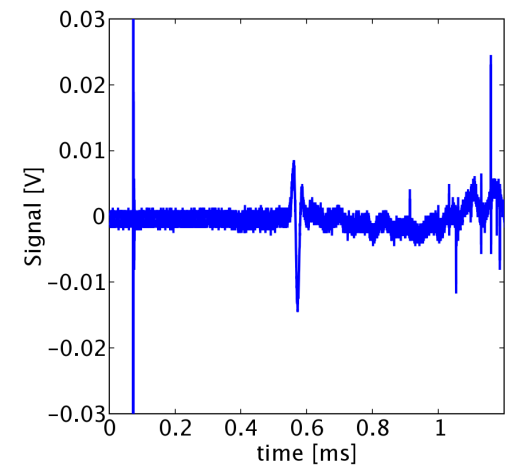

d) Signal from Magnetic Donut 4

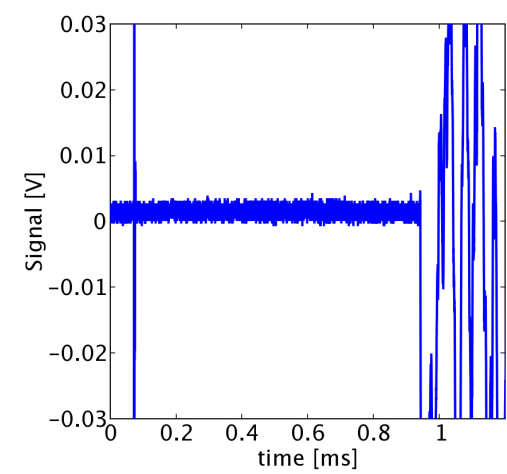

g) Signal from Magnetic Donut 7

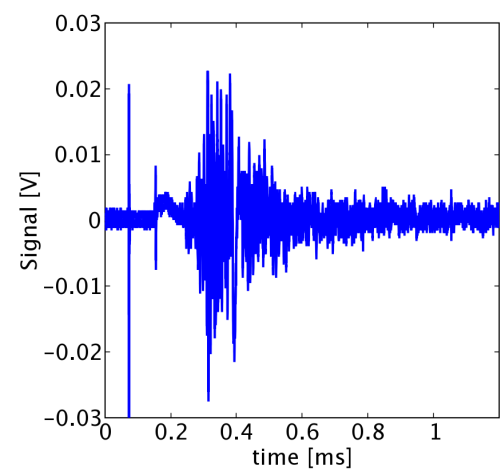

b) Signal from Magnetic Donut 2

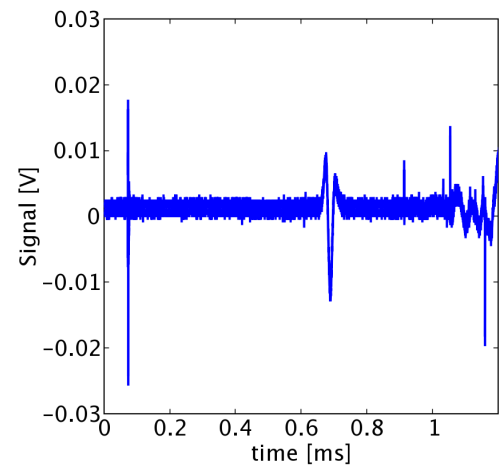

e) Signal from Magnetic Donut 5

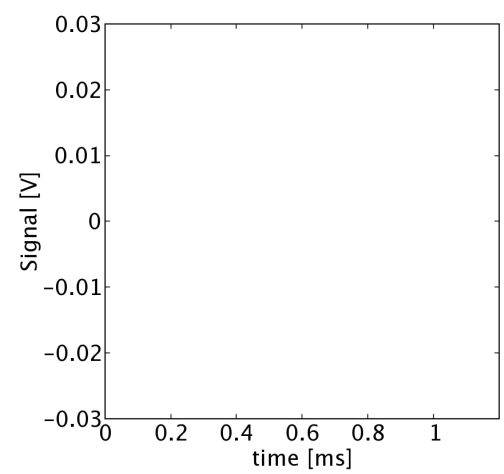

h) Signal from Magnetic Donut 8

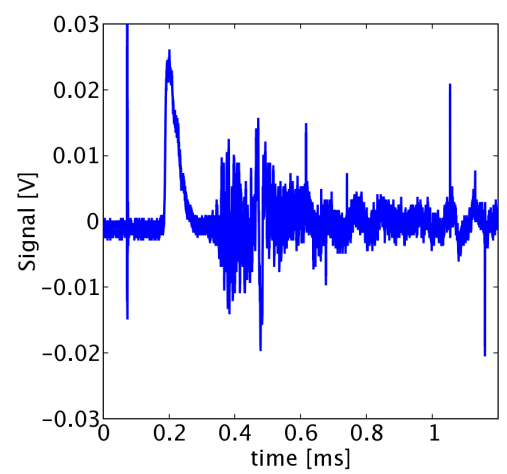

c) Signal from Magnetic Donut 3

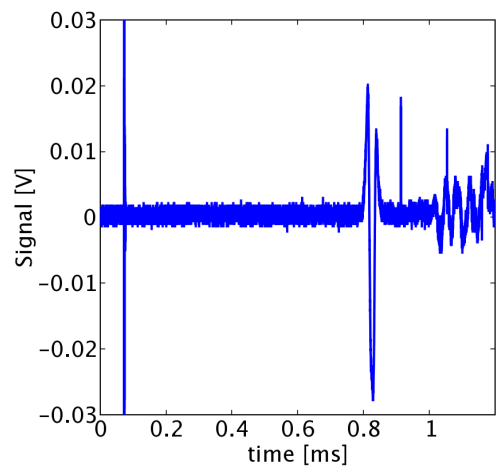

f) Signal from Magnetic Donut 6

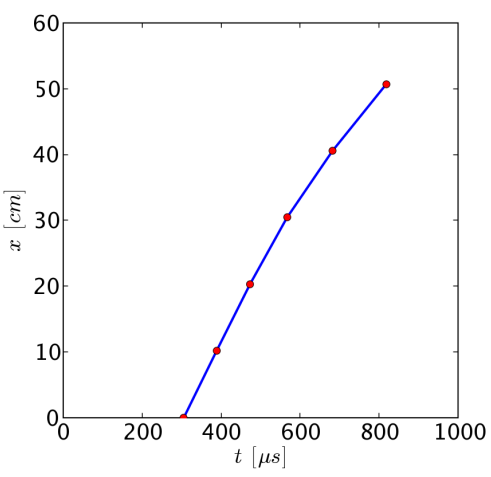

i) Fan plot 
FIG. 30: Krispy Kreme Shot 1400 (6 April 2005): The projectile was a 4.51 [mm] diameter Ta disk with thickness 2.67 [mm], there was a 10\# foam target placed between Magnetic Donut locations 3-6, and 7 Magnetic Donuts were fielded at locations 1-7. $X$-ray velocity $=1.12[\mathrm{~km} / \mathrm{s}]$.

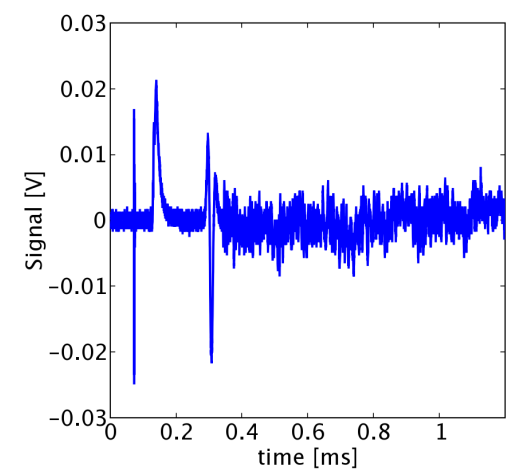

a) Signal from Magnetic Donut 1

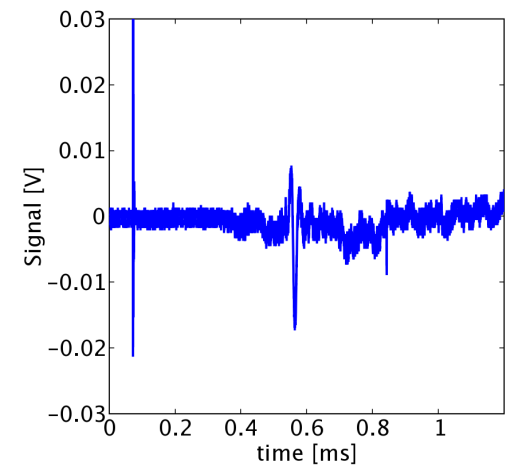

d) Signal from Magnetic Donut 4

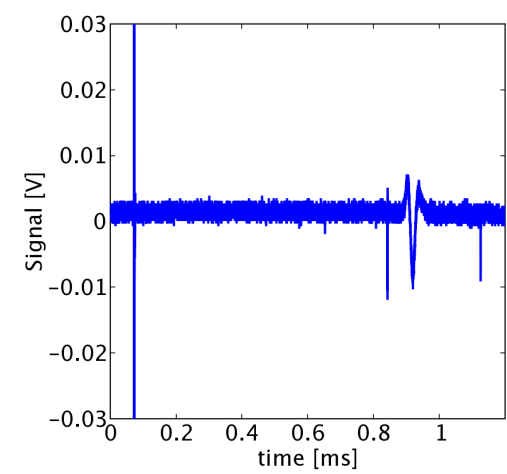

g) Signal from Magnetic Donut 7

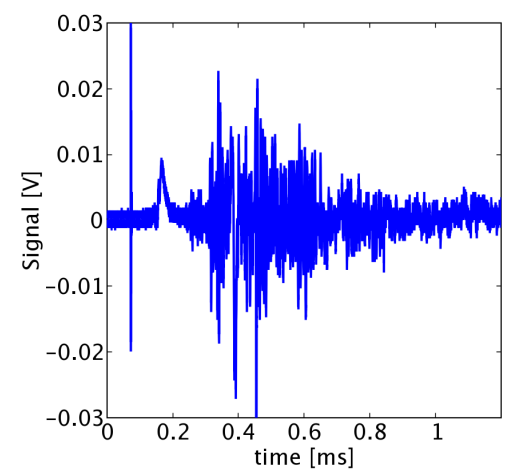

b) Signal from Magnetic Donut 2

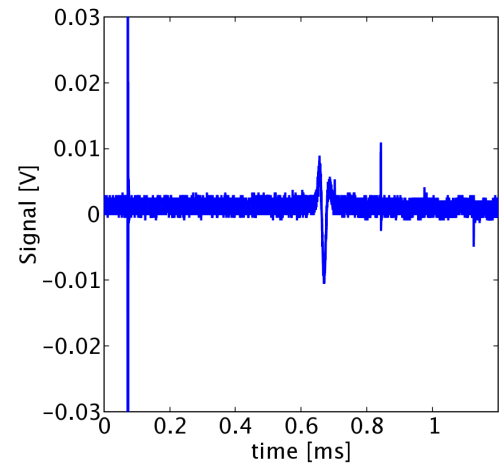

e) Signal from Magnetic Donut 5

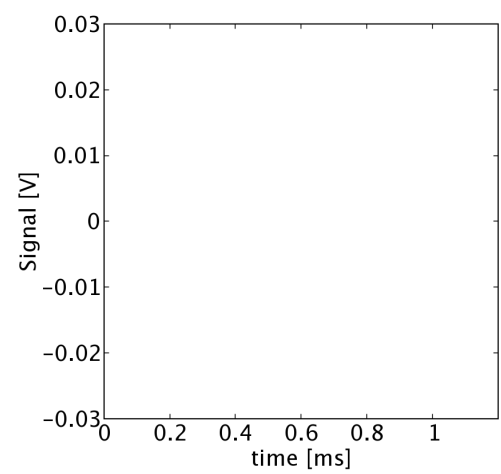

h) Signal from Magnetic Donut 8

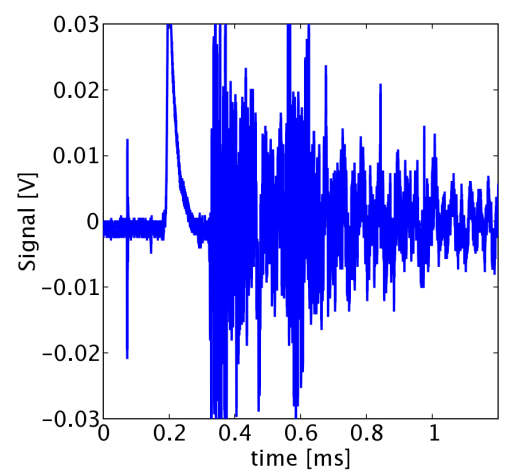

c) Signal from Magnetic Donut 3

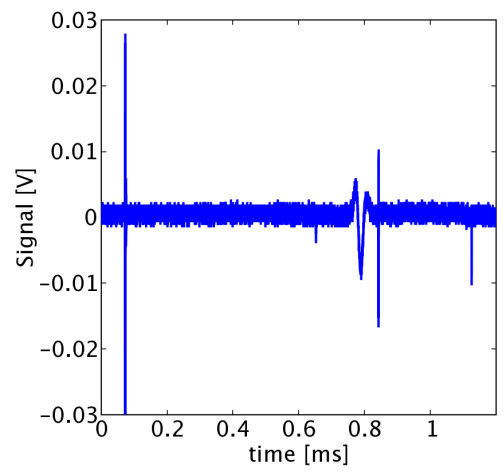

f) Signal from Magnetic Donut 6

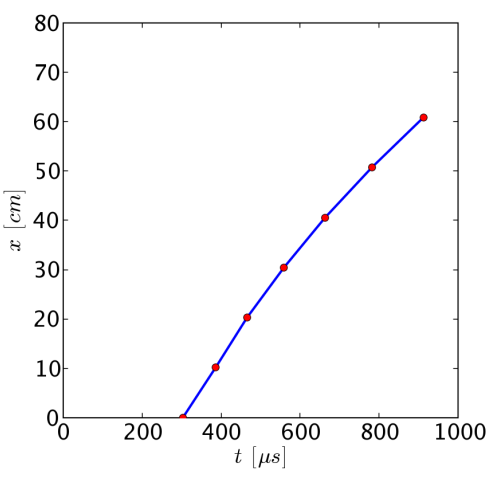

i) Fan plot 
FIG. 31: Krispy Kreme Shot 1401 (6 April 2005): The projectile was a Ta block with square side length 3.0 [mm] and thickness 2.67 [mm], there was a 5\# foam target placed between Magnetic Donut locations 3-6, and 7 Magnetic Donuts were fielded at locations 1-7. X-ray velocity $=1.14[\mathrm{~km} / \mathrm{s}]$.

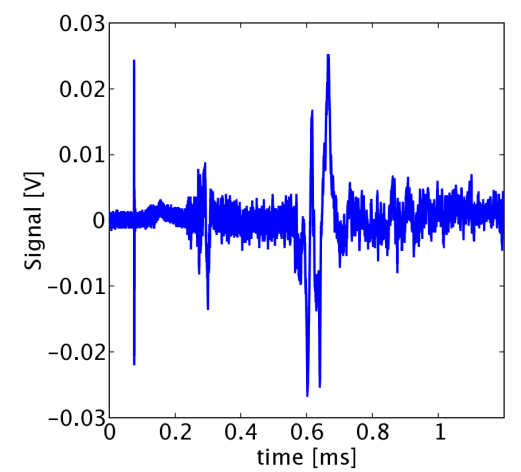

a) Signal from Magnetic Donut 1

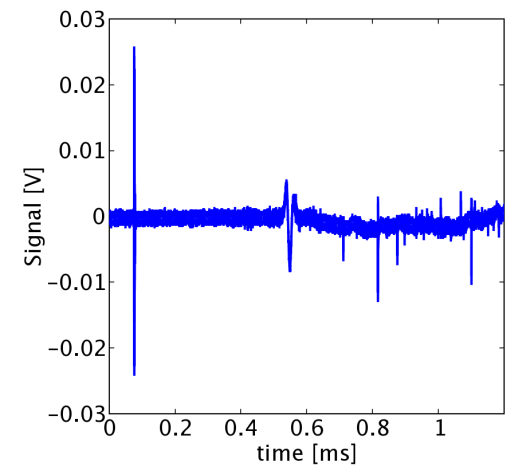

d) Signal from Magnetic Donut 4

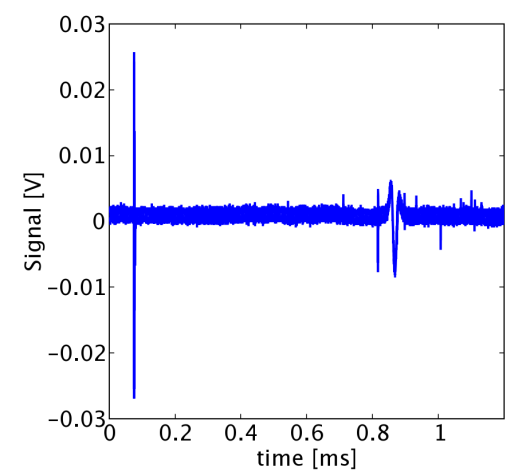

g) Signal from Magnetic Donut 7

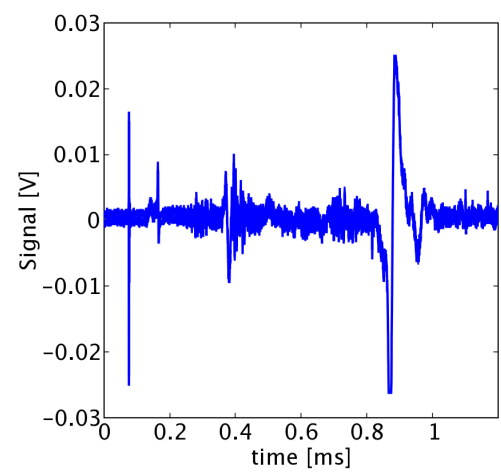

b) Signal from Magnetic Donut 2

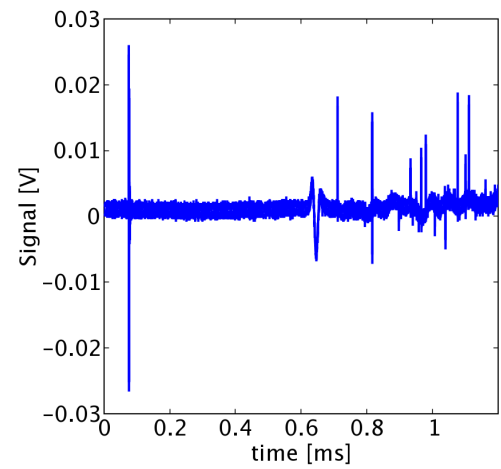

e) Signal from Magnetic Donut 5

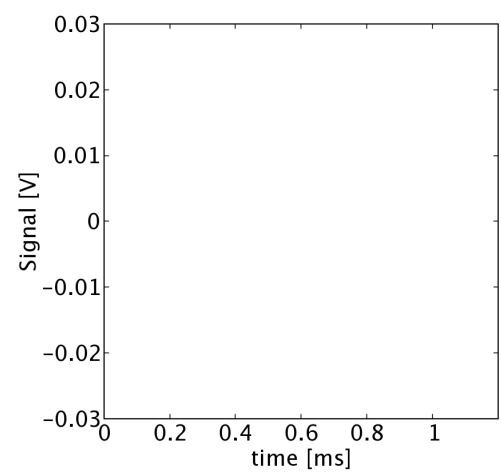

h) Signal from Magnetic Donut 8

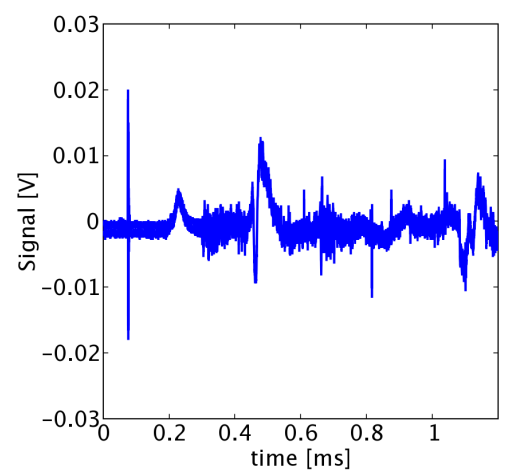

c) Signal from Magnetic Donut 3

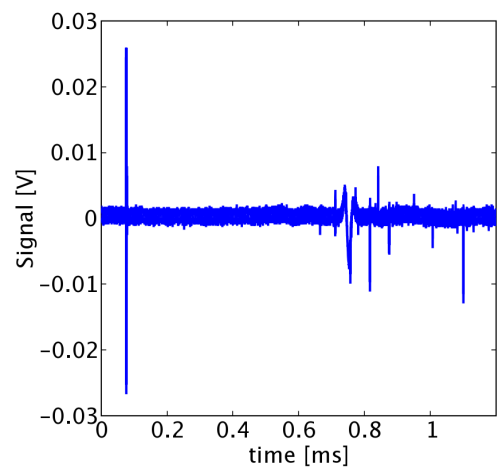

f) Signal from Magnetic Donut 6

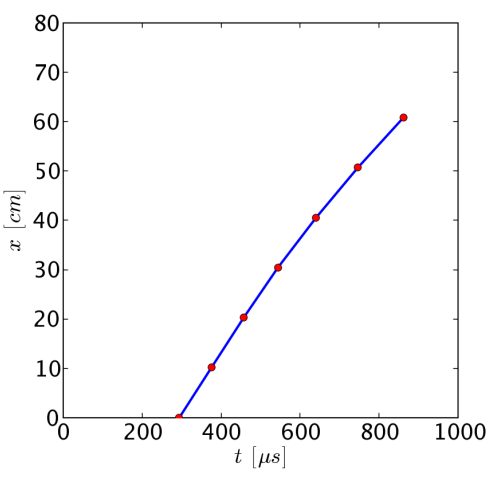

i) Fan plot 
FIG. 32: Krispy Kreme Shot 1402 (7 April 2005): The projectile was a Ta block with square side length 3.0 [mm] and thickness 2.67 [mm], there was a 20\# foam target placed between Magnetic Donut locations 3-6, and 7 Magnetic Donuts were fielded at locations $1-7 . X$-ray velocity $=1.11[\mathrm{~km} / \mathrm{s}]$.

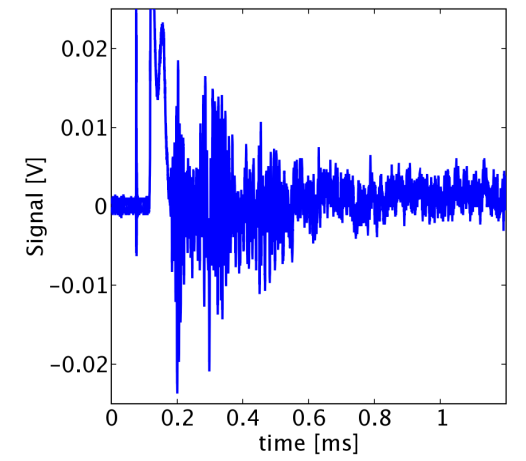

a) Signal from Magnetic Donut 1

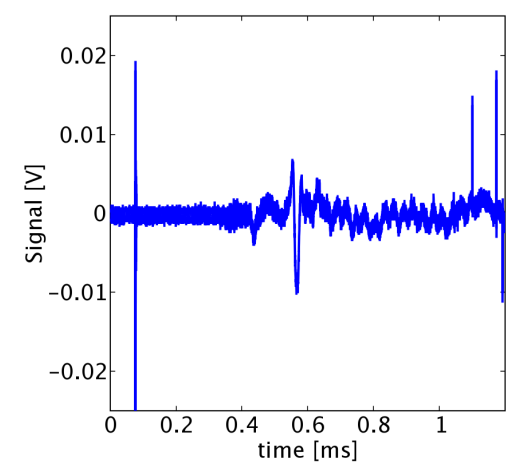

d) Signal from Magnetic Donut 4

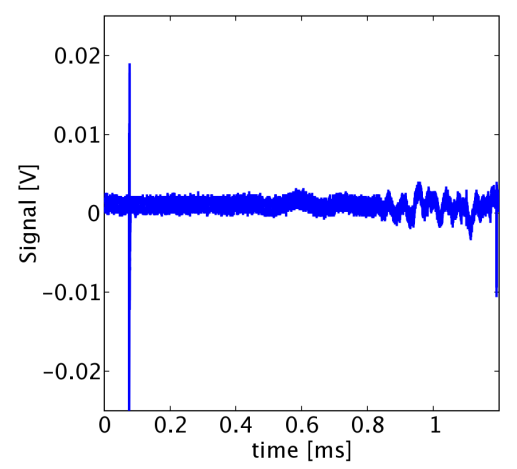

g) Signal from Magnetic Donut 7

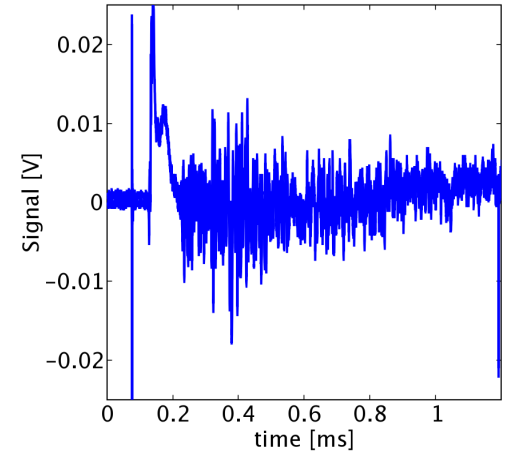

b) Signal from Magnetic Donut 2

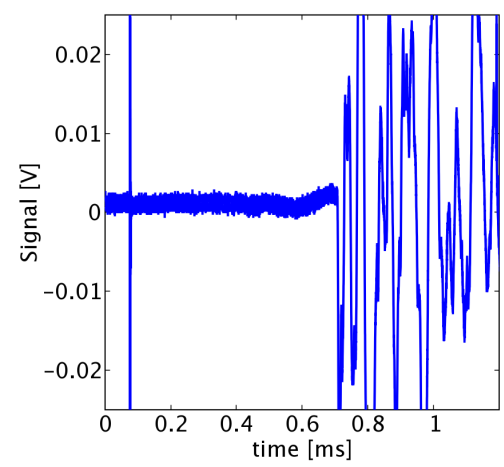

e) Signal from Magnetic Donut 5

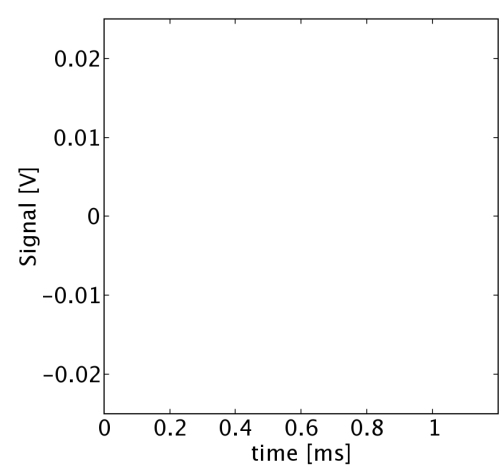

h) Signal from Magnetic Donut 8

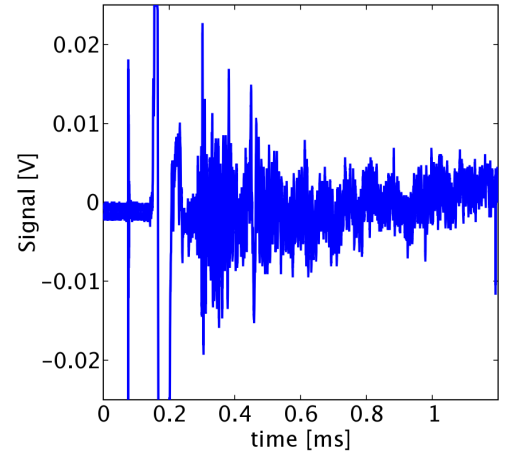

c) Signal from Magnetic Donut 3

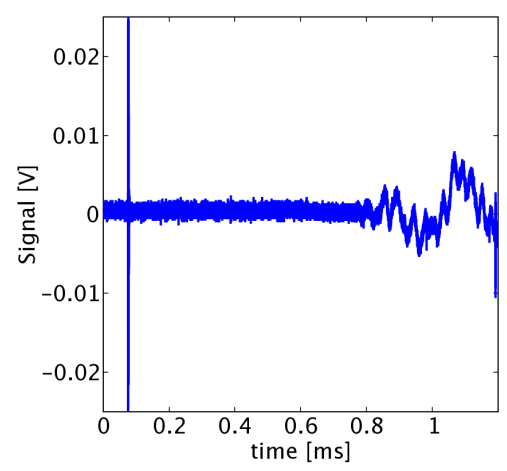

f) Signal from Magnetic Donut 6

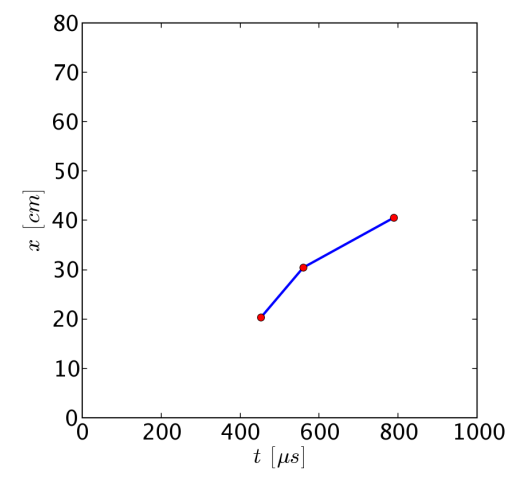

i) Fan plot 
FIG. 33: Krispy Kreme Shot 1403 (8 April 2005): The projectile was a Ta block with square side length 4.0 [mm] and thickness 2.67 [mm], there was a 20\# foam target placed between Magnetic Donut locations 3-6, and 7 Magnetic Donuts were fielded at locations $1-7 . X$-ray velocity $=1.13[\mathrm{~km} / \mathrm{s}]$.

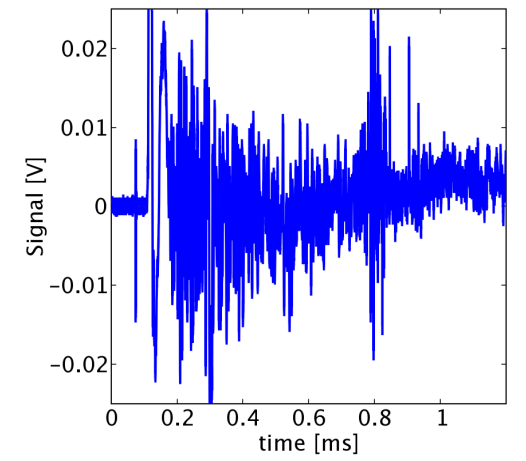

a) Signal from Magnetic Donut 1

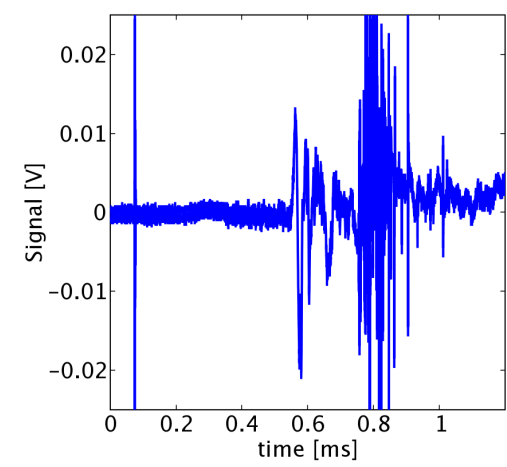

d) Signal from Magnetic Donut 4

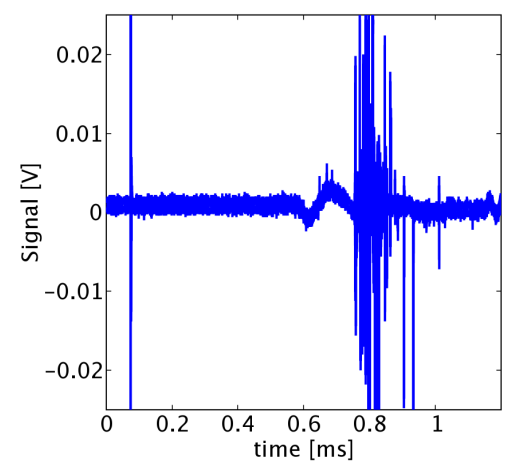

g) Signal from Magnetic Donut 7

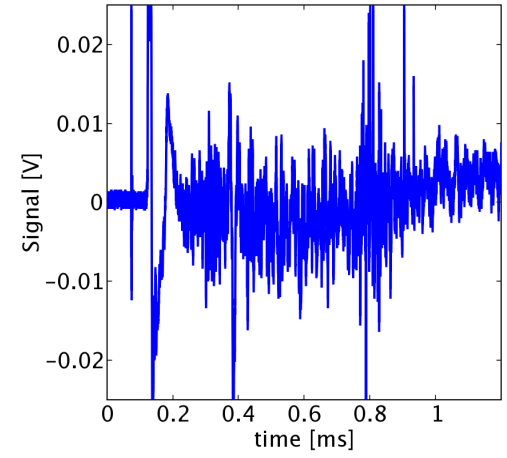

b) Signal from Magnetic Donut 2

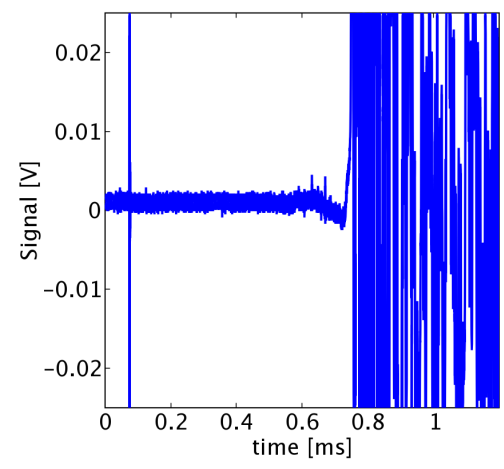

e) Signal from Magnetic Donut 5

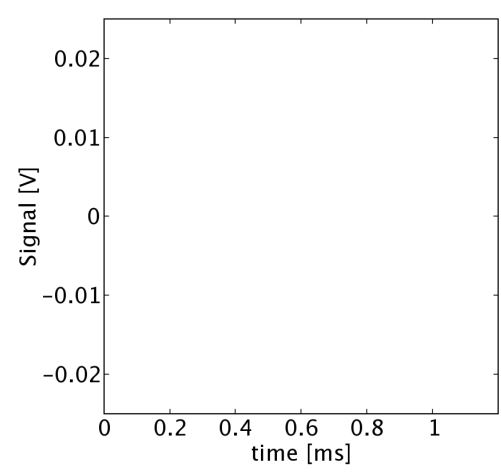

h) Signal from Magnetic Donut 8

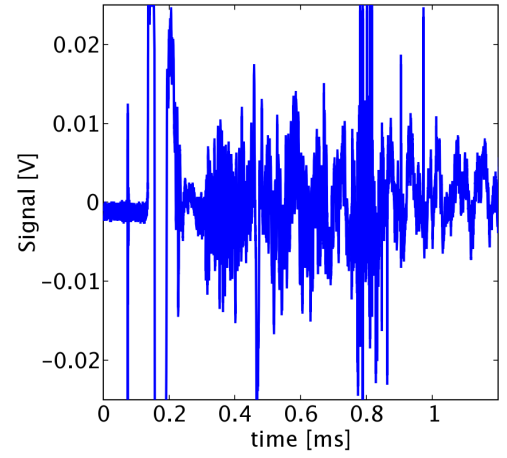

c) Signal from Magnetic Donut 3

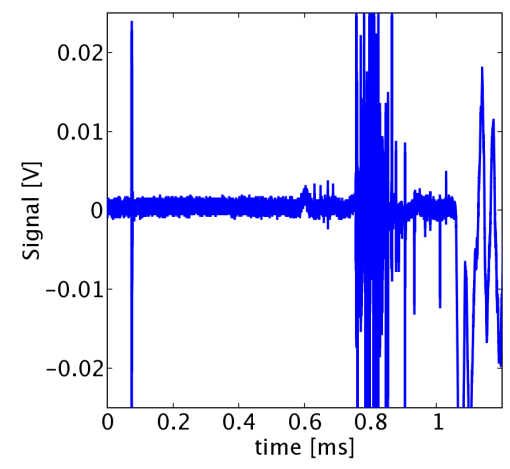

f) Signal from Magnetic Donut 6

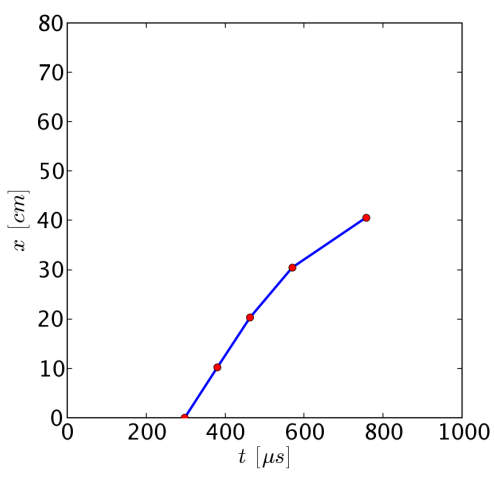

i) Fan plot 
FIG. 34: Krispy Kreme Shot 1404 (8 April 2005): The projectile was a Ta block with square side length 5.0 [mm] and thickness 2.67 [mm], there was a 20\# foam target placed between Magnetic Donut locations 3-6, and 7 Magnetic Donuts were fielded at locations $1-7 . X$-ray velocity $=1.11[\mathrm{~km} / \mathrm{s}]$.

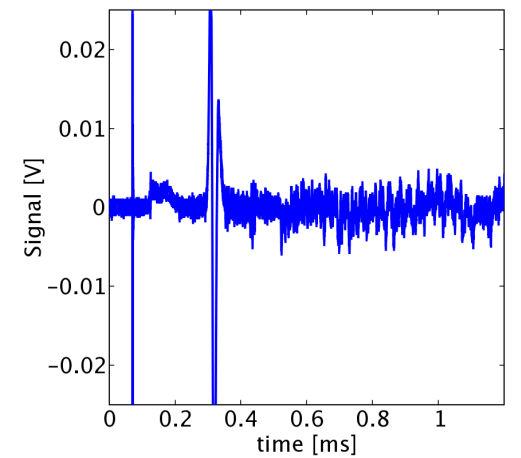

a) Signal from Magnetic Donut 1

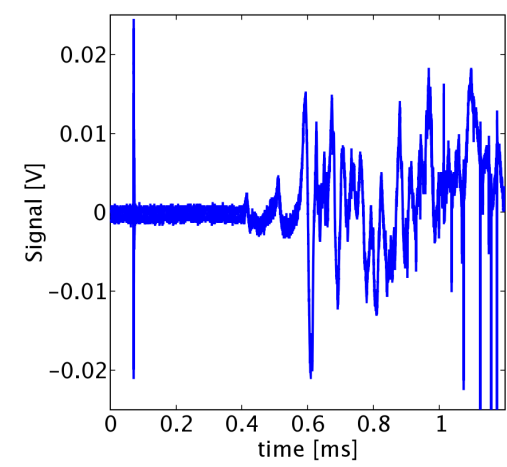

d) Signal from Magnetic Donut 4

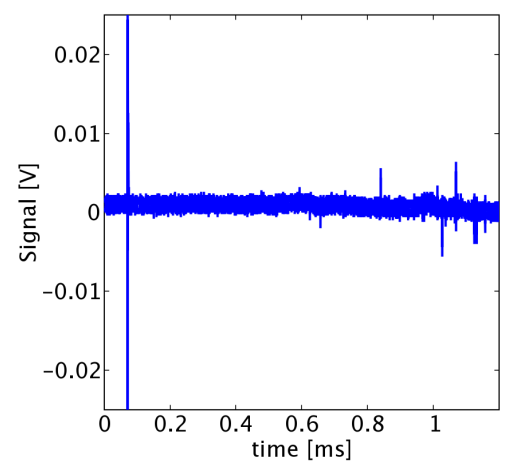

g) Signal from Magnetic Donut 7

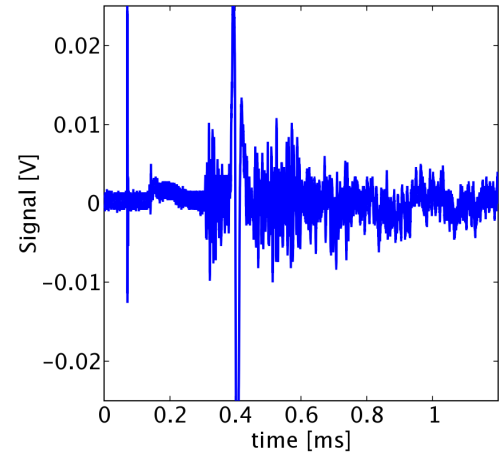

b) Signal from Magnetic Donut 2

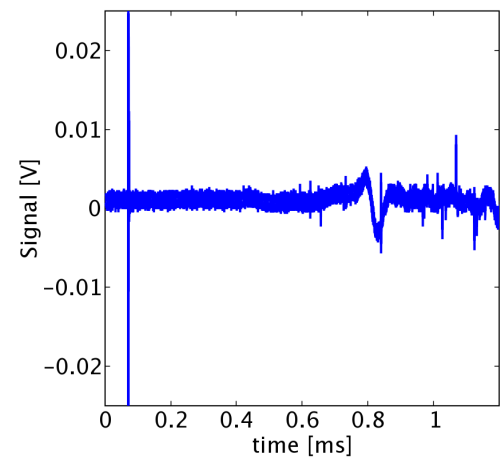

e) Signal from Magnetic Donut 5

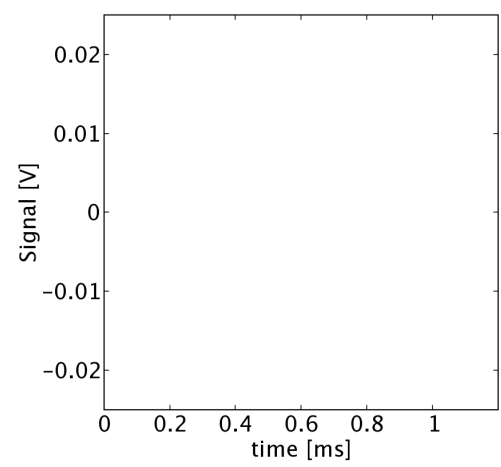

h) Signal from Magnetic Donut 8

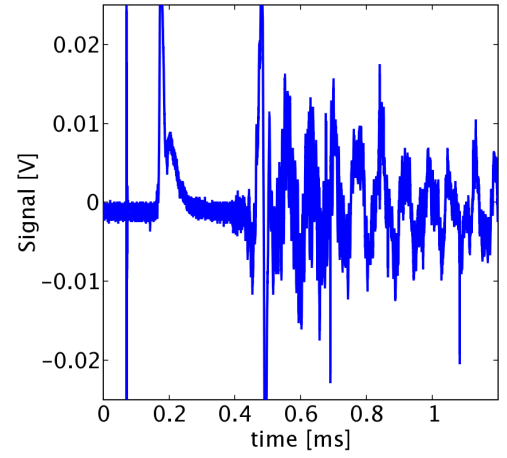

c) Signal from Magnetic Donut 3

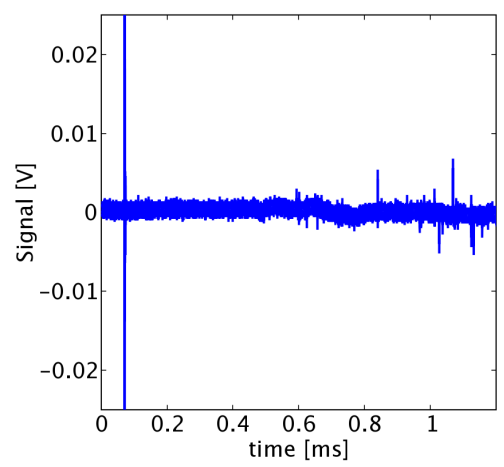

f) Signal from Magnetic Donut 6

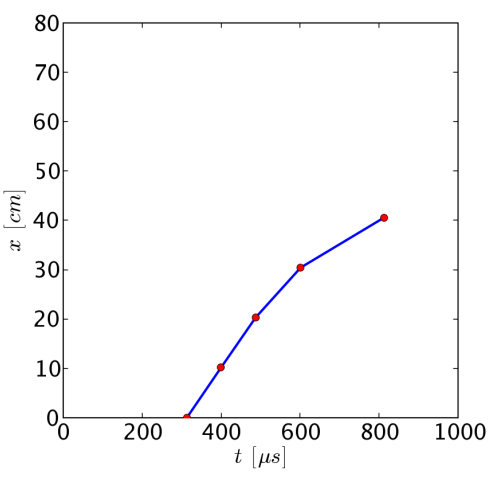

i) Fan plot 
TABLE VIII: Krispy Kreme drag coefficients.

\begin{tabular}{cccccccccc}
\hline \hline donuts A & donuts B & 1396 & 1397 & 1399 & 1400 & 1401 & 1402 & 1403 & 1404 \\
\hline $1-2$ & $3-4$ & 0.0000 & 0.0000 & 1.3591 & 1.3175 & 1.3744 & 0.0000 & 1.4589 & 1.5365 \\
$1-2$ & $4-5$ & 0.0000 & 0.0000 & 1.2024 & 0.8441 & 1.2775 & 0.0000 & 1.5062 & 1.6320 \\
$1-2$ & $5-6$ & 0.0000 & 0.0000 & 1.0863 & 0.7779 & 1.1550 & 0.0000 & 0.0000 & 0.0000 \\
$1-2$ & $6-7$ & 0.0000 & 0.0000 & 0.0000 & 0.8322 & 1.2784 & 0.0000 & 0.0000 & 0.0000 \\
$1-2$ & $7-8$ & 0.0000 & 0.0000 & 0.0000 & 0.0000 & 0.0000 & 0.0000 & 0.0000 & 0.0000 \\
$2-3$ & $3-4$ & 0.0000 & 0.8308 & 1.1201 & 1.7359 & 1.2319 & 0.0000 & 1.3820 & 1.4641 \\
$2-3$ & $4-5$ & 0.0000 & 1.7219 & 1.1228 & 0.9834 & 1.2300 & 0.0000 & 1.4806 & 1.6079 \\
$2-3$ & $5-6$ & 0.0000 & 1.8116 & 1.0386 & 0.8614 & 1.1266 & 0.0000 & 0.0000 & 0.0000 \\
$2-3$ & $6-7$ & 0.0000 & 0.0000 & 0.0000 & 0.9018 & 1.2547 & 0.0000 & 0.0000 & 0.0000 \\
$2-3$ & $7-8$ & 0.0000 & 0.0000 & 0.0000 & 0.0000 & 0.0000 & 0.0000 & 0.0000 & 0.0000 \\
$3-4$ & $4-5$ & 0.0000 & 2.1670 & 1.1241 & 0.6077 & 1.2291 & 2.0834 & 1.5298 & 1.6797 \\
$3-4$ & $5-6$ & 0.0000 & 2.0566 & 1.0183 & 0.6432 & 1.1003 & 0.0000 & 0.0000 & 0.0000 \\
$3-4$ & $6-7$ & 0.0000 & 0.0000 & 0.0000 & 0.7353 & 1.2592 & 0.0000 & 0.0000 & 0.0000 \\
$3-4$ & $7-8$ & 0.0000 & 0.0000 & 0.0000 & 0.0000 & 0.0000 & 0.0000 & 0.0000 & 0.0000 \\
$4-5$ & $5-6$ & 1.3981 & 1.9463 & 0.9126 & 0.6788 & 0.9717 & 0.0000 & 0.0000 & 0.0000 \\
$4-5$ & $6-7$ & 1.5101 & 0.0000 & 0.0000 & 0.8204 & 1.2793 & 0.0000 & 0.0000 & 0.0000 \\
$4-5$ & $7-8$ & 0.0000 & 0.0000 & 0.0000 & 0.0000 & 0.0000 & 0.0000 & 0.0000 & 0.0000 \\
$5-6$ & $6-7$ & 1.7382 & 0.0000 & 0.0000 & 1.1035 & 1.8946 & 0.0000 & 0.0000 & 0.0000 \\
$5-6$ & $7-8$ & 0.0000 & 0.0000 & 0.0000 & 0.0000 & 0.0000 & 0.0000 & 0.0000 & 0.0000 \\
\hline \hline
\end{tabular}

TABLE IX: Krispy Kreme projectile position $x$ and time $t$ coordinates.

\begin{tabular}{|c|c|c|c|c|c|c|c|c|}
\hline donut & $x_{1392[\mathrm{~m}]}$ & $t_{1392}[\mathrm{~s}]$ & $x_{1393}[\mathrm{~m}]$ & $t_{1393}[\mathrm{~s}]$ & $x_{1395}[\mathrm{~m}]$ & $t_{1395}[\mathrm{~s}]$ & $x_{1396}[\mathrm{~m}]$ & $t_{1396}[\mathrm{~s}]$ \\
\hline 1 & 0.0000 & $3.3032 \mathrm{e}-04$ & 0.0000 & $4.0350 \mathrm{e}-05$ & 0.0000 & $0.0000 \mathrm{e}+00$ & 0.0000 & $0.0000 \mathrm{e}+00$ \\
\hline 2 & 0.1016 & $4.2395 \mathrm{e}-04$ & 0.1016 & $1.4595 \mathrm{e}-04$ & 0.1016 & $3.9421 \mathrm{e}-04$ & 0.1016 & $4.0080 \mathrm{e}-04$ \\
\hline 3 & 0.2032 & $0.0000 \mathrm{e}+00$ & 0.2032 & $2.5260 \mathrm{e}-04$ & 0.2032 & $4.8069 \mathrm{e}-04$ & 0.2031 & $0.0000 \mathrm{e}+00$ \\
\hline 4 & 0.3045 & $6.1213 \mathrm{e}-04$ & 0.3045 & $3.5883 \mathrm{e}-04$ & 0.3045 & $5.6682 \mathrm{e}-04$ & 0.3043 & $5.8783 \mathrm{e}-04$ \\
\hline 5 & 0.4060 & $7.0825 \mathrm{e}-04$ & 0.4060 & $4.6700 \mathrm{e}-04$ & 0.4060 & $6.5490 \mathrm{e}-04$ & 0.4077 & $7.1798 \mathrm{e}-04$ \\
\hline 6 & 0.5074 & $8.0120 \mathrm{e}-04$ & 0.5074 & $5.7195 \mathrm{e}-04$ & 0.5073 & $7.3962 \mathrm{e}-04$ & 0.5073 & $8.8000 \mathrm{e}-04$ \\
\hline 7 & 0.6088 & $8.9577 \mathrm{e}-04$ & 0.6088 & $6.7900 \mathrm{e}-04$ & 0.6088 & $8.2637 \mathrm{e}-04$ & 0.6091 & $1.0737 \mathrm{e}-03$ \\
\hline 8 & 0.7103 & $9.8977 \mathrm{e}-04$ & 0.7103 & $7.8545 \mathrm{e}-04$ & 0.7103 & $9.1257 \mathrm{e}-04$ & 0.7104 & $0.0000 \mathrm{e}+00$ \\
\hline donut & $x_{1397}[\mathrm{~m}]$ & $t_{1397}[\mathrm{~s}]$ & $x_{1399}[\mathrm{~m}]$ & $t_{1399}[\mathrm{~s}]$ & $x_{1400}[\mathrm{~m}]$ & $t_{1400}[\mathrm{~s}]$ & $x_{1401}[\mathrm{~m}]$ & $t_{1401}[\mathrm{~s}]$ \\
\hline 1 & 0.0000 & $0.0000 \mathrm{e}+00$ & 0.0000 & $3.0530 \mathrm{e}-04$ & 0.0000 & $3.0280 \mathrm{e}-04$ & 0.0000 & $2.9387 \mathrm{e}-04$ \\
\hline 2 & 0.1016 & $3.9558 \mathrm{e}-04$ & 0.1016 & $3.8840 \mathrm{e}-04$ & 0.1016 & $3.8585 \mathrm{e}-04$ & 0.1016 & $3.7540 \mathrm{e}-04$ \\
\hline 3 & 0.2032 & $4.8031 \mathrm{e}-04$ & 0.2032 & $4.7333 \mathrm{e}-04$ & 0.2032 & $4.6573 \mathrm{e}-04$ & 0.2032 & $4.5740 \mathrm{e}-04$ \\
\hline 4 & 0.3045 & $5.6815 \mathrm{e}-04$ & 0.3043 & $5.6690 \mathrm{e}-04$ & 0.3043 & $5.5890 \mathrm{e}-04$ & 0.3043 & $5.4375 \mathrm{e}-04$ \\
\hline 5 & 0.4060 & $6.7545 \mathrm{e}-04$ & 0.4057 & $6.8215 \mathrm{e}-04$ & 0.4057 & $6.6330 \mathrm{e}-04$ & 0.4057 & $6.4063 \mathrm{e}-04$ \\
\hline 6 & 0.5074 & $8.0345 \mathrm{e}-04$ & 0.5071 & $8.1834 \mathrm{e}-04$ & 0.5071 & $7.8150 \mathrm{e}-04$ & 0.5071 & $7.4650 \mathrm{e}-04$ \\
\hline 7 & 0.6088 & $0.0000 \mathrm{e}+00$ & 0.6088 & $0.0000 \mathrm{e}+00$ & 0.6088 & $9.1270 \mathrm{e}-04$ & 0.6088 & $8.6235 \mathrm{e}-04$ \\
\hline 8 & 0.7103 & $0.0000 \mathrm{e}+00$ & 0.7103 & $0.0000 \mathrm{e}+00$ & 0.7103 & $0.0000 \mathrm{e}+00$ & 0.7103 & $0.0000 \mathrm{e}+00$ \\
\hline donut & $x_{1402}[\mathrm{~m}]$ & $t_{1402}[\mathrm{~s}]$ & $x_{1403}[\mathrm{~m}]$ & $t_{1403}[\mathrm{~s}]$ & $x_{1404}[\mathrm{~m}]$ & $t_{1404}[\mathrm{~s}]$ & & \\
\hline 1 & 0.0000 & $0.0000 \mathrm{e}+00$ & 0.0000 & $2.9759 \mathrm{e}-04$ & 0.0000 & $3.1302 \mathrm{e}-04$ & & \\
\hline 2 & 0.1017 & $0.0000 \mathrm{e}+00$ & 0.1017 & $3.7978 \mathrm{e}-04$ & 0.1017 & $3.9969 \mathrm{e}-04$ & & \\
\hline 3 & 0.2032 & $4.5262 \mathrm{e}-04$ & 0.2032 & $4.6307 \mathrm{e}-04$ & 0.2032 & $4.8742 \mathrm{e}-04$ & & \\
\hline 4 & 0.3044 & $5.5975 \mathrm{e}-04$ & 0.3043 & $5.6975 \mathrm{e}-04$ & 0.3043 & $6.0148 \mathrm{e}-04$ & & \\
\hline 5 & 0.4057 & $7.8984 \mathrm{e}-04$ & 0.4057 & $7.5691 \mathrm{e}-04$ & 0.4057 & $8.1290 \mathrm{e}-04$ & & \\
\hline 6 & 0.5071 & $0.0000 \mathrm{e}+00$ & 0.5071 & $0.0000 \mathrm{e}+00$ & 0.5070 & $0.0000 \mathrm{e}+00$ & & \\
\hline 7 & 0.6088 & $0.0000 \mathrm{e}+00$ & 0.6088 & $0.0000 \mathrm{e}+00$ & 0.6086 & $0.0000 \mathrm{e}+00$ & & \\
\hline 8 & 0.7103 & $0.0000 \mathrm{e}+00$ & 0.7103 & $0.0000 \mathrm{e}+00$ & 0.7101 & $0.0000 \mathrm{e}+00$ & & \\
\hline
\end{tabular}


TABLE X: Krispy Kreme projectile velocities.

\begin{tabular}{|c|c|c|c|c|c|c|c|}
\hline donut $\mathrm{A}$ & donut B & $v_{1392}[\mathrm{~m} / \mathrm{s}]$ & $v_{1393}[\mathrm{~m} / \mathrm{s}]$ & $v_{1395}[\mathrm{~m} / \mathrm{s}]$ & $v_{1396}[\mathrm{~m} / \mathrm{s}]$ & $v_{1397}[\mathrm{~m} / \mathrm{s}]$ & $v_{1399}[\mathrm{~m} / \mathrm{s}]$ \\
\hline 1 & 2 & 1085.2825 & 962.2632 & 0.0000 & 0.0000 & 0.0000 & 1222.9768 \\
\hline 1 & 3 & 0.0000 & 957.3381 & 0.0000 & 0.0000 & 0.0000 & 1209.6402 \\
\hline 1 & 4 & 1080.5202 & 956.1347 & 0.0000 & 0.0000 & 0.0000 & 1163.3788 \\
\hline 1 & 5 & 1074.3524 & 951.6700 & 0.0000 & 0.0000 & 0.0000 & 1076.6084 \\
\hline 1 & 6 & 1077.5251 & 954.4488 & 0.0000 & 0.0000 & 0.0000 & 988.4023 \\
\hline 1 & 7 & 1076.7328 & 953.3156 & 0.0000 & 0.0000 & 0.0000 & 0.0000 \\
\hline 1 & 8 & 1077.0848 & 953.2680 & 0.0000 & 0.0000 & 0.0000 & 0.0000 \\
\hline 2 & 3 & 0.0000 & 952.4613 & 1174.6983 & 0.0000 & 1198.5954 & 1196.5900 \\
\hline 2 & 4 & 1078.1507 & 953.0946 & 1175.6580 & 1083.8512 & 1175.8436 & 1135.6329 \\
\hline 2 & 5 & 1070.7527 & 948.1856 & 1167.7968 & 965.0084 & 1087.7906 & 1035.2014 \\
\hline 2 & 6 & 1075.5997 & 952.5117 & 1174.6919 & 846.5730 & 994.8758 & 943.0627 \\
\hline 2 & 7 & 1075.0362 & 951.5430 & 1173.6387 & 754.2286 & 0.0000 & 0.0000 \\
\hline 2 & 8 & 1075.7283 & 951.7826 & 1174.3128 & 0.0000 & 0.0000 & 0.0000 \\
\hline 3 & 4 & 0.0000 & 953.7303 & 1176.6215 & 0.0000 & 1153.8949 & 1080.3108 \\
\hline 3 & 5 & 0.0000 & 946.0588 & 1164.3712 & 0.0000 & 1039.6770 & 969.5681 \\
\hline 3 & 6 & 0.0000 & 952.5286 & 1174.6897 & 0.0000 & 941.4568 & 880.6576 \\
\hline 3 & 7 & 0.0000 & 951.3133 & 1173.3737 & 0.0000 & 0.0000 & 0.0000 \\
\hline 3 & 8 & 0.0000 & 951.6468 & 1174.2357 & 0.0000 & 0.0000 & 0.0000 \\
\hline 4 & 5 & 1056.2685 & 938.5255 & 1152.3909 & 794.2318 & 946.1852 & 879.6528 \\
\hline 4 & 6 & 1073.0606 & 951.9296 & 1173.7268 & 694.6881 & 862.1593 & 806.3554 \\
\hline 4 & 7 & 1072.9698 & 950.5114 & 1172.2959 & 627.3489 & 0.0000 & 0.0000 \\
\hline 4 & 8 & 1074.5211 & 951.1280 & 1173.6413 & 0.0000 & 0.0000 & 0.0000 \\
\hline 5 & 6 & 1090.4249 & 965.7456 & 1195.9078 & 614.7275 & 791.7189 & 744.3279 \\
\hline 5 & 7 & 1081.5305 & 956.6274 & 1182.5201 & 566.2911 & 0.0000 & 0.0000 \\
\hline 5 & 8 & 1080.7531 & 955.4090 & 1180.9053 & 0.0000 & 0.0000 & 0.0000 \\
\hline 6 & 7 & 1072.7882 & 947.6880 & 1169.4455 & 525.7754 & 0.0000 & 0.0000 \\
\hline 6 & 8 & 1075.9855 & 950.3279 & 1173.5558 & 0.0000 & 0.0000 & 0.0000 \\
\hline 7 & 8 & 1079.2021 & 952.9826 & 1177.6926 & 0.0000 & 0.0000 & 0.0000 \\
\hline
\end{tabular}

\begin{tabular}{|c|c|c|c|c|c|c|}
\hline donut $\mathrm{A}$ & donut B & $v_{1400}[\mathrm{~m} / \mathrm{s}]$ & $v_{1401}[\mathrm{~m} / \mathrm{s}]$ & $v_{1402}[\mathrm{~m} / \mathrm{s}]$ & $v_{1403}[\mathrm{~m} / \mathrm{s}]$ & $v_{1404}[\mathrm{~m} / \mathrm{s}]$ \\
\hline 1 & 2 & 1223.8403 & 1246.7356 & 0.0000 & 1236.8496 & 1173.0464 \\
\hline 1 & 3 & 1247.1987 & 1242.6840 & 0.0000 & 1228.1732 & 1165.2929 \\
\hline 1 & 4 & 1188.2467 & 1217.8883 & 0.0000 & 1118.2184 & 1054.9406 \\
\hline 1 & 5 & 1125.3530 & 1170.0063 & 0.0000 & 883.3043 & 811.5917 \\
\hline 1 & 6 & 1059.2427 & 1120.2871 & 0.0000 & 0.0000 & 0.0000 \\
\hline 1 & 7 & 998.1470 & 1070.8996 & 0.0000 & 0.0000 & 0.0000 \\
\hline 1 & 8 & 0.0000 & 0.0000 & 0.0000 & 0.0000 & 0.0000 \\
\hline 2 & 3 & 1271.4855 & 1238.6560 & 0.0000 & 1219.6110 & 1157.6326 \\
\hline 2 & 4 & 1171.1646 & 1203.9187 & 0.0000 & 1066.8919 & 1004.2088 \\
\hline 2 & 5 & 1095.8725 & 1146.4213 & 0.0000 & 806.2513 & 735.7713 \\
\hline 2 & 6 & 1024.6924 & 1092.5083 & 0.0000 & 0.0000 & 0.0000 \\
\hline 2 & 7 & 962.5698 & 1041.4613 & 0.0000 & 0.0000 & 0.0000 \\
\hline 2 & 8 & 0.0000 & 0.0000 & 0.0000 & 0.0000 & 0.0000 \\
\hline 3 & 4 & 1085.1635 & 1170.9314 & 943.7134 & 947.6626 & 886.1957 \\
\hline 3 & 5 & 1024.8761 & 1105.1428 & 600.4307 & 689.0843 & 622.0599 \\
\hline 3 & 6 & 962.2663 & 1051.0551 & 0.0000 & 0.0000 & 0.0000 \\
\hline 3 & 7 & 907.3661 & 1001.5305 & 0.0000 & 0.0000 & 0.0000 \\
\hline 3 & 8 & 0.0000 & 0.0000 & 0.0000 & 0.0000 & 0.0000 \\
\hline 4 & 5 & 971.0710 & 1046.5018 & 440.6014 & 541.6880 & 479.5664 \\
\hline 4 & 6 & 910.8246 & 1000.0004 & 0.0000 & 0.0000 & 0.0000 \\
\hline 4 & 7 & 860.5424 & 955.6178 & 0.0000 & 0.0000 & 0.0000 \\
\hline 4 & 8 & 0.0000 & 0.0000 & 0.0000 & 0.0000 & 0.0000 \\
\hline 5 & 6 & 857.6122 & 957.4518 & 0.0000 & 0.0000 & 0.0000 \\
\hline 5 & 7 & 814.2745 & 915.9092 & 0.0000 & 0.0000 & 0.0000 \\
\hline 5 & 8 & 0.0000 & 0.0000 & 0.0000 & 0.0000 & 0.0000 \\
\hline 6 & 7 & 775.2307 & 877.9437 & 0.0000 & 0.0000 & 0.0000 \\
\hline 6 & 8 & 0.0000 & 0.0000 & 0.0000 & 0.0000 & 0.0000 \\
\hline 7 & 8 & 0.0000 & 0.0000 & 0.0000 & 0.0000 & 0.0000 \\
\hline
\end{tabular}


APPENDIX C: FIRING RANGE EXPERIMENTS ON 13 APRIL 2005

FIG. 35: Range Day 3, Shot 2
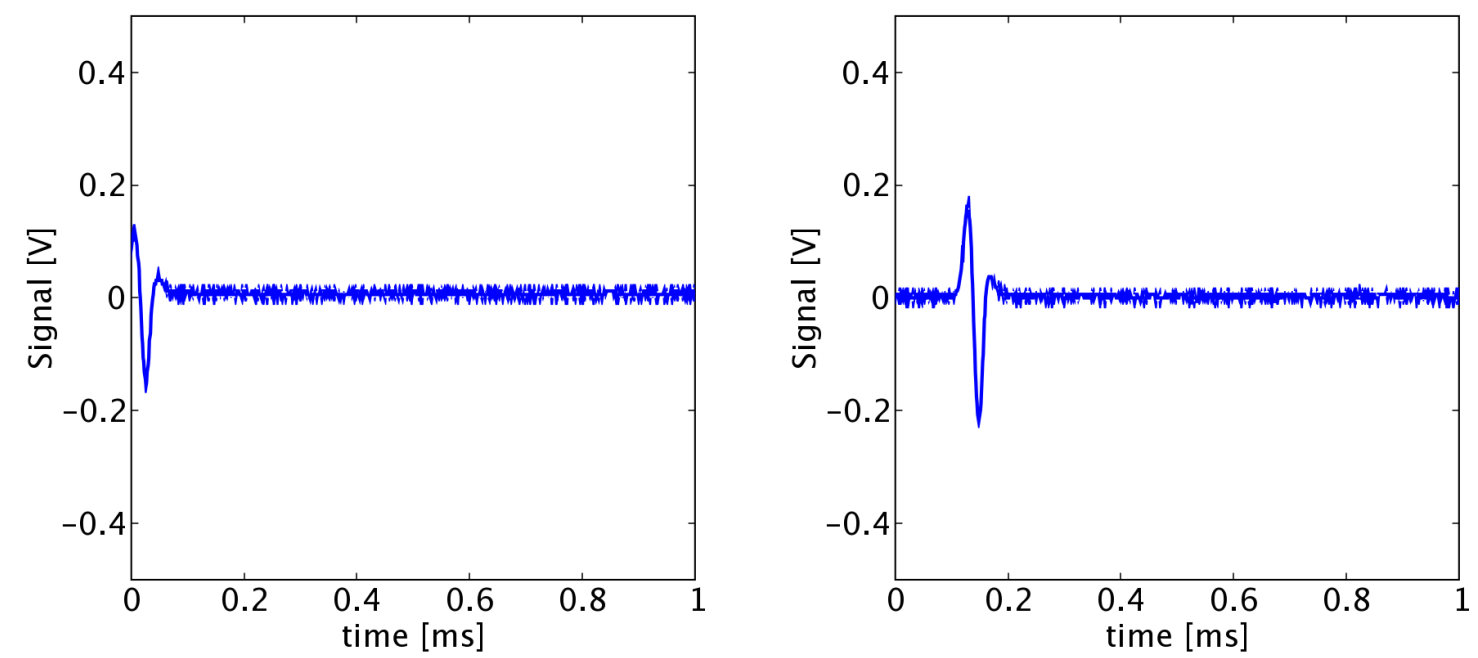

a) Signal from Magnetic Donut 1

b) Signal from Magnetic Donut 2
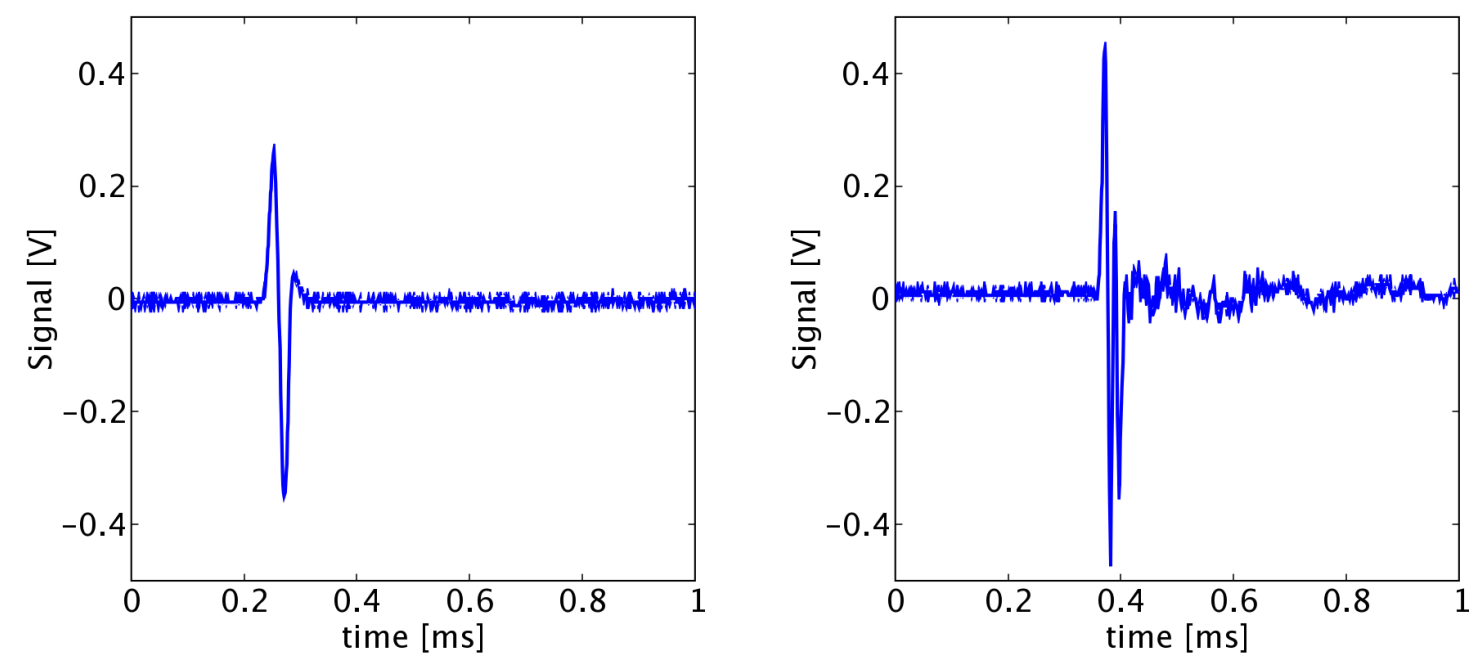

c) Signal from Magnetic Donut 3

d) Signal from Magnetic Donut 4 
FIG. 36: Range Day, 3 Shot 3

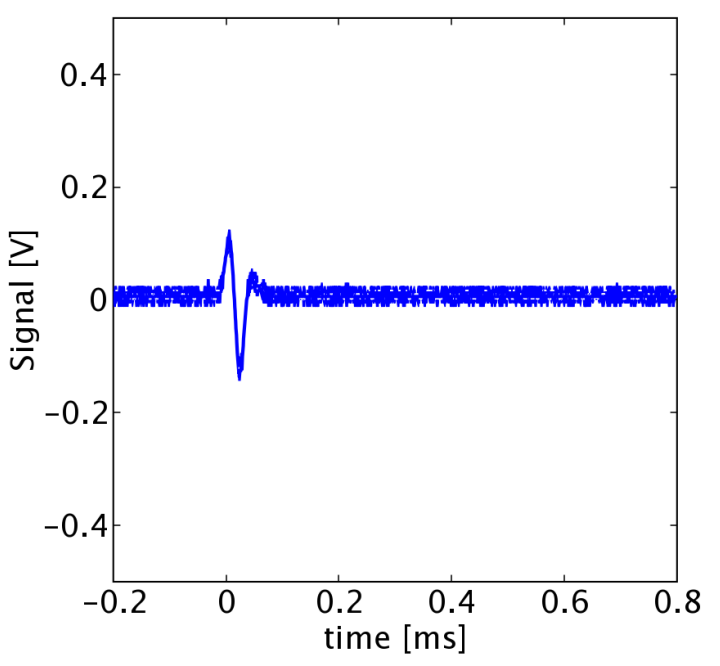

a) Signal from Magnetic Donut 1

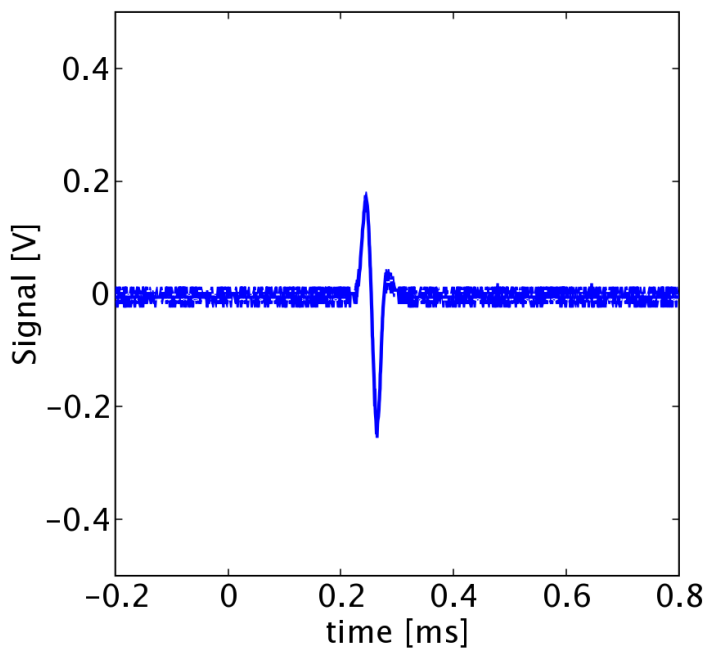

c) Signal from Magnetic Donut 3

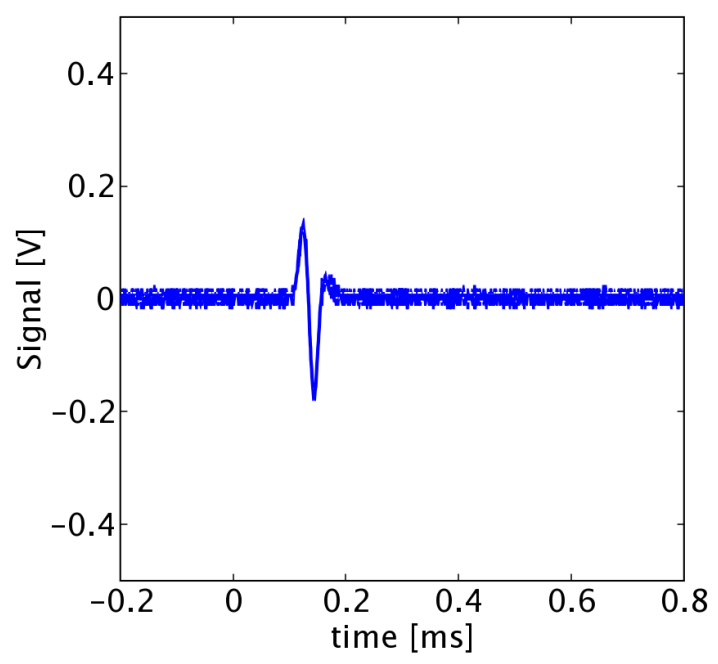

b) Signal from Magnetic Donut 2

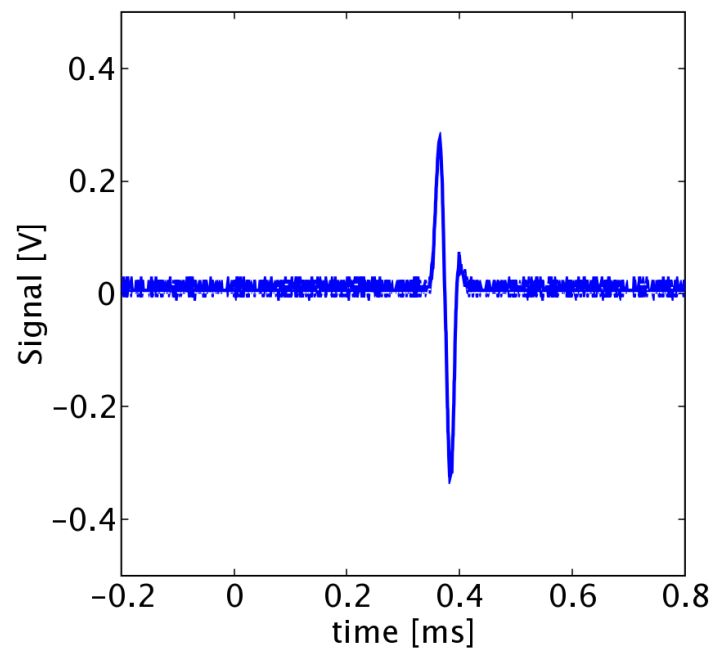

d) Signal from Magnetic Donut 4 
FIG. 37: Range Day 3, Shot 4

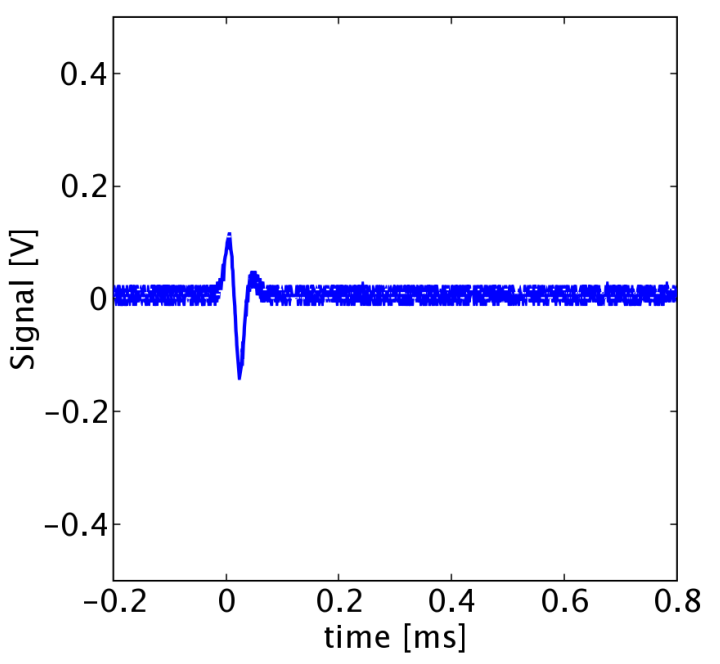

a) Signal from Magnetic Donut 1

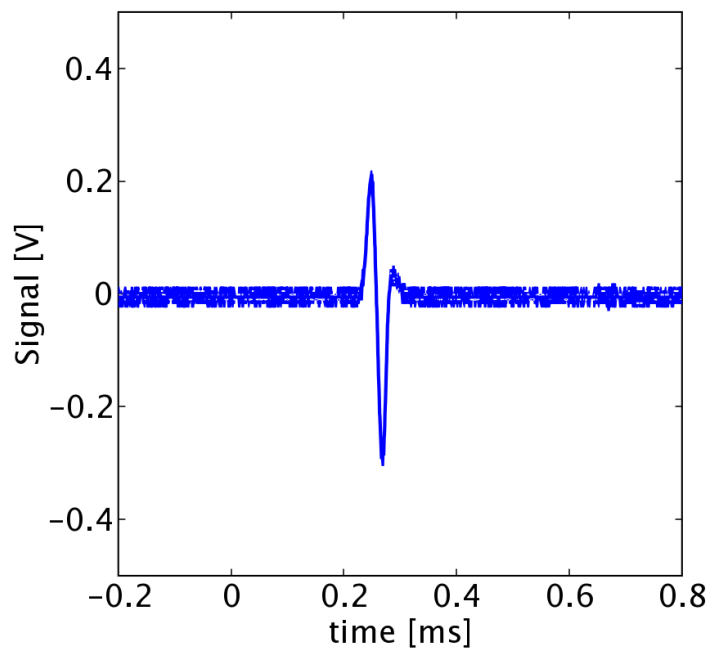

c) Signal from Magnetic Donut 3

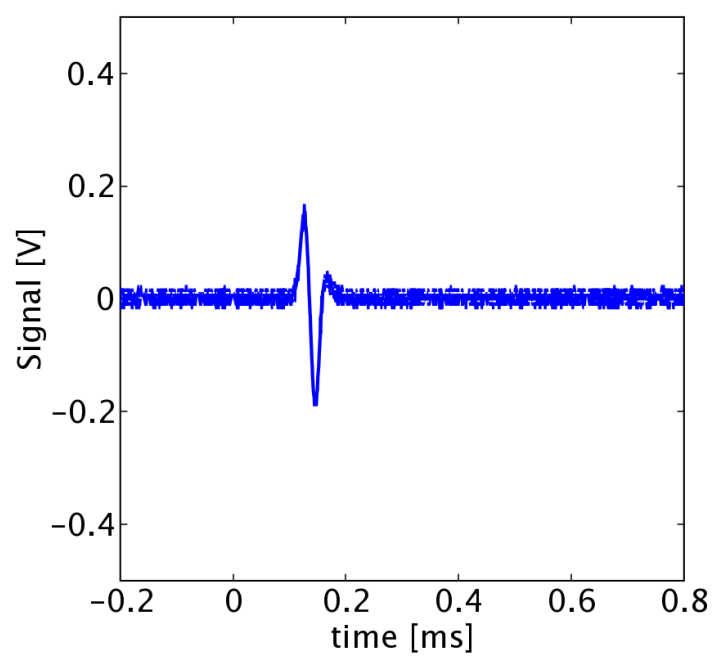

b) Signal from Magnetic Donut 2

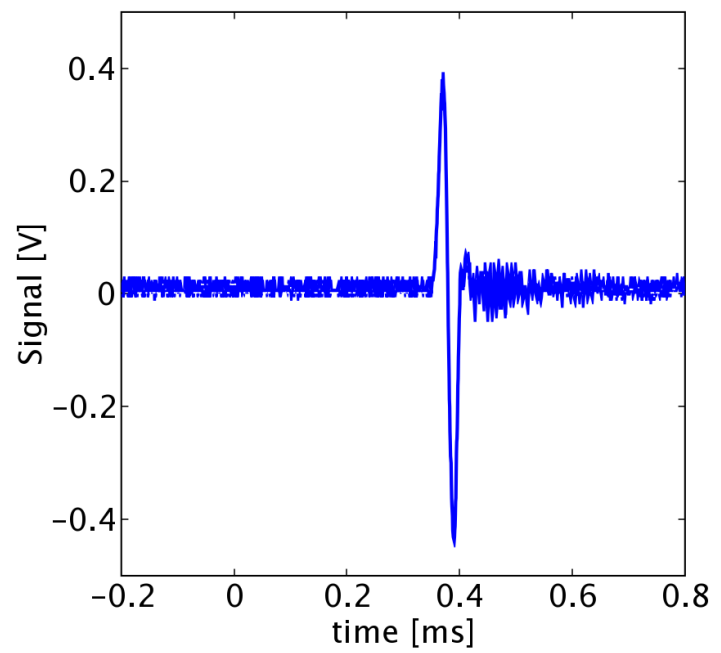

d) Signal from Magnetic Donut 4 
FIG. 38: Range Day 3, Shot 5
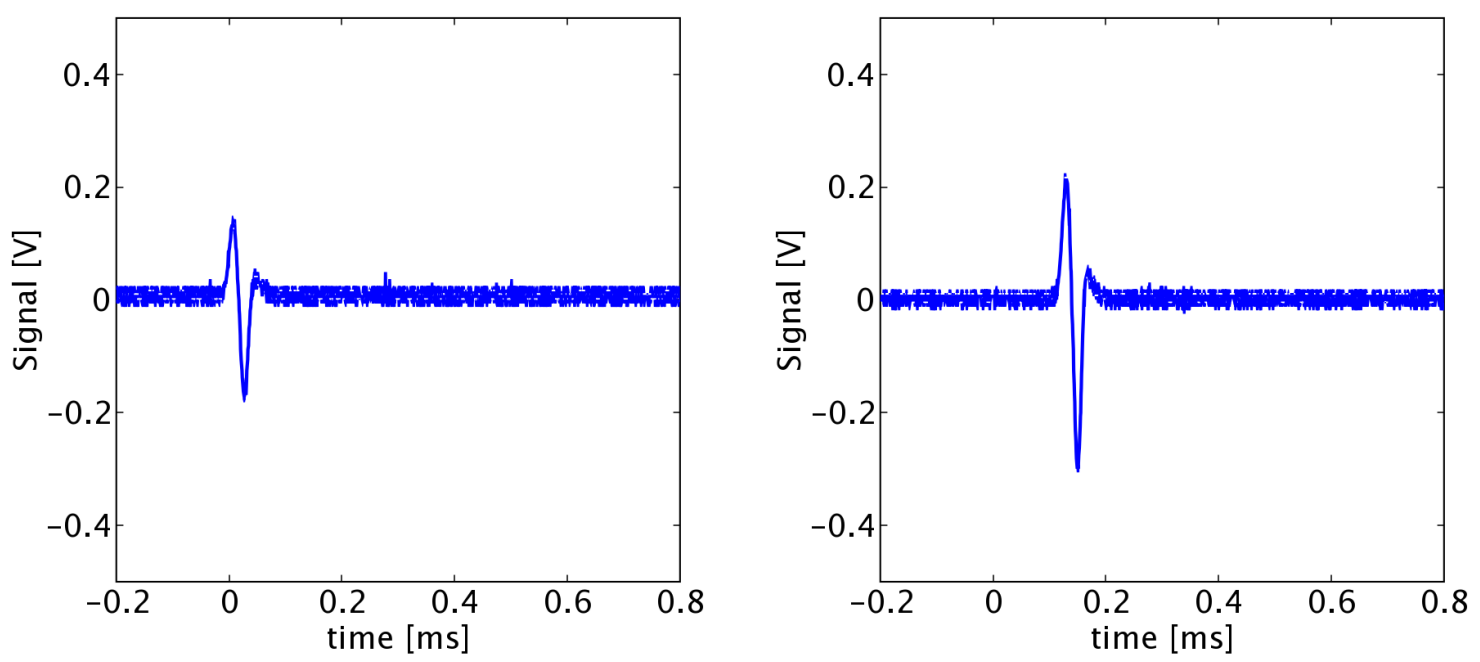

a) Signal from Magnetic Donut 1

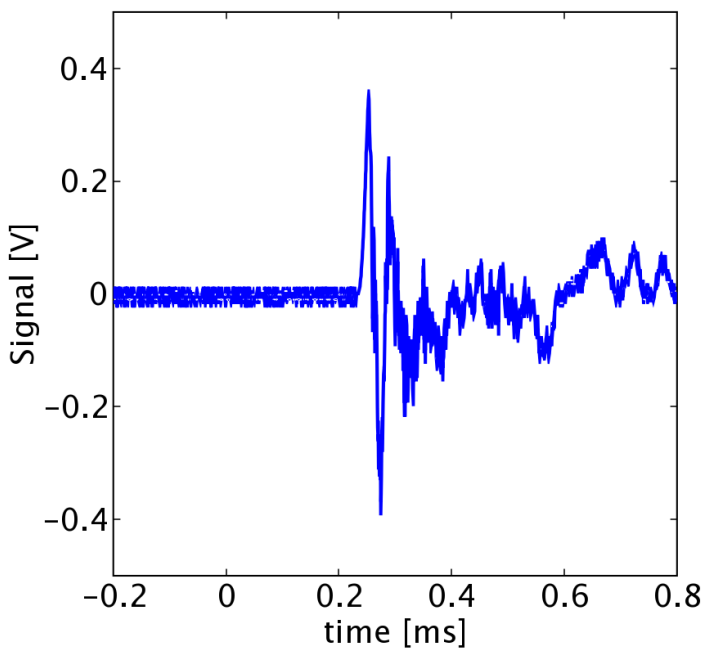

c) Signal from Magnetic Donut 3 b) Signal from Magnetic Donut 2

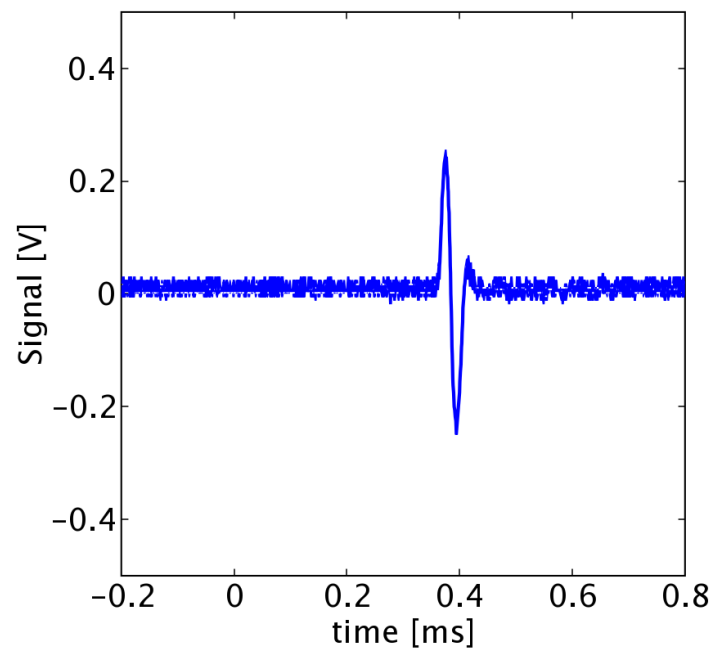

d) Signal from Magnetic Donut 4 
FIG. 39: Range Day 3, Shot 6

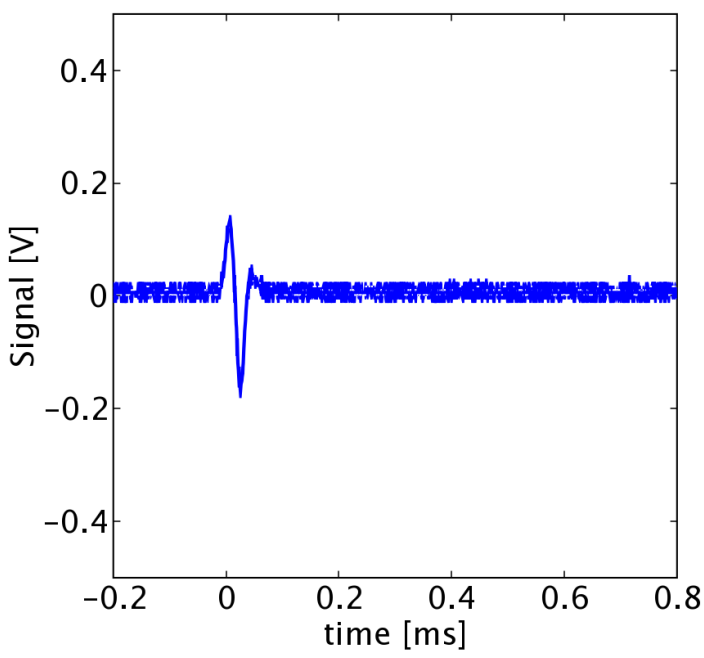

a) Signal from Magnetic Donut 1

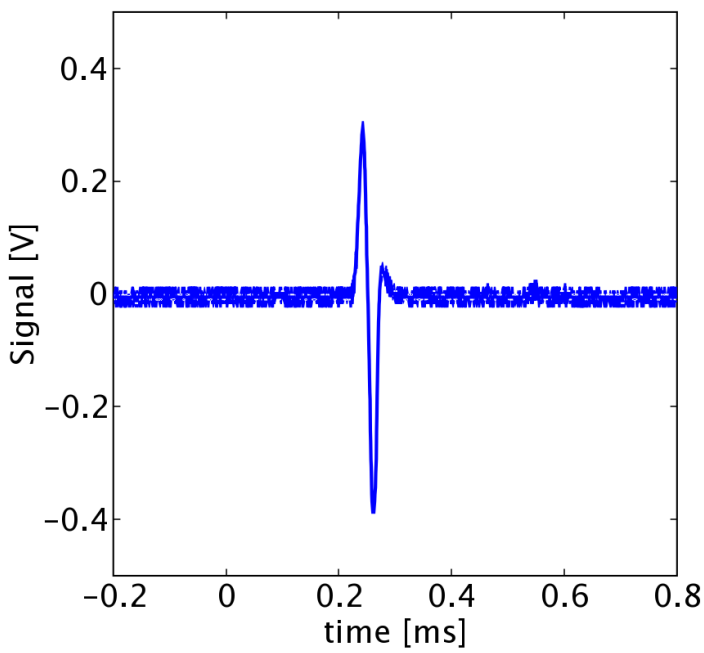

c) Signal from Magnetic Donut 3

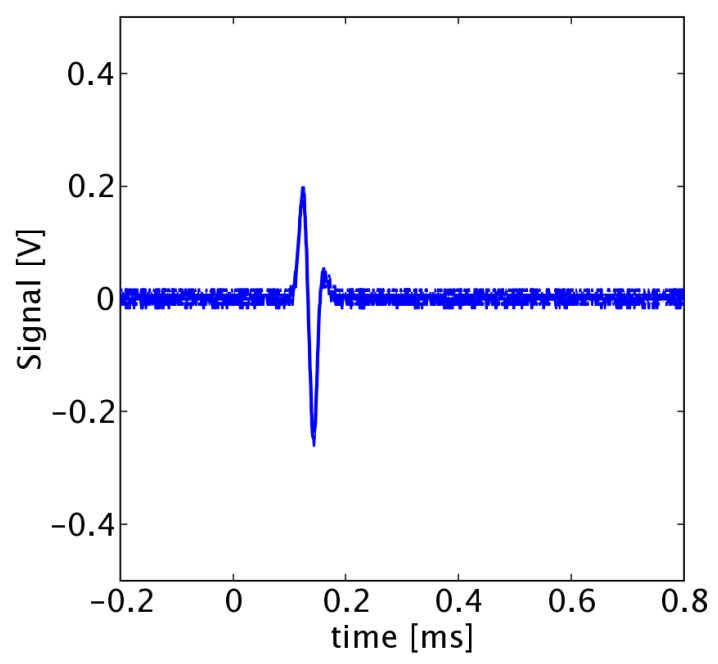

b) Signal from Magnetic Donut 2

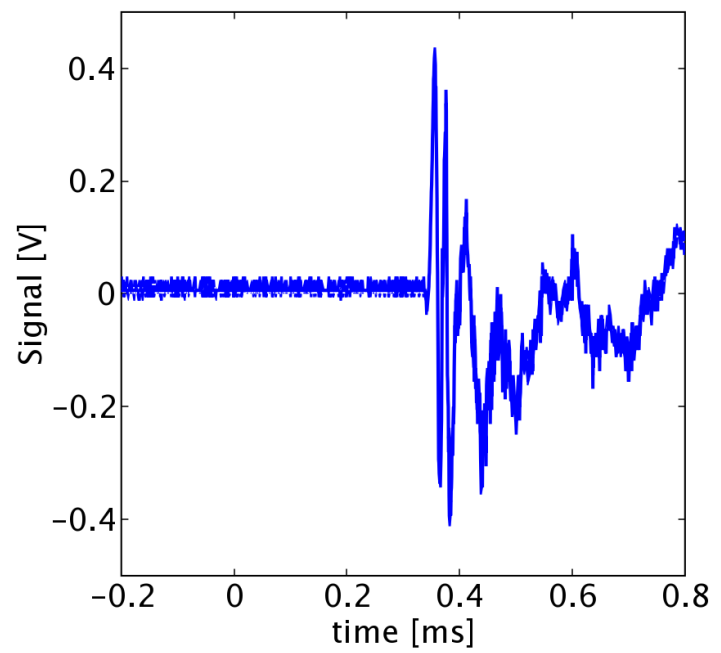

d) Signal from Magnetic Donut 4 
FIG. 40: Range Day 3, Shot 7

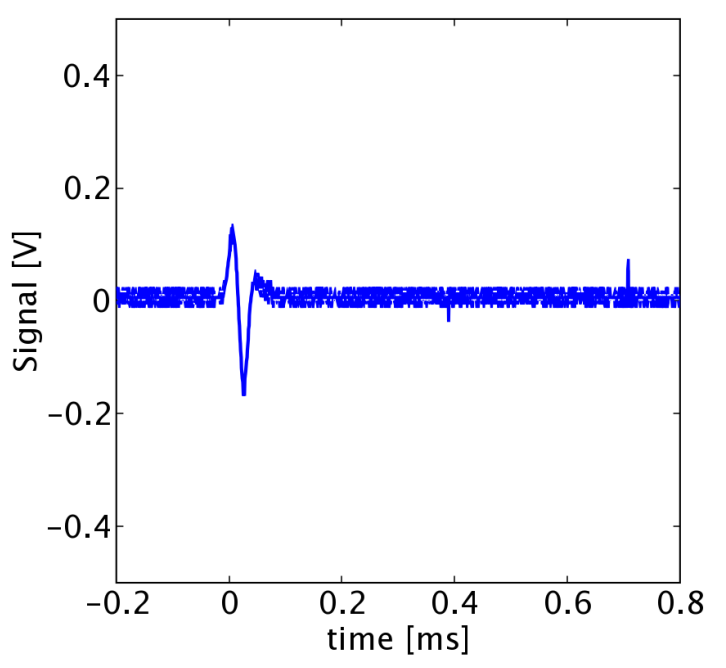

a) Signal from Magnetic Donut 1

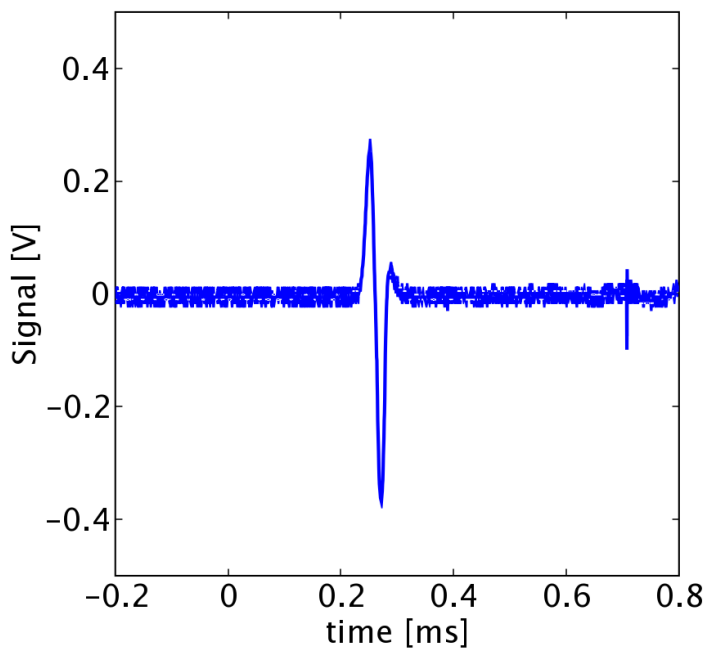

c) Signal from Magnetic Donut 3

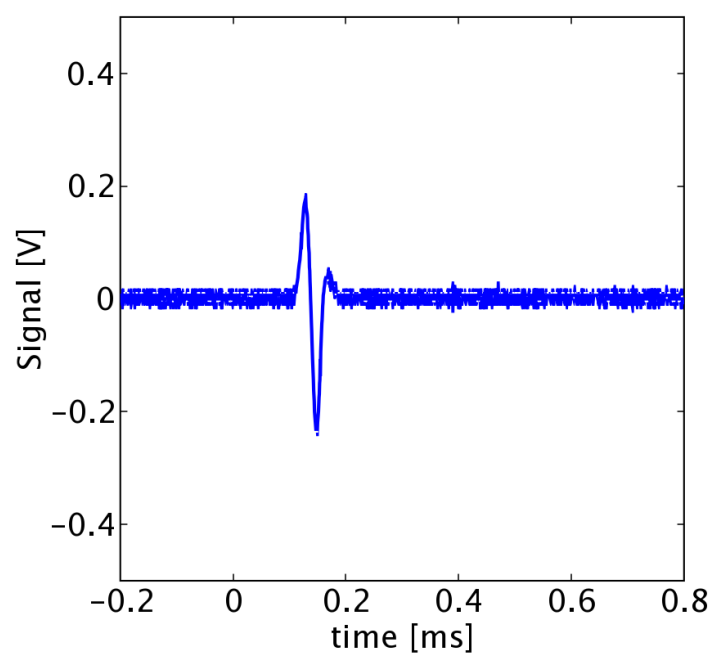

b) Signal from Magnetic Donut 2

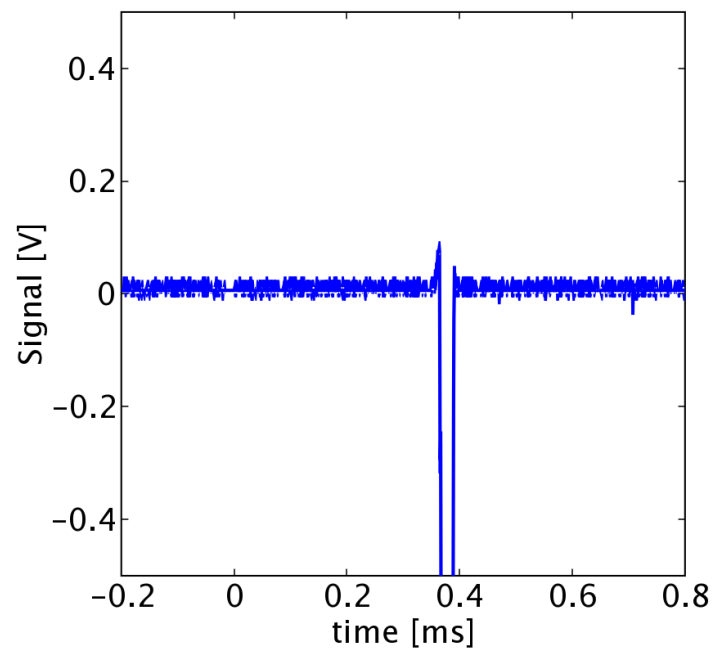

d) Signal from Magnetic Donut 4 
FIG. 41: Range Day 3, Shot 8
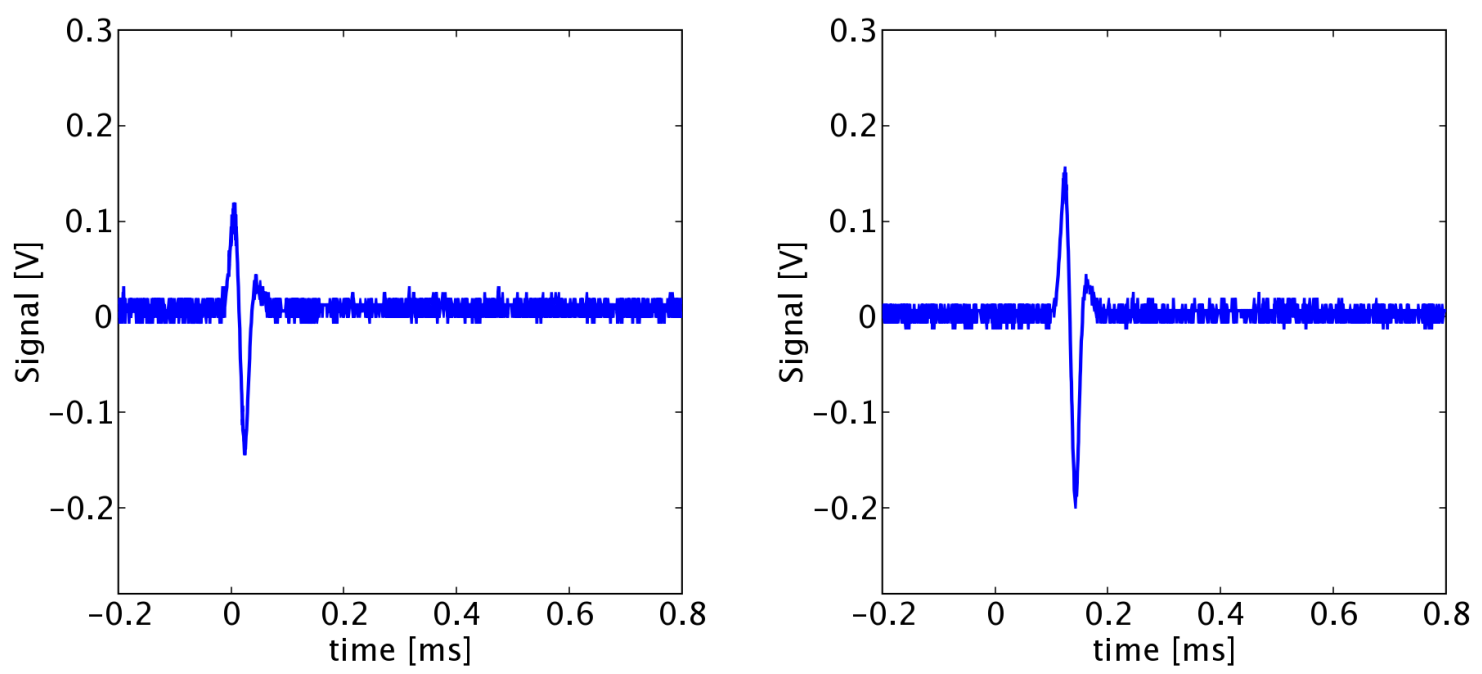

a) Signal from Magnetic Donut 1

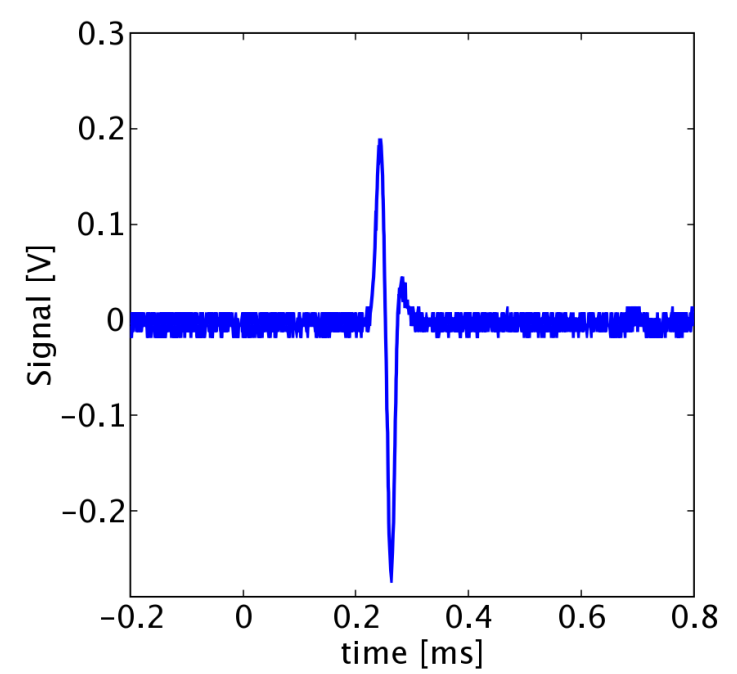

b) Signal from Magnetic Donut 2

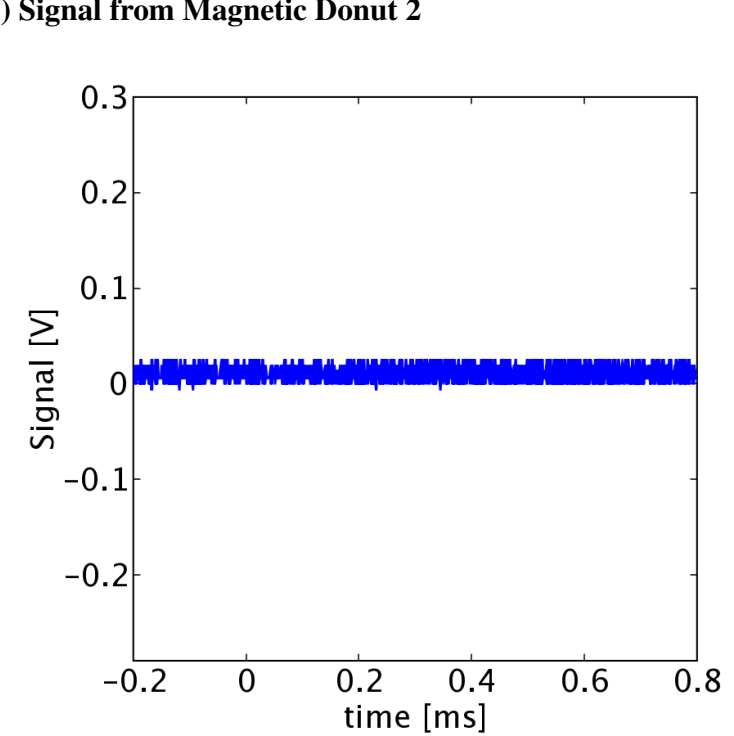

d) Signal from Magnetic Donut 4 c) Signal from Magnetic Donut 3 
FIG. 42: Range Day 3, Shot 9
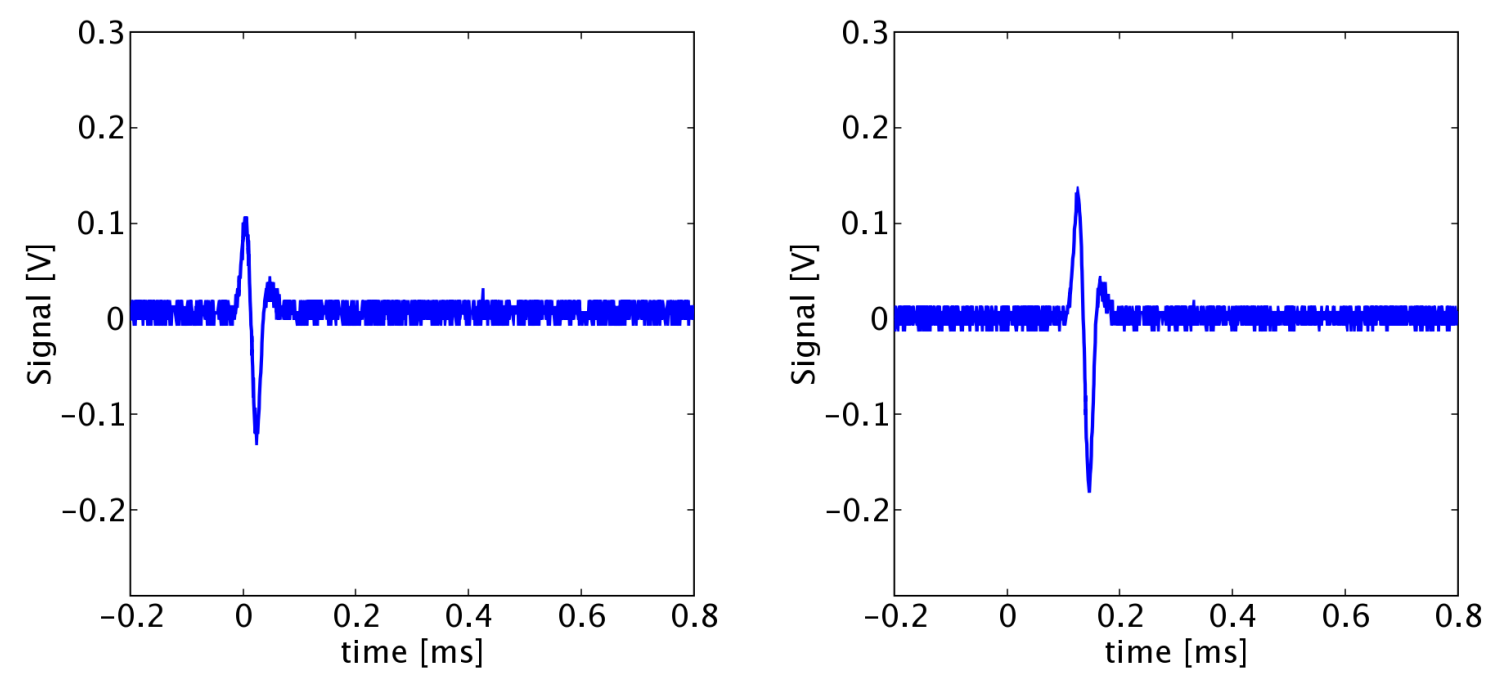

a) Signal from Magnetic Donut 1

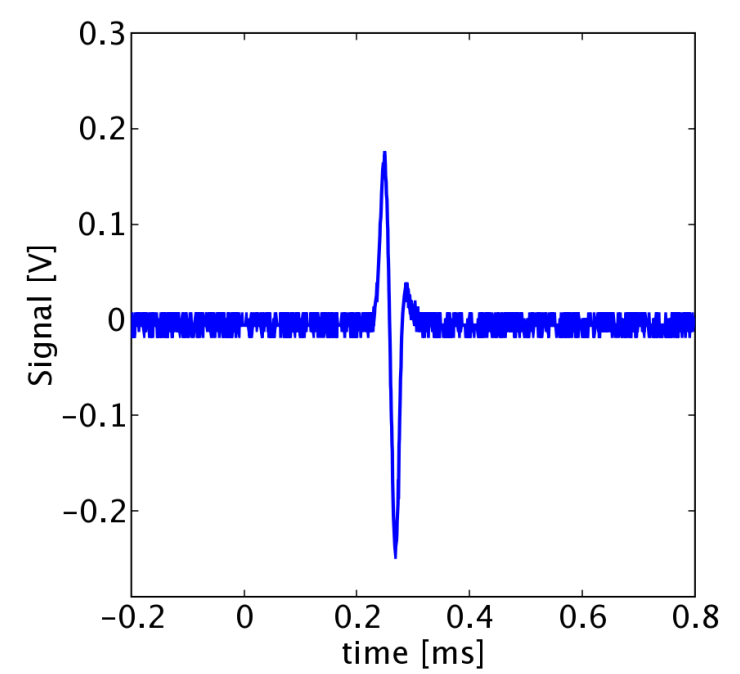

b) Signal from Magnetic Donut 2

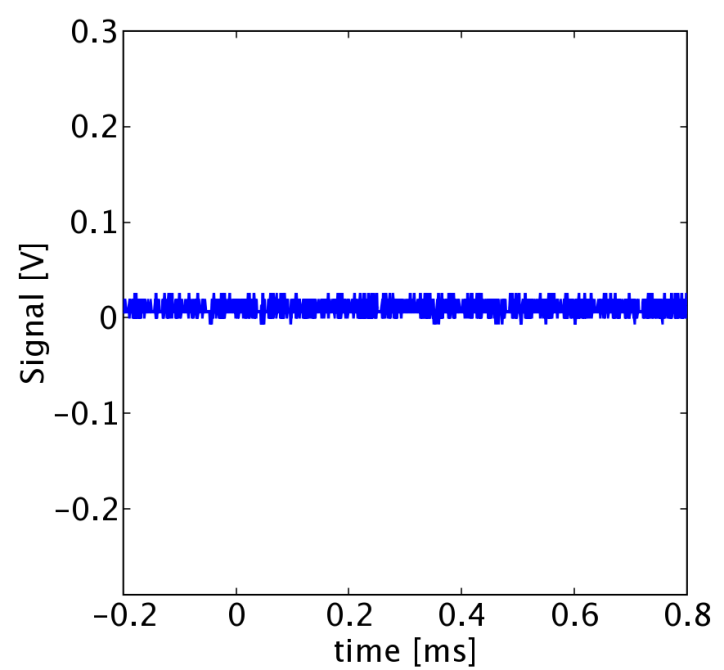

d) Signal from Magnetic Donut 4

c) Signal from Magnetic Donut 3

d) Signal from Magnetic Donut 4 
FIG. 43: Range Day 3, Shot 10
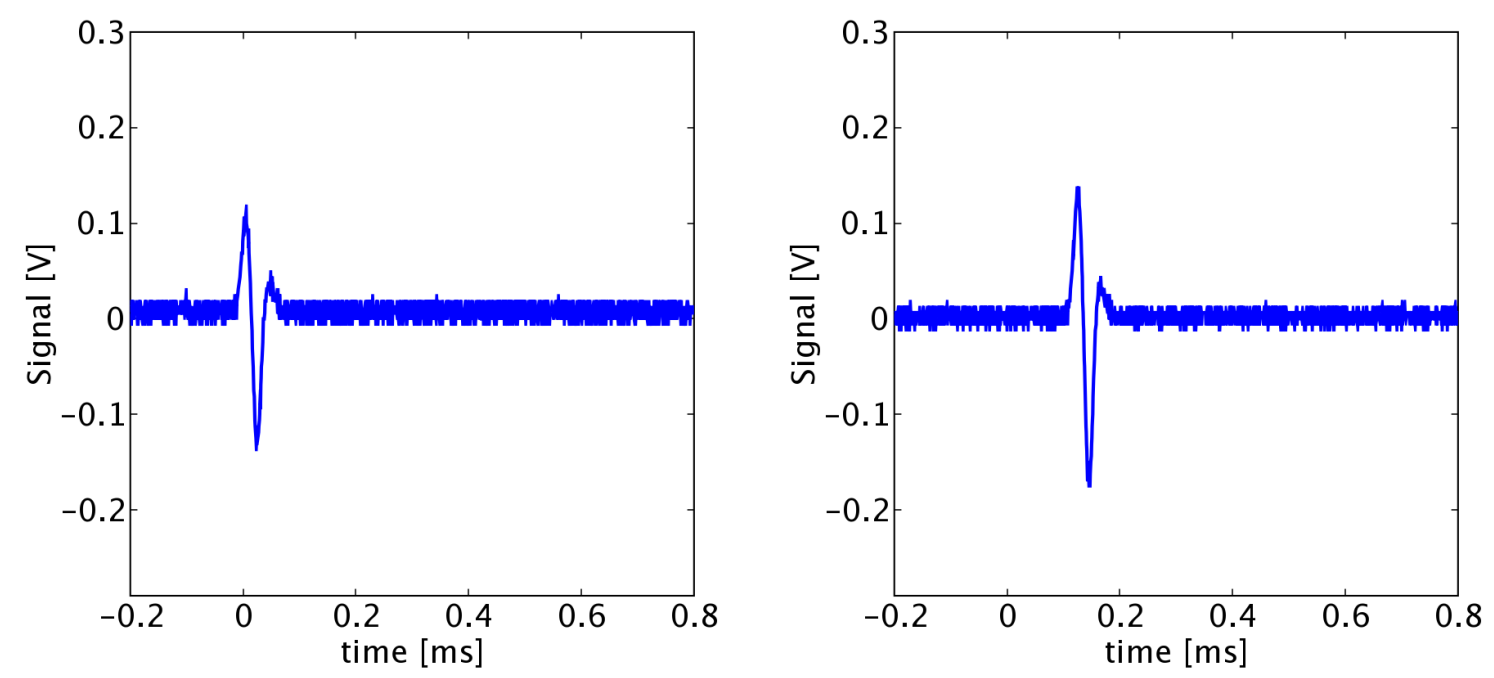

a) Signal from Magnetic Donut 1

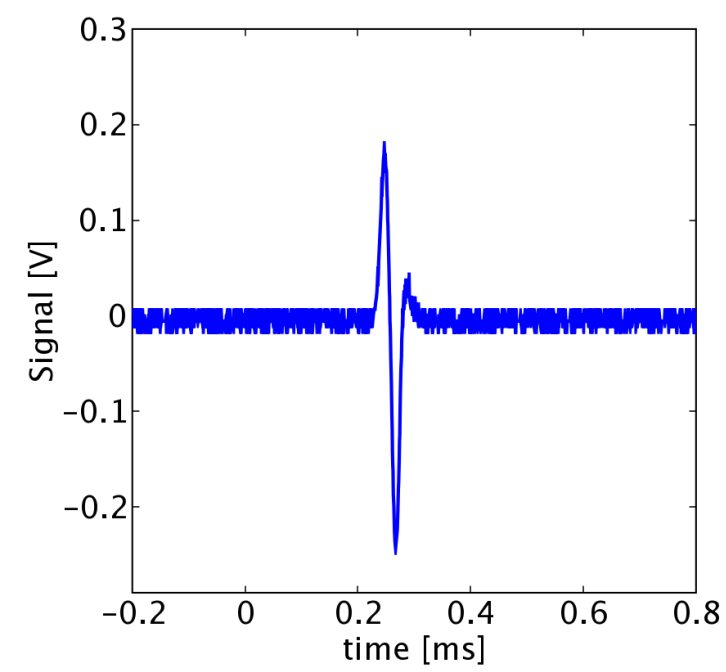

c) Signal from Magnetic Donut 3 b) Signal from Magnetic Donut 2

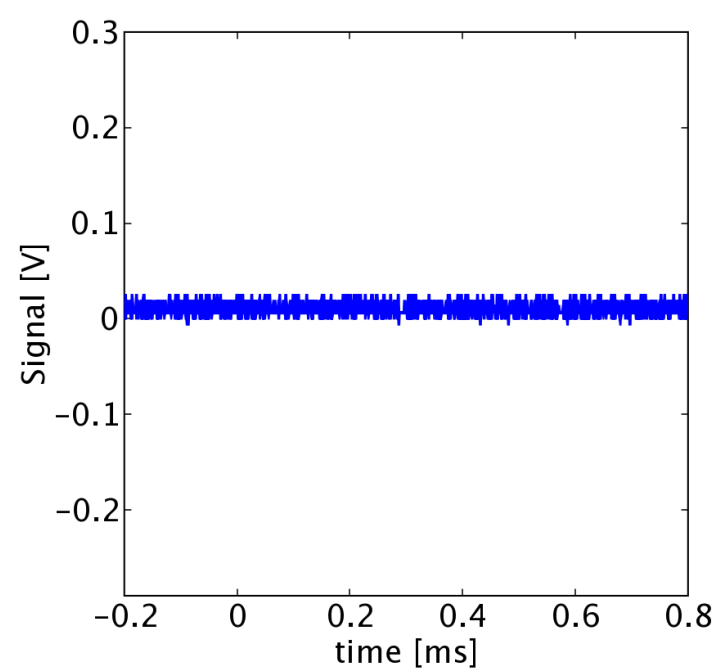

d) Signal from Magnetic Donut 4 
FIG. 44: Range Day 3, Shot 11
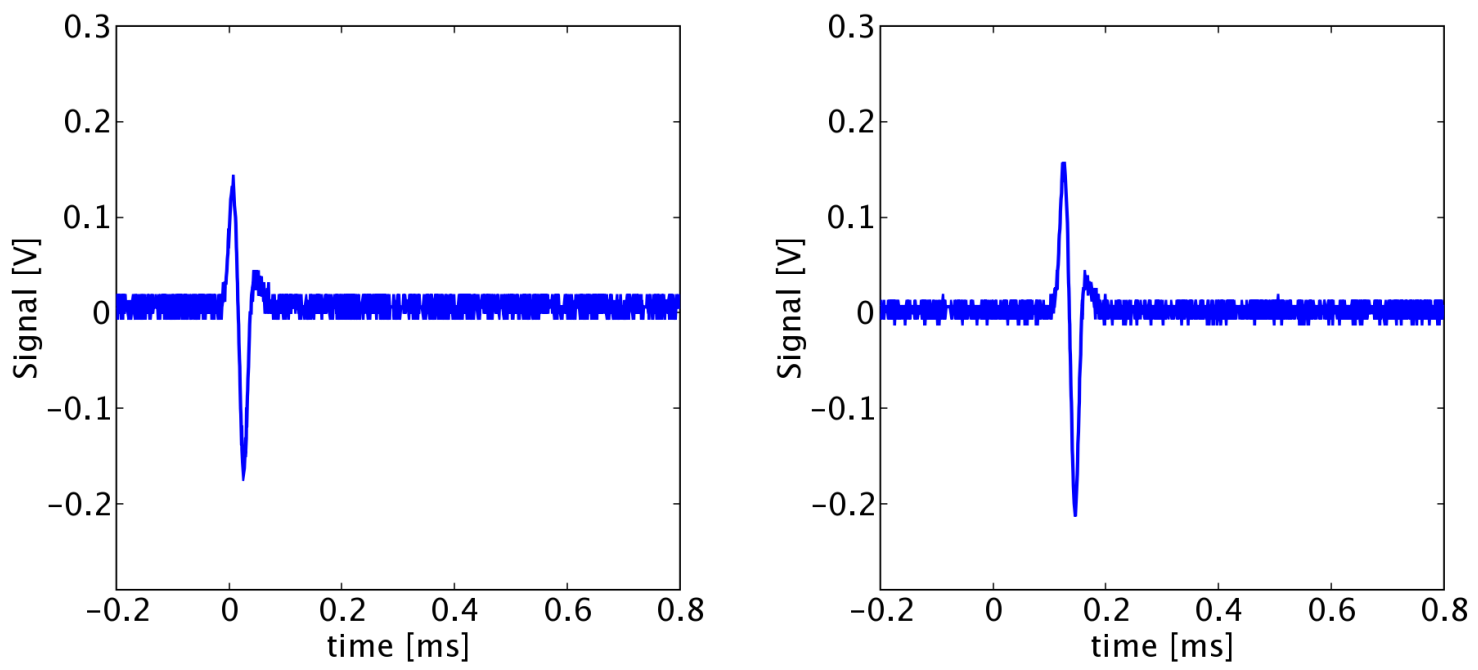

a) Signal from Magnetic Donut 1

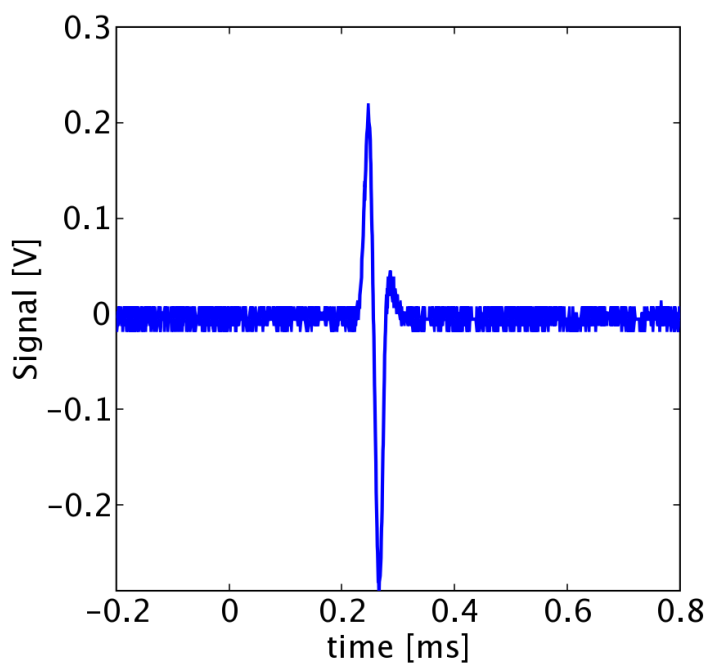

c) Signal from Magnetic Donut 3 b) Signal from Magnetic Donut 2

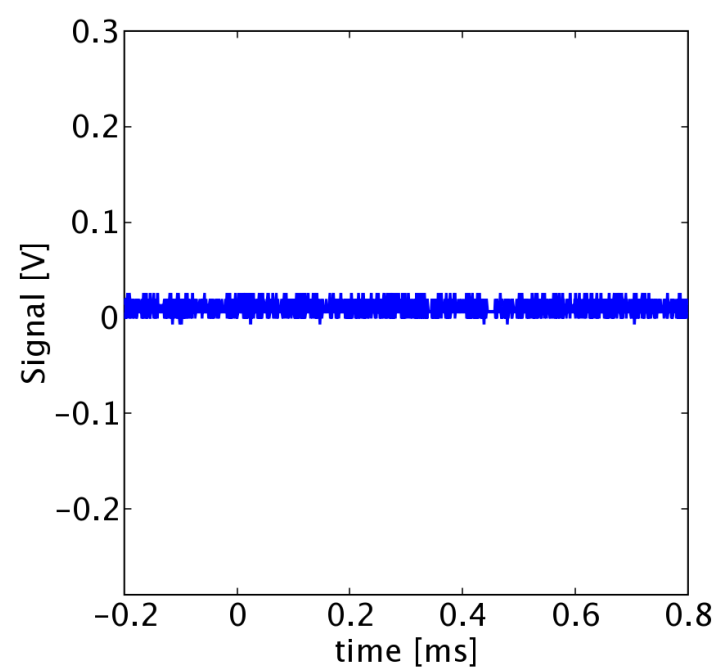

d) Signal from Magnetic Donut 4 
TABLE XI: Range day 3: Projectile position $x$ and time $t$ coordinates.

\begin{tabular}{|c|c|c|c|c|c|c|}
\hline donut & $x[\mathrm{~m}]$ & $t_{2}[\mathrm{~s}]$ & $t_{3}[\mathrm{~s}]$ & $t_{4}[\mathrm{~s}]$ & $t_{5}[\mathrm{~s}]$ & $t_{6}[\mathrm{~s}]$ \\
\hline 1 & 0.0000 & $1.7000 \mathrm{e}-05$ & $1.5375 \mathrm{e}-05$ & $1.5500 \mathrm{e}-05$ & $1.7917 \mathrm{e}-05$ & $1.7000 \mathrm{e}-05$ \\
\hline 2 & 0.1017 & $1.3850 \mathrm{e}-04$ & $1.3467 \mathrm{e}-04$ & $1.3612 \mathrm{e}-04$ & $1.4092 \mathrm{e}-04$ & $1.3375 \mathrm{e}-04$ \\
\hline 3 & 0.2032 & $2.6225 \mathrm{e}-04$ & $2.5517 \mathrm{e}-04$ & $2.5913 \mathrm{e}-04$ & $2.6355 e-04$ & $2.5255 \mathrm{e}-04$ \\
\hline 4 & 0.3043 & $3.7716 \mathrm{e}-04$ & $3.7463 \mathrm{e}-04$ & $3.7972 \mathrm{e}-04$ & $3.8446 \mathrm{e}-04$ & $3.7777 \mathrm{e}-04$ \\
\hline donut & $x[\mathrm{~m}]$ & $t_{7}[\mathrm{~s}]$ & $t_{8}[\mathrm{~s}]$ & $t_{9}[\mathrm{~s}]$ & $t_{10}[\mathrm{~s}]$ & $t_{11}[\mathrm{~s}]$ \\
\hline 1 & 0.0000 & $1.7083 \mathrm{e}-05$ & $1.5500 \mathrm{e}-05$ & $1.5000 \mathrm{e}-05$ & $1.5875 \mathrm{e}-05$ & $1.7083 \mathrm{e}-05$ \\
\hline 2 & 0.1017 & $1.3875 \mathrm{e}-04$ & $1.3342 \mathrm{e}-04$ & $1.3637 \mathrm{e}-04$ & $1.3625 \mathrm{e}-04$ & $1.3625 \mathrm{e}-04$ \\
\hline 3 & 0.2032 & $2.6225 \mathrm{e}-04$ & $2.5387 \mathrm{e}-04$ & $2.5933 \mathrm{e}-04$ & $2.5825 \mathrm{e}-04$ & $2.5731 \mathrm{e}-04$ \\
\hline 4 & 0.3043 & $0.0000 \mathrm{e}+00$ & $0.0000 \mathrm{e}+00$ & $0.0000 \mathrm{e}+00$ & $0.0000 \mathrm{e}+00$ & $0.0000 \mathrm{e}+00$ \\
\hline
\end{tabular}

TABLE XII: Range Day 3: Projectile velocities $v$.

\begin{tabular}{|c|c|c|c|c|c|c|}
\hline donut $\mathrm{A}$ & donut B & $v_{2}[\mathrm{~m} / \mathrm{s}]$ & $v_{3}[\mathrm{~m} / \mathrm{s}]$ & $v_{4}[\mathrm{~m} / \mathrm{s}]$ & $v_{5}[\mathrm{~m} / \mathrm{s}]$ & $v_{6}[\mathrm{~m} / \mathrm{s}]$ \\
\hline 1 & 2 & 836.8311 & 852.3224 & 842.9017 & 826.6263 & 870.8781 \\
\hline 1 & 3 & 828.6849 & 847.5481 & 834.2124 & 827.3798 & 862.8106 \\
\hline 1 & 4 & 844.9387 & 847.0702 & 835.5144 & 830.2195 & 843.4995 \\
\hline 2 & 3 & 820.6867 & 842.8217 & 825.6909 & 828.1356 & 854.8822 \\
\hline 2 & 4 & 849.0663 & 844.4591 & 831.8563 & 832.0342 & 830.4004 \\
\hline 3 & 4 & 879.6302 & 846.1109 & 838.1448 & 835.9886 & 807.1739 \\
\hline donut $\mathrm{A}$ & donut B & $v_{7}[\mathrm{~m} / \mathrm{s}]$ & $v_{8}[\mathrm{~m} / \mathrm{s}]$ & $v_{9}[\mathrm{~m} / \mathrm{s}]$ & $v_{10}[\mathrm{~m} / \mathrm{s}]$ & $v_{11}[\mathrm{~m} / \mathrm{s}]$ \\
\hline 1 & 2 & 835.6849 & 862.2614 & 837.6927 & 844.6522 & 853.2168 \\
\hline 1 & 3 & 828.9666 & 852.5853 & 831.7937 & 838.5147 & 846.0047 \\
\hline 1 & 4 & 0.0000 & 0.0000 & 0.0000 & 0.0000 & 0.0000 \\
\hline 2 & 3 & 822.3481 & 843.1134 & 825.9707 & 832.4590 & 838.9056 \\
\hline 2 & 4 & 0.0000 & 0.0000 & 0.0000 & 0.0000 & 0.0000 \\
\hline 3 & 4 & 0.0000 & 0.0000 & 0.0000 & 0.0000 & 0.0000 \\
\hline
\end{tabular}

